\title{
Equivalence of Finite-Valued Tree Transducers Is Decidable
}

\author{
H. Seidl \\ Fachbereich Informatik, Universität des Saarlandes, \\ Postfach 151150, D-66041 Saarbrücken, Germany
}

\begin{abstract}
A bottom-up finite state tree transducer (FST) $M$ is called $k$-valued iff for every input tree there are at most $k$ different output trees. $M$ is called finite-valued iff it is $k$-valued for some $k$. We show that it is decidable for every $k$ whether or not a given FST $M$ is $k$-valued. We give an effective characterization of all finite-valued FSTs and derive a (sharp) upper bound for the valuedness provided it is finite. We decompose a finite-valued FST $M$ into a finite number of single-valued FSTs. This enables us to prove: it is decidable whether or not the translation of an FST $M$ is included in the translation of a finite-valued FST $M^{\prime}$. We also consider these questions for size-valuedness.
\end{abstract}

\section{Introduction}

A bottom-up finite state tree transducer (here FST for short) is a finite state device which produces its output tree while consuming a given input tree in a bottom-up fashion. Since multiple occurrences of variables in patterns are allowed an FST is able to generate several identical copies of images of subtrees. Since some variables can be missing, the image of a correctly parsed subtree may be dropped again.

In compiler construction finite state transducers are an important tool for manipulating abstract syntax trees. For example, consider the code generation phase. The different possible target code sequences for an abstract syntax tree $t$ correspond to the different outputs $s$ possibly produced by an FST [GS2]. A good survey on tree automata theory and its applications may be found in [GS1].

Formally, FSTs can be viewed as one possible generalization of nondeterministic generalized sequential machines (NGSMs). Therefore, the natural question arises whether or not results about NGSMs can be extended to FSTs. In this paper we investigate finite-valuedness and the equivalence problem for FSTs. 
Two FSTs are called equivalent iff they define the same translation. It is well known that the equivalence problem for NGSMs is undecidable. However, in 1986 Culik II and Karhumäki proved that the equivalence problem is decidable at least for finite-valued NGSMs [CK]. They employed the (confirmed) Ehrenfeucht conjecture for proving their procedure to be recursive; therefore, no estimation of its complexity is known. In 1988 Weber came up with a totally different decision procedure (running in double exponential time) which was based on a careful structural analysis of finite-valued NGSMs [W2]. In 1989 Weber showed that the equivalence problem was decidable even for NGSMs having finite length-degree [W3].

Knowledge about finite-valuedness and the equivalence problem for finite tree transducers is comparatively poor. In 1978 Zachar showed that equivalence was decidable for deterministic FSTs [Z]. In 1980 Engelfriet exhibited a nice generalization (T1) of a word lemma by Schützenberger [Sc] to trees which allows the decision whether or not a given FST is single-valued [En]. Note that any algorithm which decides single-valuedness can be used to decide equivalence of single-valued FSTs. For the sake of completeness we also mention the (stronger) result of Esik [Es] which showed that single-valuedness is decidable for top-down FSTs as well. Also, as a special case of this Courcelle and Franchi-Zannettacchi exhibited an interesting method to decide whether or not two deterministic top-down transducers are equivalent [CF].

We proceed in the spirit of [W4], [W2], and [En]. In fact, it turns out that most of the proofs deal with sizes of output trees (thus generalizing the notion of "length" for words). Therefore, in parallel with a theory of finite-valued FSTs, we develop a theory of FSTs where the sets of output sizes have bounded cardinalities.

We start with an investigation of the combinatorics of trees. Employing Ramsey's theory we generalize Engelfriet's property (T1) (Theorem 1.5). This enables us to prove: for every $k$ it is decidable in nondeterministic polynomial time whether a given FST is not $k$-valued (Theorem 2.2). Moreover, we consider polynomials with rational coefficients of degree at most 1 . Surprisingly, they have similar cancellation and factorization properties as trees. Therefore, analogous considerations allow us to prove: for every $k$ it is decidable in polynomial space whether or not an FST is $k$-size-valued (Theorem 2.3).

In Section 3 we exhibit two necessary conditions (F1) and (F2) for an FST to be finite-valued. At least two-thirds of the proof consists in proving that the size versions (S1) and (S2) of (F1) and (F2) are necessary conditions for an FST to be finite size-valued.

In Section 4 we prove that the necessary conditions (F1) and (F2) for finite-valuedness ((S1) and (S2) for finite size-valuedness) are decidable (Theorem 4.1). This is done by proving that they only have to be verified for input trees up to a fixed depth.

In Section 5 we prove that the necessary conditions (F1) and (F2) ((S1) and (S2)) of Section 3 are also sufficient to derive an upper bound for the valuedness of an FST (size-valuedness of an FST). Property (F1) subsumes the criteria for NGSMs to be finite-valued given in [WS], [W1], and [W4]. Due to the more 
complicated structure of trees this generalization is by no means obvious. Property (F2) has no analogue for NGSMs. From the characterization of finite-valuedness we obtain the following results (Theorems 5.4, 5.5, and 5.7):

(1) It can be decided in nondeterministic polynomial time whether an FST $M$ is infinite-valued.

(2) If $M$ is finite-valued, then its valuedness is bounded by $2^{2^{P(|M|)}}$ for some polynomial $P$ independent of $M$.

The upper bound in (2) is sharp in the following sense: there are finite-valued FSTs $M^{\prime}$ of arbitrarily large size such that their valuedness is at least $2^{2^{P\left(M^{\prime}\right)}}$ for some polynomial $P^{\prime}$.

Corresponding results also hold for size-valuedness. Here we find:

(1') It can be decided in polynomial space whether or not an FST $M$ is finite size-valued.

(2') If $M$ is finite size-valued, then its size-valuedness is bounded by $2^{2 c \cdot|M| \cdot \log (L+1)}$ for some constant $c>0$ independent of $M(L$ denotes the maximal number of successors of a node of an input tree).

Finally, Section 6 shows that the ideas of [W2] for NGSMs can be extended to FSTs. We employ the precise knowledge about the structure of finite-valued FSTs of Section 5 to decompose a finite-valued FST into a finite number of single-valued FSTs (Theorem 6.2). The decomposition is based on a classification of accepting computations such that the following holds: if two accepting computations for the same input tree $t$ admit the same specification $\gamma$, then they produce the same output. Secondly, we observe that an FST can decide "on-line" whether or not a simulated accepting computation admits some specification $\gamma$. By employing again our generalization of Engelfriet's property (T1) we finally derive the following result (Theorem 6.4): It can be decided for an FST $M$ and a finite-valued FST $M^{\prime}$ in nondeterministic time $2^{2^{2 \operatorname{poly}\left(M(M)+\mid M^{\prime}\right)}}$ whether the translation of $M$ is not included in the translation of $M^{\prime}$. Again, a corresponding result for size-valuedness holds as well. We conclude with open problems.

\section{Trees and Polynomials}

In this section we give basic definitions and state some fundamental properties about trees. Especially, we generalize Engelfriet's property (T1) of [En] into two directions. For this we employ a proposition taken from Ramsey Theory which is presented in the Appendix. One generalization of Engelfriet's property (T1) enables us to decide whether or not a given FST is $k$-valued; the second generalization is used in Section 6 to show that it can be decided whether or not the translation defined by an FST is included in the union of the translations of a finite number of single-valued FSTs.

In a second subsection we consider the same questions for polynomials of degree at most 1 . 
A ranked alphabet or signature is a pair $(\Sigma, \rho)$, where $\Sigma$ is a finite alphabet and $\rho: \Sigma \rightarrow \mathbb{N}_{0}$ is a function mapping symbols to their rank ( $\mathbb{N}$ denotes the set of positive integers and $\mathbb{N}_{0}$ the set of nonnegative integers). Usually, if $\rho$ is understood we write $\Sigma$ for short and define $\Sigma_{j}:=\rho^{-1}(j)$. The maximal $j$ such that $\Sigma_{j} \neq \varnothing$ is also called the rank of $\Sigma$. $T_{\Sigma}$ denotes the free $\Sigma$-algebra of (finite ordered $\Sigma$-labeled) trees, i.e., $T_{\Sigma}$ is the smallest set $T$ satisfying (i) $\Sigma_{0} \subseteq T$ and (ii) if $a \in \Sigma_{m}$ and $t_{1}, \ldots, t_{m} \in T$, then $a\left(t_{1}, \ldots, t_{m}\right) \in T$. Note: (i) can be viewed as the subcase of (ii) where $m=0$.

The depth of a tree $t \in T_{\Sigma}$, depth $(t)$, is defined by depth $(t):=0$ if $t \in \Sigma_{0}$, and $\operatorname{depth}(t):=1+\max \left\{\operatorname{depth}\left(t_{1}\right), \ldots, \operatorname{depth}\left(t_{m}\right)\right\}$ if $t=a\left(t_{1}, \ldots, t_{m}\right)$ for $m>0$.

The set of nodes of (or occurrences in) $t, O(t)$ is the subset of $\mathbb{N}^{*}$ defined by

$$
O(t):=\{\varepsilon\} \cup \bigcup_{j=1}^{m} j \cdot O\left(t_{j}\right) \quad \text { where } t=a\left(t_{1}, \ldots, t_{m}\right) \text { for } m \geq 0 ;
$$

especially, the empty string $\varepsilon$ is the root of $t$.

$O(t)$ is partially ordered by the prefix relation and totally ordered with respect to the lexicographical ordering.

$t$ defines maps $t\left({ }_{-}\right): O(t) \rightarrow \Sigma$ and $t /_{-}: O(t) \rightarrow T_{\Sigma}$ mapping each node $o$ of $t$ to its label or the subtree of $t$ with root $o$, respectively. We have, for $t=a\left(t_{1}, \ldots, t_{m}\right)$,

$$
t(o):=\left\{\begin{array}{ll}
a & \text { if } \quad o=\varepsilon, \\
t_{j}\left(o^{\prime}\right) & \text { if } \quad o=j \cdot o^{\prime},
\end{array} \quad \text { and } \quad t / o:=\left\{\begin{array}{lll}
t & \text { if } \quad o=\varepsilon, \\
t_{j} / o^{\prime} & \text { if } \quad o=j \cdot o^{\prime} .
\end{array}\right.\right.
$$

For every subset $N$ of $\Sigma$ define the $N$-size $|t|_{N}$ of $t=a\left(t_{1}, \ldots, t_{m}\right)$ by $|t|_{N}:=$ $1+\sum_{j=1}^{m}\left|t_{j}\right|_{N}$ if $a \in N$ and $|t|_{N}=\sum_{j=1}^{m}\left|t_{j}\right|_{N}$ if $a \notin N$. If $N=\Sigma$, we drop the index.

Let $X$ denote a set of variables of rank 0 . Then define $T_{\Sigma}(X):=T_{\Sigma \cup X}$. (Clearly, $T_{\Sigma} \subseteq T_{\Sigma}(X)$.) We use this different notation in order to indicate those variables which are to be substituted. $t \in T_{\Sigma}(X)$ is called $X$-proper iff every $x \in X$ occurs in $t$ exactly once. If $X$ is understood, we call $t$ simply proper.

Every map $\theta: X \rightarrow T_{\Sigma}(X)$ can be extended to a map $\theta: T_{\Sigma}(X) \rightarrow T_{\Sigma}(X)$. For $t \in T_{\Sigma}(X), t \theta$ is obtained from $t$ by replacing all the occurrences of variables $x \in X$ with $x \theta, \theta$ is called an $X$-substitution or just substitution if $X$ is understood. We write $\theta$ to the right of the argument in accordance with the prefix notation of trees. If $X=\left\{x_{1}, \ldots, x_{m}\right\}$ and $x_{i} \theta=t_{i}$, we denote $t \theta$ by $t\left[t_{1}, \ldots, t_{m}\right]$. We also write $t t_{1}$ instead of $t\left[t_{1}\right]$.

The set $T_{\Sigma}\left(x_{1}\right):=T_{\Sigma}\left(\left\{x_{1}\right\}\right)$ is a monoid with respect to substitution (the neutral element is $\left.x_{1}\right)$. $T_{\Sigma}\left(x_{1}\right)$ is not a free monoid. Especially, $t_{1} t_{2}=t_{1}$ if $t_{1}$ does not contain an occurrence of $x_{1}$.

Propositions 1.1-1.4 (mainly taken from [En]) state basic properties of $T_{\Sigma}\left(x_{1}\right)$.

Proposition 1.1. Assume $s_{1}, s_{2}, t_{1}, t_{2}, t_{1}^{\prime}, t_{2}^{\prime} \in T_{\Sigma}\left(x_{1}\right)$.

(i) Bottom Cancellation. Assume $t_{1} \neq t_{1}^{\prime}$. Then $s_{1} t_{1}=s_{2} t_{1}$ and $s_{1} t_{1}^{\prime}=s_{2} t_{1}^{\prime}$ implies $s_{1}=s_{2}$.

(ii) Top Cancellation. Assume $x_{1}$ occurs in $s_{1}$. Then $s_{1} t_{1}=s_{1} t_{2}$ implies $t_{1}=t_{2}$.

(iii) Factorization. Assume $t_{i} \neq t_{i}^{\prime}$ for $i=1,2$. Then $s_{1} t_{1}=s_{2} t_{2}$ and $s_{1} t_{1}^{\prime}=s_{2} t_{2}^{\prime}$ implies $s_{1} r=s_{2}$ or $s_{1}=s_{2} r$ for some $r \in T_{\Sigma}\left(x_{1}\right)$. 
An immediate consequence of assertion (iii) is:

(iv) Assume $t_{i} \neq t_{i}^{\prime}$ for $i=1,2$ and $\left|s_{1}\right|_{\Sigma}=\left|s_{2}\right|_{\Sigma}$. Then $s_{1} t_{1}=s_{2} t_{2}$ and $s_{1} t_{1}^{\prime}=s_{2} t_{2}^{\prime}$ implies $s_{1}=s_{2}$.

Proof of (iii). First we define a relation $\leq \subseteq T_{\Sigma}\left(x_{1}\right) \times T_{\Sigma}\left(x_{1}\right)$. For $s, t \in T_{\Sigma}\left(x_{1}\right), s \leq t$ iff $O(s) \subseteq O(t)$ and, $\forall o \in O(s), s(o) \neq x_{1}$ implies $s(o)=t(o)$. Intuitively this means that $s$ is a "prefix" of $t$, i.e., $s$ is obtained from $t$ by changing certain subtrees (not necessarily all the same) into $x_{1}$. Thus, $t=s r$ implies $s \leq t$ but not vice versa. We prove the following fact:

(*) Assume $s_{1}, s_{2}, t \in T_{\Sigma}\left(x_{1}\right)$ where $s_{1} \leq t$ and $s_{2} \leq t$. If not $s_{1} \leq s_{2}$, then $\exists o \in O\left(s_{1}\right) \cap O\left(s_{2}\right), s_{1}(o) \neq x_{1}$, and $s_{2}(o)=x_{1}$.

Assume

$$
s_{2}(o)=x_{1} \quad \text { implies } \quad s_{1}(o)=x_{1}, \quad \forall o \in O\left(s_{1}\right) \cap O\left(s_{2}\right) .
$$

In order to prove fact $(*)$ it suffices to deduce from (1) that $s_{1} \leq s_{2}$. Since $s_{i} \leq t$ for $i=1,2$ we have

$$
\begin{aligned}
& s_{1}(o) \neq x_{1} \text { and } s_{2}(o) \neq x_{1} \text { implies } s_{1}(o)=s_{2}(o), \\
& \forall o \in O\left(s_{1}\right) \cap O\left(s_{2}\right) .
\end{aligned}
$$

From (1) and (2) it follows that $s_{1} \leq s_{2}$ already holds provided $O\left(s_{1}\right) \subseteq O\left(s_{2}\right)$ (and hence $\left.O\left(s_{1}\right)=O\left(s_{1}\right) \cap O\left(s_{2}\right)\right)$. To prove that $O\left(s_{1}\right) \subseteq O\left(s_{2}\right)$ we assume for a contradiction that $s_{1}$ contains a node $o \notin O\left(s_{2}\right)$. Then $o=o_{2} o^{\prime}$ where $o_{2}$ is the maximal prefix of $o$ in $O\left(s_{1}\right) \cap O\left(s_{2}\right)$ and $o^{\prime} \neq \varepsilon$. Since $o \in O(t) \backslash O\left(s_{2}\right), s_{2}\left(o_{2}\right)=x_{1}$. By assumption (1) this implies that $s_{1}\left(o_{2}\right)=x_{1}$ as well and hence $o^{\prime}=\varepsilon$ which is a contradiction.

Using fact $(*)$ we are able to prove assertion (iii). For a contradiction assume $t_{i} \neq t_{i}^{\prime}$ for $i=1,2, s_{1} t_{1}=s_{2} t_{2}$, and $s_{1} t_{1}^{\prime}=s_{2} t_{2}^{\prime}$, but assertion (iii) does not hold, i.e., there is no $r \in T_{\Sigma}\left(x_{1}\right)$ such that $s_{1} r=s_{2}$ or $s_{1}=s_{2} r$. We distinguish three cases.

Case 1: $s_{1} \leq s_{2}$. Then $O\left(s_{1}\right) \subseteq O\left(s_{2}\right)$ and $\exists o_{1}, o_{2} \in O\left(s_{1}\right), s_{1} / o_{1}=s_{1} / o_{2}=x_{1}$, and $s_{2} / o_{1}=r_{1} \neq s_{2} / o_{2}=r_{2}$. Define $t=s_{1} t_{1}$. Then $t_{1}=t / o_{1}=r_{1} t_{2}$ and $t_{1}=t / o_{2}=$ $r_{2} t_{2}$, and therefore $r_{1} t_{2}=r_{2} t_{2}$. Analogously, we find $r_{1} t_{2}^{\prime}=r_{2} t_{2}^{\prime}$. Hence, by bottom cancellation $r_{1}=r_{2}$ in contradiction to our assumption.

Case 2: $s_{2} \leq s_{1}$. This case is analogous to Case 1 with the role of 1 and 2 exchanged.

Case 3: Neither $s_{1} \leq s_{2}$ nor $s_{2} \leq s_{1}$. By fact $(*), \exists o_{1}, o_{2} \in O\left(s_{1}\right) \cap O\left(s_{2}\right), s_{1} / o_{1}=x_{1}$ and $s_{2} / o_{1}=r_{1} \neq x_{1}$ and $s_{2} / o_{2}=x_{1}$ and $s_{1} / o_{2}=r_{2} \neq x_{1}$.

$s_{1} t_{1}=s_{2} t_{2}$ implies $t_{1}=r_{1} t_{2}$ and $t_{2}=r_{2} t_{1}$. Hence, $t_{1}=r_{1} r_{2} t_{1}$ and so $t_{1}=r_{1} r_{2}$. Similar considerations for $t_{1}^{\prime}$ show that $t_{1}^{\prime}=r_{1} r_{2}$. Hence, $t_{1}=t_{1}^{\prime}$, which is a contradiction. This finishes the proof.

In case the second factors contain variables as well, both bottom cancellation and factorization have a much simpler form. In fact, the same cancellation and factorization rules hold as for the free monoid. We find: 
Corollary 1.2. Assume $s_{1}, s_{2}, t_{1}, t_{2} \in T_{\Sigma}\left(x_{1}\right)$ contain at least one occurrence of $x_{1}$.

(i) Bottom Cancellation. $s_{1} t_{1}=s_{2} t_{1}$ implies $s_{1}=s_{2}$.

(ii) Top Cancellation. $s_{1} t_{1}=s_{1} t_{2}$ implies $t_{1}=t_{2}$.

(iii) Factorization. $s_{1} t_{1}=s_{2} t_{2}$ implies $s_{1} r=s_{2}$ or $s_{1}=s_{2} r$ for some $r \in T_{\Sigma}\left(x_{1}\right)$.

Proof of (i). If $s_{1} t_{1}=s_{2} t_{1}$, then $s_{1} t_{1} t^{\prime}=s_{2} t_{1} t^{\prime}$ for every tree $t^{\prime} \in T_{\Sigma}\left(x_{1}\right)$. Since $t_{1}$ contains an occurrence of $x_{1}, t_{1} t^{\prime} \neq t_{1}$ whenever $t^{\prime} \neq x_{1}$. Hence, we can apply bottom cancellation according to Proposition 1.1(i) and obtain $s_{1}=s_{2}$ according to the assertion.

The proof of assertion (iii) is analogous.

Engelfriet employs the properties stated in Proposition 1.1 to derive a property (T2) which in turn is used to prove the generalization (T1) of Schützenberger's lemma. Since [En] does not contain full proofs of these properties we present them in greater detail. In parallel, we state the corresponding properties for trees that contain at least one occurrence of a variable.

Proposition 1.3 (Engelfriet's Property (T2)).

(i) Assume $s_{i}, t_{i}, u_{i}, v_{i}, w_{i} \in T_{\Sigma}\left(x_{1}\right), i=1,2$, and either $u_{1}$ or $u_{2}$ contains $x_{1}$, $v_{1} \neq w_{1}$ and $v_{2} \neq w_{2}$. Then

$$
\left.\begin{array}{l}
u_{1} v_{1}=u_{2} v_{2} \\
u_{1} w_{1}=u_{2} w_{2} \\
u_{1} t_{1}=u_{2} t_{2} \\
s_{1} v_{1}=s_{2} v_{2} \\
s_{1} w_{1}=s_{2} w_{2}
\end{array}\right\} \text { implies } s_{1} t_{1}=s_{2} t_{2}
$$

(ii) Assume $s_{i}, t_{i}, u_{i}, v_{i} \in T_{\Sigma}\left(x_{1}\right), i=1,2$, and every tree contains at least one occurrence of $x_{1}$. Then

$$
\left.\begin{array}{l}
u_{1} v_{1}=u_{2} v_{2} \\
u_{1} t_{1}=u_{2} t_{2} \\
s_{1} v_{1}=s_{2} v_{2}
\end{array}\right\} \quad \text { implies } s_{1} t_{1}=s_{2} t_{2}
$$

Proof of (i). First assume both $u_{1}$ and $u_{2}$ contain $x_{1}$. By factorization, the first two equations give $u_{1} r=u_{2}$ or $u_{1}=u_{2} r$. Without loss of generality, assume $u_{1} r=u_{2}$. Replacing $u_{2}$ in the first three equations of the assumption with $u_{1} r$ and applying top cancellation we find $v_{1}=r v_{2}, w_{1}=r w_{2}$, and $t_{1}=r t_{2}$. Replacing $v_{1}$ and $w_{1}$ in the last two equations with $r v_{2}$ and $r w_{2}$, respectively, and applying bottom cancellation we obtain $s_{1} r=s_{2}$. Hence we can conclude: $s_{1} t_{1}=s_{1} r t_{2}=s_{2} t_{2}$ which is what we wanted to prove.

Now assume $u_{1}$ does not contain $x_{1}$. Then $u_{1} v_{1}=u_{1} w_{1}=u_{1}$, and hence $u_{2} v_{2}=u_{2} w_{2}=u_{1}$. If $u_{2}$ contained $x_{1}$, then top cancellation would yield $v_{2}=w_{2}$. Since $v_{2} \neq w_{2}$ by assumption, $u_{2}$ cannot contain $x_{1}$ and assertion (i) trivially holds. 
The proof of (ii) is analogous employing the simpler cancellation properties according to Corollary 1.2 .

Proposition 1.4 (Engelfriet's Property (T1)).

(i) Assume $t_{i}, t_{i}^{\prime} \in T_{\Sigma}\left(x_{1}\right), i=0,1,2,3,4$.

$t_{0} \cdots t_{i-1} t_{j} \cdots t_{4}=t_{0}^{\prime} \cdots t_{i-1}^{\prime} t_{j}^{\prime} \cdots t_{4}^{\prime} \quad$ for all $0<i<j \leq 4$

implies $t_{0} t_{1} t_{2} t_{3} t_{4}=t_{0}^{\prime} t_{1}^{\prime} t_{2}^{\prime} t_{3}^{\prime} t_{4}^{\prime}$.

(ii) Assume $t_{i}, t_{i}^{\prime} \in T_{\Sigma}\left(x_{1}\right), i=0,1,2,3$, and every tree contains at least one occurrence of $x_{1}$. Then

$t_{0} \cdots t_{i-1} t_{j} \cdots t_{3}=t_{0}^{\prime} \cdots t_{i-1}^{\prime} t_{j}^{\prime} \cdots t_{3}^{\prime} \quad$ for all $0<i<j \leq 3$

implies $t_{0} t_{1} t_{2} t_{3}=t_{0}^{\prime} t_{1}^{\prime} t_{2}^{\prime} t_{3}^{\prime}$.

Due to the simpler cancellation and factorization properties of trees containing variable occurrences, in (ii) only a subdivision into four factors is needed (as opposed to five in the general case).

In [En] Proposition 1.4(i) is stated with the additional assumption that $t_{0} t_{2} t_{4}=t_{0}^{\prime} t_{2}^{\prime} t_{4}^{\prime}$. However, it is not used in the proof. Removing this assumption enables us to prove a powerful extension of this proposition.

\section{Proof of Proposition 1.4(i).}

Case $1: t_{0}$ or $t_{0}^{\prime}$ contains $x_{1}, t_{4} \neq t_{3} t_{4}$, and $t_{4}^{\prime} \neq t_{3}^{\prime} t_{4}^{\prime}$. We know

$$
\begin{aligned}
& t_{0} t_{4}=t_{0}^{\prime} t_{4}^{\prime}, \\
& t_{0}\left(t_{3} t_{4}\right)=t_{0}^{\prime}\left(t_{3}^{\prime} t_{4}^{\prime}\right), \\
& t_{0}\left(t_{2} t_{3} t_{4}\right)=t_{0}^{\prime}\left(t_{2}^{\prime} t_{3}^{\prime} t_{4}^{\prime}\right), \\
& \left(t_{0} t_{1}\right) t_{4}=\left(t_{0}^{\prime} t_{1}^{\prime}\right) t_{4}^{\prime}, \\
& \left(t_{0} t_{1}\right)\left(t_{3} t_{4}\right)=\left(t_{0}^{\prime} t_{1}^{\prime}\right)\left(t_{3}^{\prime} t_{4}^{\prime}\right) .
\end{aligned}
$$

Proposition 1.3(i) implies $\left(t_{0} t_{1}\right)\left(t_{2} t_{3} t_{4}\right)=\left(t_{0}^{\prime} t_{1}^{\prime}\right)\left(t_{2}^{\prime} t_{3}^{\prime} t_{4}^{\prime}\right)$ which proves the assertion.

Case 2: $t_{0}$ and $t_{0}^{\prime}$ do not contain $x_{1}$. The assertion trivially holds.

Case 3: Either $t_{0}$ or $t_{0}^{\prime}$ contains $x_{1}$ but $t_{4}=t_{3} t_{4}$. It follows that $t_{0}^{\prime} t_{4}^{\prime}=t_{0}^{\prime} t_{3}^{\prime} t_{4}^{\prime}$. Assume $t_{0}^{\prime}$ contains $x_{1}$. Then $t_{4}^{\prime}=t_{3}^{\prime} t_{4}^{\prime}$ by top cancellation. Now the assertion follows from the fact that $t_{0} t_{1} t_{2} t_{4}=t_{0}^{\prime} t_{1}^{\prime} t_{2}^{\prime} t_{4}^{\prime}$ :

$$
t_{0} t_{1} t_{2} t_{3} t_{4}=t_{0} t_{1} t_{2} t_{4}=t_{0}^{\prime} t_{1}^{\prime} t_{2}^{\prime} t_{4}^{\prime}=t_{0}^{\prime} t_{1}^{\prime} t_{2}^{\prime} t_{3}^{\prime} t_{4}^{\prime}
$$


If $t_{0}^{\prime}$ does not contain $x_{1}$, then

$$
\begin{aligned}
t_{0}^{\prime} t_{1}^{\prime} t_{2}^{\prime} t_{3}^{\prime} t_{4}^{\prime} & =t_{0}^{\prime} \\
& =t_{0}^{\prime} t_{1}^{\prime} t_{2}^{\prime} t_{4}^{\prime} \\
& =t_{0} t_{1} t_{2} t_{4} \\
& =t_{0} t_{1} t_{2} t_{3} t_{4}
\end{aligned}
$$

Case 4: Either $t_{0}$ or $t_{0}^{\prime}$ contains $x_{1}$ but $t_{4}^{\prime}=t_{3}^{\prime} t_{4}^{\prime}$. This is analogous to Case 3 .

The proof of statement (ii) is omitted.

Theorem 1.5. Let $K, N \in \mathbb{N}$ and $t_{\kappa, i} \in T_{\Sigma}\left(x_{1}\right)$ for $\kappa=1, \ldots, K$ and $i=0, \ldots, N$.

(i) Assume $N \geq 3 \cdot\left(K^{2}-K\right)$ ! and for every $0<i<j \leq N$ there are $\kappa$, $\kappa^{\prime} \in\{1, \ldots, K\}$ with $\kappa<\kappa^{\prime}$ such that

$t_{\kappa, 0} \cdots t_{\kappa, i-1} t_{\kappa, j} \cdots t_{\kappa, N}=t_{\kappa^{\prime}, 0} \cdots t_{\kappa^{\prime}, i-1} t_{\kappa^{\prime}, j} \cdots t_{\kappa^{\prime}, N}$

Then $t_{\kappa_{0}, 0} \cdots t_{\kappa_{0}, N}=t_{\kappa_{1}, 0} \cdots t_{\kappa_{1}, N}$ for some $\kappa_{0}<\kappa_{1}$ in $\{1, \ldots, K\}$.

(ii) Assume $N \geq 3 \cdot(2 K)$ !, $t_{0,0}, \ldots, t_{0, N}$ is another group of trees in $T_{\Sigma}\left(x_{1}\right)$, and for every $0<i<j \leq N$ some $\kappa \in\{1, \ldots, K\}$ exists such that

$$
t_{0,0} \cdots t_{0, i-1} t_{0, j} \cdots t_{0, N}=t_{\kappa, 0} \cdots t_{\kappa, i-1} t_{\kappa, j} \cdots t_{\kappa, N} .
$$

Then $t_{0,0} \cdots t_{0, N}=t_{\kappa_{0}, 0} \cdots t_{\kappa_{0}, N}$ for some $\kappa_{0} \in\{1, \ldots, K\}$.

Proof. The proof of statement (i) relies on Proposition A from Ramsey Theory which for reasons of self-containedness is presented in the Appendix. For $N \in \mathbb{N}$, let $[N]^{2}$ denote the set $\{\{i, j\} \mid 1 \leq i<j \leq N\}$. Consider the set of "colors" $C:=\left\{\left\{\kappa, \kappa^{\prime}\right\} \mid 1 \leq \kappa<\kappa^{\prime} \leq K\right\}$. By assumption, a $k$-coloring $\rho:[N]^{2} \rightarrow C$ exists where $k=\# C=\frac{1}{2} K(K-1)$ such that, for $i<j, \rho(\{i, j\})=\left\{\kappa, \kappa^{\prime}\right\}$ implies $t_{\kappa, 0} \cdots t_{\kappa, i-1} t_{\kappa, j} \cdots t_{\kappa, N}=t_{\kappa^{\prime}, 0} \cdots t_{\kappa^{\prime}, i-1} t_{\kappa^{\prime}, j} \cdots t_{\kappa^{\prime}, N}$.

According to Proposition A for $m=4$ and $k=\frac{1}{2} K(K-1), 1 \leq \kappa_{0}<\kappa_{1} \leq K$ and $0 \leq i_{1}<\cdots<i_{4} \leq N$ exist such that, for any two $i<j$ from $\left\{i_{1}, i_{2}, i_{3}, i_{4}\right\}$, $t_{\kappa_{0}, 0} \cdots t_{\kappa_{0}, i-1} t_{\kappa_{0}, j} \cdots t_{\kappa_{0}, N}=t_{\kappa_{1}, 0} \cdots t_{\kappa_{1}, i-1} t_{\kappa_{1}, j} \cdots t_{\kappa_{1}, N}$.

Proposition 1.4(i) applied to

$$
\begin{array}{ll}
t_{0}:=t_{\kappa_{0}, 0} \cdots t_{\kappa_{0}, i_{1}-1}, & t_{0}^{\prime}:=t_{\kappa_{1}, 0} \cdots t_{\kappa_{1}, i_{1}-1}, \\
t_{1}:=t_{\kappa_{0}, i_{1}} \cdots t_{\kappa_{0}, i_{2}-1}, & t_{1}^{\prime}:=t_{\kappa_{1}, i_{1}} \cdots t_{\kappa_{1}, i_{2}-1}, \\
t_{2}:=t_{\kappa_{0}, i_{2}} \cdots t_{\kappa_{0}, i_{3}-1}, & t_{2}^{\prime}:=t_{\kappa_{1}, i_{2}} \cdots t_{\kappa_{1}, i_{3}-1}, \\
t_{3}:=t_{\kappa_{0}, i_{3}} \cdots t_{\kappa_{0}, i_{4}-1}, & t_{3}^{\prime}:=t_{\kappa_{1}, i_{3}} \cdots t_{\kappa_{1}, i_{4}-1}, \\
t_{4}:=t_{\kappa_{0}, i_{4}} \cdots t_{\kappa_{0}, N}, & t_{4}^{\prime}:=t_{\kappa_{1}, i_{4}} \cdots t_{\kappa_{1}, N},
\end{array}
$$

yields assertion (i). 
The proof of assertion (ii) is analogous with a set of colors $C:=\{1, \ldots, K\}$.

Theorem 1.5(ii) generalizes Engelfriet's property (T1) in the same sense that Weber's Lemma 2 of [W2] generalizes Schützenberger's lemma. Note that the lower bounds for $N$ in the theorem can be stated more precisely as the Ramsey numbers $R\left(4 ; \frac{1}{2} K(K-1)\right)$ and $R(4 ; K)$, respectively. We stated the results (i) and (ii) slightly weaker but more explicit (with respect to numbers) since we apply them to estimate the complexities of algorithms.

As another application of Ramsey Theory we have:

Proposition 1.6. Assume $s_{1}, s_{2} \in T_{\Sigma}\left(x_{1}\right)$ are different and $m>0$. Then some $N \in \mathbb{N}$ exist such that, for all pairwise different $t_{1}, \ldots, t_{N} \in T_{\Sigma}\left(x_{1}\right)$, an m-element subset $J \subseteq\{1, \ldots, N\}$ and $i \in\{1,2\}$ exist such that the trees $t_{j} s_{i}, j \in J$, are pairwise different.

Proof. For every $j \neq j^{\prime}$ we have, by Proposition 1.1(i), $t_{j} s_{1} \neq t_{j} s_{1}$ or $t_{j} s_{2} \neq t_{j^{\prime}} s_{2}$. Therefore, a 2-coloring $\rho:[N]^{2} \rightarrow\{1,2\}$ exists such that $\rho\left(\left\{j, j^{\prime}\right\}\right)=i$ implies $t_{j} s_{i}$. Now, the assertion follows from Proposition A for $k=2$ (and varying $m$ ).

For trees in $T_{\Sigma}(X)$ the size is more adequately described by a polynomial. Let $\mathbb{Q}^{(1)}[X]:=\mathbb{Q}+\sum_{x \in X} \mathbb{Q} \cdot x$ denote the set of polynomials of degree at most 1 in variables from $X$ with coefficients in $\mathbb{Q}$. We define a map $\omega: T_{\Sigma}(X) \rightarrow \mathbb{Q}^{(1)}[X]$ by

$$
\omega(t):=|t|_{\Sigma}+\sum_{x \in X}|t|_{\{x\}} \cdot x,
$$

i.e., the constant is the $\Sigma$-size of $t$, whereas the coefficient of $x$ is just the number of occurrences of $x$ in $t$. Note that $w(t) \in \mathbb{N}_{0}^{(1)}[X]$.

As for substitutions, maps $\theta: X \rightarrow \mathbb{Q}^{(1)}[X]$ can be extended to maps $\mathbb{Q}^{(1)}[X] \rightarrow \mathbb{Q}^{(1)}[X]$. For $f \in \mathbb{Q}^{(1)}[X], f \theta$ is defined by function composition, i.e., $f \theta$ is the function obtained from $f$ by first applying the functions $x \theta$ and then applying $f$ to the results. As for trees, we call $\theta$ an $X$-substitution or substitution, if $X$ is understood. If $X=\left\{x_{1}, \ldots, x_{m}\right\}$ and $x_{i} \theta=f_{i}$ we denote $f \theta$ by $f\left[f_{1}, \ldots, f_{m}\right]$ and write $f f_{1}$ for $f\left[f_{1}\right]$.

Similar to $T_{\Sigma}\left(x_{1}\right), \mathbb{Q}^{(1)}\left[x_{1}\right]=\mathbb{Q}^{(1)}\left[\left\{x_{1}\right\}\right]$ forms a monoid where the product is defined by

$$
\left(n_{1}+d_{1} x_{1}\right) \cdot\left(n_{2}+d_{2} x_{1}\right):=n_{1}+d_{1}\left(d_{2} x_{1}+n_{2}\right)=\left(n_{1}+d_{1} n_{2}\right)+d_{1} d_{2} x_{1},
$$

and the unity is $x_{1}$.

$\omega$ commutes with substitution. Especially, $\omega: T_{\Sigma}\left(x_{1}\right) \rightarrow \mathbb{Q}^{(1)}\left[x_{1}\right]$ is a monoid morphism, i.e., $\omega(s t)=\omega(s) \omega(t)$ for all $s, t \in T_{\Sigma}\left(x_{1}\right)$. Hence the following proposition holds:

\section{Proposition 1.7.}

(i) If, for $j=1, \ldots, k, t_{j} \in T_{\Sigma}\left(x_{1}\right)$ and $\omega\left(t_{j}\right)=n_{j}+d_{j} x_{1}$, then

$$
\begin{aligned}
\omega\left(t_{1} \cdots t_{k}\right)= & n_{1}+d_{1} n_{2}+\cdots+\left(d_{1} \cdots d_{j-1}\right) n_{j}+\cdots \\
& +\left(d_{1} \cdots d_{k-1}\right) n_{k}+\left(d_{1} \cdots d_{k}\right) \cdot x_{1}
\end{aligned}
$$


(ii) If $t \in T_{\Sigma}\left(x_{1}\right)$ and $\omega(t)=n+d x_{1}$, then

$$
\omega\left(t^{k}\right)=n \cdot \sum_{j=0}^{k-1} d^{j}+d^{k} \cdot x_{1}
$$

With the only exception being Proposition 1.1(iv), statements 1.1-1.6 remain valid for $\mathbb{Q}^{(1)}\left[x_{1}\right]$ as well. We refer to them as $1.1 \mathrm{~S}-1.6 \mathrm{~S}$, respectively. The occurrence of $x_{1}$ in a polynomial $f$ now means that the coefficient of $x_{1}$ is different from 0 . In this case even $f$ is invertible. As an example, we state the version of Proposition 1.1 for polynomials.

Proposition 1.1S. Assume $s_{1}, s_{2}, t_{1}, t_{2}, t_{1}^{\prime}, t_{2}^{\prime} \in \mathbb{Q}^{(1)}\left[x_{1}\right]$ where $s_{i}=n_{i}+d_{i} x_{1}$.

(i) Bottom Cancellation. Assume $t_{1} \neq t_{1}^{\prime}$. Then $s_{1} t_{1}=s_{2} t_{1}$ and $s_{1} t_{1}^{\prime}=s_{2} t_{1}^{\prime}$ implies $s_{1}=s_{2}$.

(ii) Top Cancellation. Assume $x_{1}$ occurs in $s_{1}$, i.e., $d_{1} \neq 0$. Then $s_{1} t_{1}=s_{1} t_{2}$ implies $t_{1}=t_{2}$.

(iii) Factorization. Assume $t_{i} \neq t_{i}^{\prime}$ for $i=1,2$. Then $s_{1} t_{1}=s_{2} t_{2}$ and $s_{1} t_{1}^{\prime}=s_{2} t_{2}^{\prime}$ implies $s_{1} r=s_{2}$ or $s_{1}=s_{2} r$ for some $r \in \mathbb{Q}^{(1)}\left[x_{1}\right]$.

Proof of (iii).

Case 1: $d_{1}=0$ and $d_{2}=0$. Then $s_{1}=s_{2}$.

Case 2: $d_{1} \neq 0$ or $d_{2} \neq 0$. Without loss of generality $d_{1} \neq 0$. Define $s_{1}^{-1}:=$ $d_{1}^{-1} x_{1}+\left(-d_{1}^{-1} n_{1}\right)$ and $r:=s_{1}^{-1} s_{2}$. Then $s_{1} r=s_{2}$.

Note that we in fact proved a somewhat stronger version of (iii), namely that already $s_{1} t_{1}=s_{2} t_{2}$ implies $s_{1} r=s_{2}$ or $s_{1}=s_{2} r$ for some $r \in \mathbb{Q}^{(1)}\left[x_{1}\right]$. However, we only need the version stated in Proposition 1.1S(iii).

In fact, statements 1.2-1.6 are valid for any monoid in which Proposition 1.1(i)-(iii) holds (where " $x_{1}$ occurs in $s$ " should be read as " $\exists t, s t \neq s$ ") with the same proofs as above.

\section{Bottom-Up Finite State Tree Transducers}

In this section we introduce bottom-up FSTs. We define the notion of a computation of a tree transducer quite carefully in order to get precise terminology for the composition and decomposition of subcomputations. A major difficulty when dealing with bottom-up transducers is that during a computation we may substitute a tree $s_{j}$ produced by some subcomputation for a variable $x_{j}$ not occurring in the corresponding output pattern. Thus, $s_{j}$ is dropped and will not be part of the final output. We introduce FSTs which only drop a special symbol 
$\perp$. We show that every FST $M$ can be modified to have this property without changing the translation of $M$ (Proposition 2.1). Moreover, applying Theorems 1.5 and $1.5 \mathrm{~S}$, we prove that it can be decided whether or not an FST is $k$-valued or $k$-size-valued (Theorems 2.2 and 2.3).

For the following, $X$ denotes the fixed denumerable set $\left\{x_{i} \mid i \in \mathbb{N}\right\}$ of variables and $X_{m}=\left\{x_{1}, \ldots, x_{m}\right\}$.

A bottom-up FST is a 5-tuple $M=\left(Q, \Sigma, \Delta, \delta, Q_{\mathrm{F}}\right)$ where:

$Q$ is a finite set of states.

$Q_{\mathrm{F}} \subseteq Q$ is the set of final states.

$\Sigma$ is the signature of input trees.

$\Delta$ is the signature of output trees.

$\delta$, the set of transitions of $M$, is a finite subset of $\bigcup_{m \geq 0} Q \times \Sigma_{m} \times T_{\Delta}\left(X_{m}\right) \times Q^{m}$.

The tree $s$ in a transition $\left(q, a, s, q_{1} \cdots q_{m}\right) \in \delta$ is also called the output pattern of this transition. The transitions in $\delta \cap\{q\} \times \Sigma \times T_{\Delta}(X) \times Q^{*}$ are called $q$ transitions.

$M$ is called finite tree automaton (abbreviated FTA) if, for every transition $\left(q, a, s, q_{1} \cdots q_{m}\right)$ in $\delta$, the output pattern $s$ equals $a\left(x_{1}, \ldots, x_{m}\right)$.

Let $t=a\left(t_{1}, \ldots, t_{m}\right) \in T_{\Sigma}\left(X_{k}\right)$ and $q, q_{1}, \ldots, q_{k} \in Q$. A $\left(q, q_{1} \cdots q_{k}\right)$-computation $\varphi$ of $M$ for $t$ starts at variables $x_{i}$ in the states $q_{i}, i \in\{1, \ldots, k\}$ and consists of $\left(p_{j}, q_{1} \cdots q_{k}\right)$-computations of $M$ for the subtrees $t_{j}, j \in\{1, \ldots, m\}$, together with a transition $\left(q, a, s, p_{1} \cdots p_{m}\right) \in \delta$ for the root. Note that in our notation the resulting state of $\varphi$ at the root of $t$ is written to the left whereas the starting states for the variables are written to the right thus suggesting a bottom-up computation to proceed from the right to the left. This order is chosen according to our (linear) description of trees in Section 1: in $t=a\left(t_{1}, \ldots, t_{m}\right)$, the label $a$ of the root stands to the left of the subtrees $t_{j}$.

Formally, a $\left(q, q_{1} \cdots q_{k}\right)$-computation of $M$ for $t$ is a tree $\varphi$ in $T_{\delta}\left(X_{k}\right)$ with the following properties:

(i) If $t=x_{j}$, then $q=q_{j}$ and $\varphi=x_{j}$.

(ii) If $t=a\left(t_{1}, \ldots, t_{m}\right)$, then $\varphi=\tau\left(\varphi_{1}, \ldots, \varphi_{m}\right)$ for some transition $\tau=$ $\left(q, a, s, p_{1} \cdots p_{m}\right)$ and $\left(p_{j}, q_{1} \cdots q_{k}\right)$-computations $\varphi_{j}$ for $t_{j}$.

A $(q, \varepsilon)$-computation is also called a $q$-computation. A $q$-computation is called accepting iff $q \in Q_{\mathrm{F}}$.

Let $t \in T_{\Sigma}\left(X_{k}\right), o \in O(t)$, and let $\varphi$ be a $\left(q, q_{1} \cdots q_{k}\right)$-computation of $M$ for $t$. The subcomputation of $\varphi$ for the subtree $t / o$ of $t$ with root $o$ is defined as $\varphi / o$. Assume $t=t_{0}\left[t_{1}, \ldots, t_{k}\right]$. Assume $\varphi_{0}$ is a $\left(q, p_{1} \cdots p_{k}\right)$-computation for $t_{0}$, and $\varphi_{j}$ are $\left(p_{i}, q_{1} \cdots q_{m}\right)$-computations for $t_{j}, j=1, \ldots, k$. Then $\varphi:=\varphi_{0}\left[\varphi_{1}, \ldots, \varphi_{k}\right]$ is a $\left(q, q_{1} \cdots q_{m}\right)$-computation of $M$ for $t$. Conversely, if $t_{0}$ contains exactly one occurrence of any $x_{j}$ (i.e., is $X_{k}$-proper), then every $\left(q, q_{1} \cdots q_{m}\right)$-computation $\varphi$ for $t_{0}\left[t_{1}, \ldots, t_{k}\right]$ can be uniquely decomposed into a $\left(q, p_{1} \cdots p_{k}\right)$-computation $\varphi_{0}$ for $t_{0}$, and $\left(p_{j}, q_{1} \cdots q_{m}\right)$-computations $\varphi_{j}$ for $t_{j}, j=1, \ldots, m$ (for suitable states $p_{j}$ ), such that $\varphi=\varphi_{0}\left[\varphi_{1}, \ldots, \varphi_{k}\right]$. 
The output $T(\varphi)$ produced by a $\left(q, q_{1} \cdots q_{k}\right)$-computation $\varphi$ is defined as follows. If $\varphi=x_{j}$, then $T(\varphi):=x_{j}$. If $\varphi=\tau\left(\varphi_{1}, \ldots, \varphi_{k}\right)$ where $\tau=\left(q, a, s, q_{1} \cdots q_{m}\right)$, then $T(\varphi):=s\left[T\left(\varphi_{1}\right), \ldots, T\left(\varphi_{m}\right)\right]$. As an abbreviation, we define $\Omega(\varphi):=\omega(T(\varphi))$.

For convenience (and in abuse of the symbol $\delta$ ) we write $\left(q, t, s, q_{1} \cdots q_{k}\right) \in \delta$ iff there is a $\left(q, q_{1} \cdots q_{k}\right)$-computation $\varphi$ of $M$ for $t$ with $T(\varphi)=s$.

$T_{M}(t):=\{T(\varphi) \mid \varphi$ accepting computation of $M$ for $t\}$ denotes the set of outputs of $M$ for $t ; \Omega_{M}(t):=\{\Omega(\varphi) \mid \varphi$ accepting computation of $M$ for $t\}$ denotes the set of output sizes of $M$ for $t$; $\mathrm{val}_{M}(t):=\# T_{M}(t)$ denotes the number of different outputs of $M$ for $t$; $\operatorname{sval}_{M}(t):=\# \Omega_{M}(t)$ denotes the number of different output sizes of $M$ for $t ; L(M):=\left\{t \in T_{\Sigma} \mid\right.$ there is an accepting computation of $M$ for $\left.t\right\}$ is the language accepted by $M ; T(M):=\left\{(t, s) \mid t \in L(M), s \in T_{M}(t)\right\}$ is the translation defined by $M$ (note: for an FTA $M, T(M)=\{(t, t) \mid t \in L(M)\}) ; \Omega(M):=\left\{(t, s) \mid t \in L(M), s \in \Omega_{M}(t)\right\}$ is the size translation defined by $M$; $\operatorname{val}(M):=\sup \left\{\operatorname{val}_{M}(t) \mid t \in T_{\Sigma}\right\}$ is the valuedness of $M$; and, finally, $\operatorname{sval}(M):=\sup \left\{\operatorname{sval}_{M}(t) \mid t \in T_{\Sigma}\right\}$ is the size-valuedness of $M$.

$M$ is called:

- single-valued if val $(M) \leq 1$,

- $k$-valued if $\operatorname{val}(M) \leq k$,

- finite-valued if $\operatorname{val}(M)<\infty$, and

- infinite-valued if $\operatorname{val}(M)=\infty$.

Analogously, we define the notions single-size-valued, $k$-size-valued, finite-sizevalued, and infinite-size-valued.

For measuring the computational costs of our algorithms relative to the size of an input transducer, we assume that $\Sigma$ and $\Delta$ are fixed in advance and therefore have constant cardinalities and ranks. Throughout this paper the rank of $\Sigma$ is denoted by $L$. Moreover, we assume that every symbol can be stored in one memory cell of a Random Access Machine (with uniform cost measure). Thus, we define the size of $M,|M|$, by

$$
|M|=\sum_{\left(q, a, s, q_{1} \cdots q_{m}\right) \in \delta}(m+|s|+2) .
$$

As a special convention we introduce a new symbol $\perp$ of rank 0 (i.e., $\perp \notin \Delta$ ) and define $\Delta_{\perp}:=\Delta \cup\{\perp\}$. We extend the notion of an FST $M=\left(Q, \Sigma, \Delta, \delta, Q_{\mathrm{F}}\right)$ by extending $\Delta$ to $\Delta_{\perp}$ and allowing $\perp$ as an output pattern of transitions, i.e., for every transition $\left(q, a, s, q_{1} \cdots q_{m}\right)$ of $M$, either $s \in T_{\Delta}\left(X_{m}\right)$ or $s=\perp$. We only consider FSTs $M$ where an output tree $\perp$ is always substituted for a variable which does not occur in the corresponding output pattern. Therefore, $\perp$ does not occur as the leaf of an output tree $s \neq \perp$, i.e., the output of every $\left(q, q_{1} \cdots q_{k}\right)$-computation $\varphi$ of $M$ either equals $\perp$ or is in $T_{\Delta}\left(X_{k}\right)$. The map $\omega$ is extended to trees $t$ in $T_{\Delta_{\perp}}\left(X_{k}\right)$ by $\omega(t):=|t|_{\Delta}+\sum_{j=1}^{k}|t|_{\left\{x_{j}\right\}} \cdot x_{j}$, i.e., we give $\perp$ size 0 . Clearly, this modified map $\omega$ still computes with substitution.

An FST $M=\left(Q, \Sigma, \Delta_{\perp}, \delta, Q_{\mathrm{F}}\right)$ is called reduced if: 
(i) For every $q \in Q$ an accepting computation $\varphi$ of $M$ and a node $o$ in $\varphi$ exist such that $\varphi(o)$ is a $q$-transition.

(ii) There is a subset $U(M) \subseteq Q$ such that, for every transition $\left(q, a, s, q_{1} \cdots q_{m}\right)$ $\in \delta$, the following holds:

if $q \notin U(M)$, then $s \neq \perp$ and $\left(q_{j} \in U(M)\right.$ iff $x_{j}$ does not occur in $\left.s\right)$, and if $q \in U(M)$, then $s=\perp$ and $q_{j} \in U(M)$ for all $j$.

The states in $U(M)$ are exactly those which are used by subcomputations $\varphi$ with $T(\varphi)=\perp$. If a computation has reached some state not in $U(M)$ we can be sure that the output for the corresponding subcomputation is part of the final output.

Proposition 2.1. For every $F S T M=\left(Q, \Sigma, \Delta, \delta, Q_{F}\right)$ there is an FST $M_{r}=$ $\left(Q_{r}, \Sigma, \Delta_{\perp}, \delta_{r}, Q_{r, \mathrm{~F}}\right)$ with the following properties:

(i) $M_{r}$ is reduced.

(ii) $T\left(M_{r}\right)=T(M)$.

$M_{r}$ can be constructed from $M$ in polynomial time.

Proof (Sketch). Define $\bar{M}=\left(\bar{Q}, \Sigma, \Delta_{\perp}, \bar{\delta}, \bar{Q}_{\mathrm{F}}\right)$ where $\bar{Q}:=Q \times\{0,1\}, \quad \bar{Q}_{\mathrm{F}}:=$ $Q_{\mathrm{F}} \times\{1\}$, and $\bar{\delta}$ is defined as follows. Assume $\left(q, a, s, q_{1} \cdots q_{m}\right) \in \delta$.

Then $\left(\langle q, 0\rangle, a, \perp,\left\langle q_{1}, 0\right\rangle \cdots\left\langle q_{m}, 0\right\rangle\right)$ and $\left(\langle q, 1\rangle, a, s,\left\langle q_{1}, \varepsilon_{1}\right\rangle \cdots\left\langle q_{m}, \varepsilon_{m}\right\rangle\right)$ are in $\bar{\delta}$ where $\varepsilon_{j}:=1$ if $x_{j}$ occurs in $s$, and $\varepsilon_{j}:=0$ otherwise.

Then, $T(M)=T(\bar{M})$ and $\bar{M}$ satisfies (ii) of the definition of reducedness with $U(\bar{M})=Q \times\{0\}$. Now eliminate superfluous states and transitions.

Proposition 2.1 can be used to decide in polynomial time whether or not $L(M)$ is empty. The next theorem shows that it can be decided in nondeterministic polynomial time whether $M$ is not $(k-1)$-valued. In the proof reducedness is not needed.

Theorem 2.2. Assume $k>1$ is a fixed constant and $M$ an FST with $n$ states.

(i) $\operatorname{val}(M) \geq k$ iff there is a tree $t$ of depth at most $3 \cdot\left(k^{2}-k\right) ! \cdot n^{k}$ such that $\operatorname{val}_{M}(t) \geq k$.

(ii) It can be decided in nondeterministic polynomial time whether $\operatorname{val}(M) \geq k$.

Proof. (i) Define $N:=3 \cdot\left(k^{2}-k\right)$ !. Assume $t \in T_{\Sigma}$ is a tree of minimal size such that there are accepting computations $\varphi_{1}, \ldots, \varphi_{k}$ of $M$ for $t$ with $T\left(\varphi_{\kappa}\right) \neq T\left(\varphi_{k^{\prime}}\right)$ for $\kappa \neq \kappa^{\prime}$. For a contradiction assume $\operatorname{depth}(t)>N \cdot n^{k}$. Then there are states $p_{1}, \ldots, p_{k} \in Q$, a factorization of $t=t_{0} \cdots t_{N}$ into proper trees $t_{j} \in T_{\Sigma}\left(x_{1}\right)$ and corresponding factorizations $\varphi_{\kappa}=\varphi_{\kappa, 0} \cdots \varphi_{\kappa, N}$ such that $\varphi_{\kappa, j}$ are $\left(p_{\kappa}, p_{\kappa}\right)$-computations for all $\kappa=1, \ldots, k$ and $j=1, \ldots, N-1$. Define $s_{\kappa, j}:=T\left(\varphi_{\kappa, j}\right), \kappa=1, \ldots, k$, $j=0, \ldots, N$. By the minimality of $t$,

$$
\begin{aligned}
& \forall 0<i<j \leq N, \quad \exists \kappa<\kappa^{\prime}, \\
& \quad s_{\kappa, 0} \cdots s_{\kappa, i-1} s_{\kappa, j} \cdots s_{\kappa, N}=s_{\kappa^{\prime}, 0} \cdots s_{\kappa^{\prime}, i-1} s_{\kappa^{\prime}, j} \cdots s_{\kappa^{\prime}, N} .
\end{aligned}
$$


By Theorem 1.5(i), this implies

$$
T\left(\varphi_{\kappa_{0}}\right)=s_{\kappa_{0}, 0} \cdots s_{\kappa_{0}, N}=s_{\kappa_{1}, 0} \cdots s_{\kappa_{1}, N}=T\left(\varphi_{\kappa_{1}}\right) \quad \text { for some } \quad 1 \leq \kappa_{0}<\kappa_{1} \leq k,
$$

a contradiction.

(ii) The first observation is that it suffices to consider only paths of outputs of accepting computations. Paths in a tree $s$ over some alphabet $\Gamma$ start at the root of $s$ and lead to some node $o$. They are denoted by words over the alphabet $B(\Gamma):=\{(a, j) \mid a \in \Gamma, 0 \leq j \leq \rho(a)\}$. The first components of the letters give the labels of the nodes on the path $w$, whereas the second components contain the direction in which $w$ may proceed. Label $(a, 0)$ indicates the endpoint of a path. For $s \in T_{\Gamma}(X)$ we distinguish between sets $b_{j}(s), j>0$, of paths of $s$ leading to nodes labeled with $x_{j}$ and the set $b_{0}(s)$ of paths of $s$ leading to some node not labeled with some $a \in \Gamma$. Formally, we define:

- If $s=x_{j^{\prime}} \in X$, then, for $j \geq 0$,

$$
b_{j}(s):= \begin{cases}\{\varepsilon\} & \text { if } j=j^{\prime} \\ \varnothing & \text { otherwise. }\end{cases}
$$

- If $s=a\left(s_{1}, \ldots, s_{k}\right)$, then

$$
b_{0}(s):=\{(a, 0)\} \cup \bigcup_{\kappa=1}^{k}(a, \kappa) \cdot b_{0}\left(s_{\kappa}\right)
$$

and

$$
b_{j}(s):=\bigcup_{\kappa=1}^{k}(a, \kappa) \cdot b_{j}\left(s_{\kappa}\right) \quad \text { for } \quad j>0 .
$$

Let $w=\left(a_{1}, j_{1}\right) \cdots\left(a_{r}, j_{r}\right)(a, 0) \in B(\Gamma)^{*}$ and $o=j_{1} \cdots j_{r}$. If $w \in b_{0}(s)$, then, in fact, $o \in O(s)$ and $s(o)=a$. Thus, path $w$ of $s$ leads to node $o$. Observe that there is at most one path of $s$ leading to $o \in O(s)$. We have:

(1) For all trees $s_{1}, s_{2} \in T_{\Gamma}, s_{1} \neq s_{2}$ iff paths $w_{i} \in b_{0}\left(s_{i}\right), i=1$, 2, exist leading to some node $o \in O\left(s_{1}\right) \cap O\left(s_{2}\right)$ such that $w_{1} \neq w_{2}$.

Observation (1) follows from the fact that $s_{1} \neq s_{2}$ iff some $o \in O\left(s_{1}\right) \cap O\left(s_{2}\right)$ exists with $s_{1}(o) \neq s_{2}(o)$.

Our second observation is that the paths of the output produced by some computation $\varphi$ are in fact produced by the paths of $\varphi$. For $\pi \in b_{f}(\varphi)$, the set $B(\pi)$ of paths produced by $\pi$ is defined by $B(\pi):=\{\varepsilon\}$ if $\pi=\varepsilon$, and $B(\pi):=b_{\kappa}(u) \cdot B\left(\pi^{\prime}\right)$ if $\pi=(\tau, \kappa) \cdot \pi^{\prime}$ where $\tau=\left(q, a, u, q_{1} \cdots q_{k}\right)$.

We prove:

(2) $b_{j}(T(\varphi))=\bigcup\left\{B(\pi) \mid \pi \in b_{j}(\varphi)\right\}$ for every computation $\varphi$ and $j \geq 0$.

For a proof consider some computation $\varphi$. The sets $b_{j}(T(\varphi))$ inductively can be obtained as follows: 
- If $\varphi=x_{j^{\prime}} \in X$, then, for $j \geq 0$,

$$
b_{j}(T(\varphi))= \begin{cases}\{\varepsilon\} & \text { if } j=j^{\prime}, \\ \varnothing & \text { otherwise. }\end{cases}
$$

- If $\varphi=\tau\left(\varphi_{1}, \ldots, \varphi_{k}\right)$ for $\tau=\left(q, a, u, q_{1} \cdots q_{k}\right) \in \delta$, then

$$
b_{0}(T(\varphi))=b_{0}(u) \cup \bigcup_{\kappa=1}^{k} b_{\kappa}(u) \cdot b_{0}\left(T\left(\varphi_{\kappa}\right)\right)
$$

and

$$
b_{j}(T(\varphi))=\bigcup_{\kappa=1}^{k} b_{k}(u) \cdot b_{j}\left(T\left(\varphi_{k}\right)\right) \quad \text { for } \quad j>0 .
$$

From this, observation (2) follows by induction on the depth of $\varphi$.

For $q \in Q$, let $M_{q}$ denote the FST $M_{q}:=(Q, \Sigma, \Delta, \delta,\{q\})$.

Claim. $\operatorname{val}(M) \geq k$ if and only if $M$ has property $(k$-val):

(k-val) $m \leq L \cdot k(k-1) \cdot N \cdot n^{k}$, some proper $t \in T_{\Sigma}\left(X_{m}\right)$, and $\left(q^{(\kappa)}, p_{1}^{(\kappa)} \cdots p_{m}^{(\kappa)}\right)$ computations $\psi_{\kappa}, \kappa=1, \ldots, k$, of $M$ for $t$ exist such that the following hold:

(a) $|t| \leq L \cdot k(k-1) \cdot N \cdot n^{k}$.

(b) $q^{(\kappa)} \in Q_{\mathrm{F}}$ for all $\kappa$.

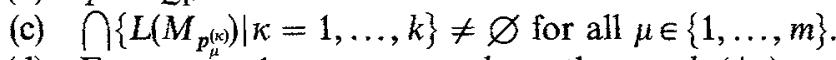

(d) For every $1 \leq \kappa_{1}<\kappa_{2} \leq k$, paths $\pi_{i} \in b_{0}\left(\psi_{\kappa_{i}}\right)$, and $w_{i} \in B\left(\pi_{i}\right)$, $i=1,2$, exist that lead to the same node $o$ but where $w_{1} \neq w_{2}$.

It is easy to construct a nondeterministic polynomial-time algorithm which tests whether $M$ has property ( $k$-val). Therefore, it remains to prove the claim. Direction " $\Leftarrow$ " follows from observations (1) and (2).

For a proof of the remaining implication assume $\operatorname{val}(M) \geq k$. By statement (i) of the theorem, we know that some tree $s \in T_{\Sigma}$ of depth at most $N \cdot n^{k}$ and accepting computations $\varphi_{1}, \ldots, \varphi_{k}$ of $M$ for s exist such that $T\left(\varphi_{\kappa_{1}}\right) \neq T\left(\varphi_{\kappa_{2}}\right)$ for all $\kappa_{1} \neq \kappa_{2}$. Assume $\kappa_{1}<\kappa_{2}$. According to observation (1), paths $w_{\kappa_{1}, \kappa_{2}}^{(i)} \in b_{0}\left(T\left(\varphi_{\kappa_{i}}\right)\right), i=1,2$, exist that lead to the same node but are different. By observation (2), paths $\pi_{\kappa_{1}, \kappa_{2}}^{(i)} \in b_{0}\left(\varphi_{\kappa_{1}}\right)$ exist such that $w_{\kappa_{1}, \kappa_{2}}^{(i)} \in B\left(\pi_{\kappa_{1}, \kappa_{2}}^{(i)}\right)$. Assume $\pi_{\kappa_{1}, \kappa_{2}}^{(i)}$ lead to nodes $o_{\kappa_{1}, \kappa_{2}}^{(i)} \in O\left(\varphi_{\kappa_{i}}\right)=O(s)$. Let $O$ denote the set of all these nodes. We construct $t$ as the smallest tree that contains all paths of $s$ leading to some $o \in O$. Thus, $t$ is obtained by removing all subtrees from $s$ whose roots are not prefixes of any $o \in O$.

Formally, let $R$ denote the set of all nodes $r \in O(s)$ minimal with respect to the prefix ordering that are not prefixes of some $o \in O$. Let $r_{1}, \ldots, r_{m}$ be an enumeration of the nodes in $R$. By construction, $m \leq L \cdot k(k-1) \cdot N \cdot n^{k}$. Now $t$ is obtained from $s$ by replacing the subtrees with roots $r_{j}$ with $x_{j}, j=1, \ldots, m$. According to the definition of $R, t$ is well defined and $X_{m}$-proper. $t$ gives us the decomposition $s=t\left[s_{1}, \ldots, s_{m}\right]$ where $s_{j}:=s / r_{j}$. Let $\varphi_{\kappa}=\psi_{\kappa}\left[\varphi_{\kappa, 1}, \ldots, \varphi_{\kappa, m}\right]$ be the corresponding decomposition of $\varphi_{\kappa}$. Then $m, t$ together with $\psi_{\kappa}, \kappa=1, \ldots, k$, satisfy conditions (a)-(d) of ( $k$-val). This finishes the proof. 
A corresponding result holds for $k$-size-valuedness, but the complexity of the proposed decision procedure is worse. This is due to the fact that size is a "global" property of trees. Therefore, there is no (at least no obvious) way to decide equality of the output sizes produced by two computations for a tree $t$ of polynomial depth-by looking just at a polynomial number of nodes of $t$.

Theorem 2.3. Assume $k>1$ is a fixed constant and $M$ is an FST with $n$ states.

(i) $\operatorname{sval}(M) \geq k$ iff there is a tree $t$ of depth less than $3 \cdot\left(k^{2}-k\right) ! \cdot n^{k}$ such that $\operatorname{sval}_{M}(t) \geq k$.

(ii) It can be decided in polynomial space whether or not $\operatorname{sval}(M) \geq k$.

Proof. The following nondeterministic procedure possible_size computes for a given state $q$ the output size $\Omega(\varphi)$ of a guessed $q$-computation $\varphi$ :

function possible_size(q: state): rational;

begin

guess $m \in\{0, \ldots, L\}$

var $A$ : array $[1 \ldots m]$ of rational;

guess $\left(q, a, s, q_{1} \cdots q_{m}\right) \in \delta$;

for $i:=1$ to $m$ do

$A[i]:=$ possible_size $\left(q_{i}\right)$;

return $\omega(s)[A[1], \ldots, A[m]]$

end;

Assume the guessed computation is a $q$-computation $\varphi$ of $M$ for $t$.

Using the standard stack-based implementation of recursion, the RAM needs a stack size proportional to $L \cdot \operatorname{depth}(t)$ where every stack item contains a pair of a state $p$ and the output size of a subcomputation $\varphi^{\prime}$ of $\varphi$. We have

$$
\Omega\left(\varphi^{\prime}\right) \leq|t| \cdot|M|^{\operatorname{depth}(t)+1} \leq(L \cdot|M|)^{\operatorname{depth}(t)+1} .
$$

Hence, every integer intermediately computed by our RAM has bit length $O(\log (L \cdot|M|) \cdot \operatorname{depth}(t))$. Therefore, the Turing machine space to compute a possible output space for $t$ is polynomial in $|M|$ and $\operatorname{depth}(t)$.

Accordingly, for every constant $k>1$, we can construct a Turing machine which returns the output sizes of $k>1$ accepting computations for a common input tree $t$ which uses space polynomial in $|M|$ and depth $(t)$.

Since it suffices to consider input trees of polynomial depth we can construct a polynomially space-bounded Turing machine that guesses accepting computations $\varphi_{1}, \ldots, \varphi_{k}$ of $M$ for a common (guessed) tree $t$ of polynomial depth, computes the values $\Omega\left(\varphi_{1}\right), \ldots, \Omega\left(\varphi_{k}\right)$, and verifies whether these are pairwise different.

Assume $M$ is a reduced FST. The following notion is crucial in the case distinctions of Sections 3 and 4. State $q \in Q$ is called unique-sized iff $\Omega\left(\varphi_{1}\right)=\Omega\left(\varphi_{2}\right)$ for all $q$-computations $\varphi_{1}, \varphi_{2}$. Define Const $(M):=\{q \in Q \mid q$ is unique-sized $\}$. The states in Const $(M)$ are relevant in what follows since at least two different output sizes are necessary to apply bottom cancellation or factorization according to 
Proposition 1.1S. Observe that, since $M$ is reduced, $q \in U(M)$ implies $\Omega(\varphi)=0$ for every $q$-computation $\varphi$ of $M$. Therefore, $U(M) \subseteq \operatorname{Const}(M)$.

The following proposition summarizes simple properties of the states in Const $(M)$.

Proposition 2.4. Assume $p$ is unique-sized. Then the following holds:

(1) If there is a proper ( $p, q)$-computation, then $q$ is unique-sized as well.

(2) For every proper $(p, p)$-computation $\varphi$ one of the following two possibilities holds:

- $\Omega(\varphi)=0$ and hence $T(\varphi)=\perp$, or

- $\Omega(\varphi)=x_{1}$ and hence $T(\varphi)=x_{1}$.

\section{Necessary Conditions for Finite (Size-) Valuedness}

In this section we introduce three necessary conditions (F0)-(F2) for an FST $M$ to be finite-valued. Property (F0) is implied by Property (F1). We also introduce the corresponding necessary conditions (S0)-(S2) for an FST to be finite sizevalued.

In order to prove that Property (F0) is necessary for $\operatorname{val}(M)<\infty$ and that Properties (S1) and (S2) are necessary for $\operatorname{sval}(M)<\infty$ we introduce (technical) Properties $\left(\mathrm{F}^{\prime}\right),\left(\mathrm{S}^{\prime}\right)$, and $\left(\mathrm{S}^{\prime}\right)$, respectively. The relations among these properties are as follows:

- For $i=1,2, M$ has Property (Si) iff $M$ has Properties (S0) and (Si').

- For $i=0,1,2, M$ has Property (Fi) iff $M$ has Properties (F0') and (Si).

( $\mathrm{F}^{\prime}$ ), (S0), and (S1') correspond to (IV2), (IV3) and (IV1) of the conference version of [W4], respectively. Thus, Property (F0) subsumes Criteria (IV2) and (IV3), and (F1) subsumes all three Criteria. ${ }^{1}$ Our additional Property (S2) (or F2)) has no analogue in the word case.

For Sections 3-5 assume $M=\left(Q, \Sigma, \Delta_{\perp}, \delta, Q_{\mathrm{F}}\right)$ is a reduced FST with $T(M) \subseteq T_{\Sigma} \times T_{\Delta}$ (see Proposition 2.1).

Property (F0). $M$ has Property (F0) for proper $t \in T_{\Sigma}\left(x_{1}\right)$ iff

$$
\left(p, t, s_{1}, p\right),\left(p, t, s_{2}, q\right),\left(q, t, s_{3}, q\right) \in \delta
$$

implies

$$
s_{1} s_{2}=s_{2} s_{3} .
$$

By the assumptions of Property (F0), $u_{k, j}:=s_{1}^{j} s_{2} s_{3}^{k-j-1}$ is the output produced by some $(p, q)$-computation of $M$ for $t^{k}$ for every $1 \leq j \leq k$. Intuitively, the commutation relation (F0) means that the output $s_{1}$ for one copy of $t$ produced in the $p$-loop can be shifted to the output $s_{3}$ produced for $t$ in the $q$-loop. Hence, $u_{k, j}=u_{k, j^{\prime}}$ for every $1 \leq j, j^{\prime} \leq k$.

\footnotetext{
${ }^{1}$ In the journal version of his paper, Weber modified his criteria in the spirit of ours.
} 

with

Property (F0.0) is obtained from Property (F0) by replacing conclusion (F0)

$$
s_{1} \in\left\{\perp, x_{1}\right\} \quad \text { iff } \quad s_{3} \in\left\{\perp, x_{1}\right\} .
$$

It is implied by Property (F0) as can be seen as follows. Without loss of generality, we may assume that $t \neq x_{1}$. First assume $s_{2}$ contains an occurrence of $x_{1}$. Then by reducedness of $M$, also $s_{1}$ and $s_{3}$ contain occurrences of $x_{1}$. Thus, (F0.0) takes the form

$$
s_{1}=x_{1} \quad \text { iff } \quad s_{3}=x_{1} .
$$

If $s_{1}=x_{1}$, then (F0) implies that $s_{2}=s_{2} s_{3}$, and hence $x_{1}=s_{3}$ by top cancellation. If, on the contrary, $s_{3}=x_{1}$, then (F0) implies that $s_{1} s_{2}=s_{2}$, and hence $s_{1}=x_{1}$ by bottom cancellation.

Therefore, assume $s_{2}$ does not contain an occurrence of $x_{1}$. Then $q \in U(M)$ since $M$ is reduced. It follows that $s_{3}=\perp$. Equation (F0) yields that $s_{1} s_{2}=s_{2}$. If $s_{1}$ does not contain an occurrence of $x_{1}$, then by the reducedness of $M, p \in U(M)$. Hence since $t \neq x_{1}, s_{1}=1$. If $s_{1}$ contains $x_{1}, s_{2}$ is a subtree of $s_{1} s_{2}$. Therefore, (F0) can only hold if $s_{1}=x_{1}$.

Property (F1). $\quad M$ has Property (F1) for proper $t \in T_{\Sigma}\left(x_{1}\right)$ iff, for every factorization $t=t_{1} t_{2} t_{3}$, with proper $t_{1}, t_{2}, t_{3} \in T_{\Sigma}\left(x_{1}\right)$,

$$
\begin{aligned}
& \left(p, t_{1}, s_{11}, p_{1}\right),\left(p_{1}, t_{2}, s_{12}, p_{1}\right),\left(p_{1}, t_{3}, s_{13}, p\right), \\
& \left(p, t_{1}, s_{21}, p_{2}\right),\left(p_{2}, t_{2}, s_{22}, p_{2}\right),\left(p_{2}, t_{3}, s_{23}, q\right), \\
& \left(q, t_{1}, s_{31}, p_{3}\right),\left(p_{3}, t_{2}, s_{32}, p_{3}\right),\left(p_{3}, t_{3}, s_{33}, q\right) \in \delta
\end{aligned}
$$

implies

$$
s_{11} s_{12} s_{13} s_{21} s_{23}=s_{21} s_{22} s_{23} s_{31} s_{33} .
$$

Property $(\mathrm{F} 1)$ is less intuitive. It is obtained from $(\mathrm{F} 0)$ by considering a product $t_{1} t_{3}$ and the corresponding factorizations of the $(p, p)-(p, q)$-, and $(q, q)$-computations according to Property (F0). Into these three computations one inserts $p_{i}$-loops for some proper $t_{2}$. Now commutation relation (F1) says that while shifting the output for $t_{1} t_{3}$ from the $p$-loop to the $q$-loop, the output of the $p_{1}$-loop for $t_{2}$ can be shifted to the output of the $p_{2}$-loop for $t_{2}$.

Property (F2). $\quad M$ has Property (F2) for proper $t \in T_{\Sigma}\left(x_{1}\right)$ iff, for every factorization $t=t_{1}\left[x_{1}, t_{2} t_{3}\right]$ with proper $t_{1} \in T_{\Sigma}\left(X_{2}\right)$, proper $t_{2} \in T_{\Sigma}\left(x_{1}\right)$, and $t_{3} \in T_{\Sigma}$,

$$
\begin{aligned}
& \left(p, t_{1}, s_{11}, p p_{1}\right),\left(p_{1}, t_{2}, s_{12}, p_{1}\right),\left(p_{1}, t_{3}, s_{13}, \varepsilon\right), \\
& \left(p, t_{1}, s_{21}, q p_{2}\right),\left(p_{2}, t_{2}, s_{22}, p_{2}\right),\left(p_{2}, t_{3}, s_{23}, \varepsilon\right), \\
& \left(q, t_{1}, s_{31}, q p_{3}\right),\left(p_{3}, t_{2}, s_{32}, p_{3}\right),\left(p_{3}, t_{3}, s_{33}, \varepsilon\right) \in \delta
\end{aligned}
$$


implies

$$
s_{11}\left[s_{21}\left[x_{1}, s_{23}\right], s_{12} s_{13}\right]=s_{21}\left[s_{31}\left[x_{1}, s_{33}\right], s_{22} s_{23}\right] \text {. }
$$

The basic structure of Property (F2) is again the same as for (F0). However, we now consider a tree $t_{1}\left[x_{1}, t_{3}\right]$ for some $\left\{x_{1}, x_{2}\right\}$-proper $t_{1}$. Similar to commutation relation (F1), commutation relation (F2) says that while shifting the output for $t_{1}\left[x_{1}, t_{3}\right]$ from the $p$-loop to the $q$-loop, the output of the $p_{1}$-loop for $t_{2}$ inserted into the $p$-loop can be shifted to the output of the $p_{2}$-loop for $t_{2}$ inserted into the $(p, q)$-computation for $t_{1}\left[x_{1}, t_{3}\right]$.

For $i=0,1,2$, Properties (Si) and (S0.0) are obtained from Property (Fi) and (F0.0) by applying $\omega$ to the output trees in the conclusions (Fi) (resp. (F0.0)). Since $\omega(s)=0$ iff $s=\perp$ and $\omega(s)=x_{1}$ iff $s=x_{1}$ we have:

\section{Fact 0.}

(i) $M$ has Property (F0.0) for $t$ iff $M$ has Property (S0.0) for $t$.

(ii) For $i=0,1,2$, if $M$ has Property (Fi) for $t$, then $M$ also has Property (Si) for $t$.

In what follows whenever we do not specify the tree for which a property holds we mean that it holds for all $x_{1}$-proper trees.

Also, $M$ has Property (F0) for $t$ provided $M$ has Property (F1) for $t$ with $t=t_{1} t_{2} t_{3}$ where $t_{2}=x_{1}$. Hence, if $M$ has Property (F1), then $M$ also has Property (F0). Analogously, if $M$ has Property (S1), then $M$ also has Property (S0).

Now we state the main theorem of this section.

Theorem 3.1. Assume $M$ is a reduced FST. Then the following hold:

(i) If sval $(M)<\infty$, then $M$ has Properties (S1) and (S2).

(ii) If $\operatorname{val}(M)<\infty$, then $M$ has Properties (F1) and (F2).

In order to prove Theorem 3.1, we start by considering Property (S0). Then, we introduce Properties $\left(\mathrm{F}^{\prime}\right),\left(\mathrm{S} 1^{\prime}\right)$, and $\left(\mathrm{S} 2^{\prime}\right)$ which are closely related to (F0), (S1), and (S2), respectively, but are technically more adequate.

Proposition 3.2. If $\operatorname{sval}(M)<\infty$, then $M$ has Property (S0).

Proof. Assume $t \in T_{\Sigma}\left(x_{1}\right)$ is proper, $p, q \in Q$, and $\left(p, t, s_{1}, p\right),\left(p, t, s_{2}, q\right)$, $\left(q, t, s_{3}, q\right) \in \delta$. We show

$$
\omega\left(s_{1} s_{2}\right)=\omega\left(s_{2} s_{3}\right)
$$

Define $\omega\left(s_{i}\right)=n_{i}+d_{i} \cdot x_{1}$ for $i=1,2,3$. Equation (1) is equivalent to (2) and (3):

$$
\begin{aligned}
& n_{1}+\left(d_{1}-1\right) n_{2}=d_{2} n_{3}, \\
& d_{1} d_{2}=d_{2} d_{3} .
\end{aligned}
$$


Since $M$ is reduced, $\left(q, t^{\prime}, s^{\prime}, c\right) \in \delta$ for some trees $t^{\prime}$ and $s^{\prime}$ where $\omega\left(s^{\prime}\right)=n_{4}$. Define $u_{k, j}:=s_{1}^{j} s_{2} s_{3}^{k-j-1}, j=0, \ldots, k-1$. Then $\left(p, t^{k}, u_{k, j}, q\right)$ and $\left(p, t^{k} t^{\prime}, u_{k, j} s^{\prime}, \varepsilon\right) \in \delta$ for all $j$. First assume $p \in U(M)$. Then $q \in U(M)$ since $M$ is reduced. Hence, $s_{1}=s_{2}=s_{3}=\perp$ and $\omega\left(s_{1} s_{2}\right)=0=\omega\left(s_{2} s_{3}\right)$. Now assume $p \notin U(M)$, i.e., $d_{1}>0$. If $q \in \operatorname{Const}(M)$, then $\omega\left(s_{3}^{k-j-1} s^{\prime}\right)=\omega\left(s^{\prime}\right)$. By Proposition 2.4, $\omega\left(s_{3}\right) \in\left\{0, x_{1}\right\}$, i.e., $n_{3}=0$ and $d_{3} \in\{0,1\}$. Hence, the right-hand side of (2) equals 0 . Moreover,

$$
\omega\left(u_{k, j} s^{\prime}\right)=\omega\left(s_{1}^{j} s_{2} s^{\prime}\right)=n_{1} \cdot \sum_{i=0}^{j-1} d_{1}^{i}+d_{1}^{j} \cdot\left[n_{2}+d_{2} n_{4}\right]
$$

by Proposition 1.7. Therefore,

$$
\omega\left(u_{k, j+1} s^{\prime}\right)-\omega\left(u_{k, j} s^{\prime}\right)=d_{1}^{j} \cdot\left[n_{1}+\left(n_{2}+d_{2} n_{4}\right) \cdot\left(d_{1}-1\right)\right] .
$$

If $n_{1}+\left(n_{2}+d_{2} n_{4}\right) \cdot\left(d_{1}-1\right)>0$, then all $\omega\left(u_{k, j} s^{\prime}\right)$ are distinct and $t^{k} t^{\prime}$ has at least $k$ output sizes; since $k$ can be chosen arbitrarily and $M$ is reduced, this implies that $\operatorname{sval}(M)$ is infinite. Consequently, $n_{1}+\left(n_{2}+d_{2} n_{4}\right) \cdot\left(d_{1}-1\right)=0$ from which we deduce that $n_{1}=0$ and $d_{1}=1$ because $s_{2}, s^{\prime} \neq \perp$. Hence, the left-hand side of (2) equals 0 as well. To prove (3), recall that $d_{3} \in\{0,1\}$. If $d_{3}=1$, then $d_{1} d_{2}=$ $d_{2}=d_{2} d_{3}$. If $d_{3}=0$, then $q \in U(M)$. Therefore, $d_{2}=0$ since $M$ is reduced. It follows that $d_{1} d_{2}=0=d_{2} d_{3}$, and (3) holds as well.

Now assume $q \notin \operatorname{Const}(M)$. Hence especially, $q \notin U(M)$ and so $d_{i}>0$ for $i=$ 1, 2, 3. By Proposition 1.7, $\omega\left(u_{k, j}\right)=A_{k, j}+B_{k, j} \cdot x_{1}$ with

$$
A_{k, j}=n_{1} \sum_{i=0}^{j-1} d_{1}^{i}+n_{2} d_{1}^{j}+n_{3} d_{1}^{j} d_{2} \sum_{i=0}^{k-j-2} d_{3}^{i}
$$

and

$$
B_{k, j}=d_{1}^{j} d_{2} d_{3}^{k-j-1} \text {. }
$$

First assume $d_{1} \neq d_{3}$. Then, for every $k$, all the $B_{k, j}$ and hence all the values $\omega\left(u_{k, j}\right)$, $j=0, \ldots, k-1$, are distinct. Since $q \notin \operatorname{Const}(M)$, we have $\left(q, t_{i}, v_{i}, \varepsilon\right) \in \delta$ for $i=$ 1,2 and suitable trees $t_{i}$ such that $\omega\left(v_{1}\right) \neq \omega\left(v_{2}\right)$. Therefore, we can apply Proposition 1.6S and find that, for every $m$, some $k \geq 1$ and $i \in\{1,2\}$ exist such that $t^{k} t_{i}$ has at least $m p$-computations with pairwise different output sizes. Thus, $\operatorname{sval}(M)$ cannot be finite. Therefore, $d_{1}=d_{3}=: d$ and (3) holds. For $A_{k, j}$ we find

$$
A_{k, j}=n_{1} \sum_{i=0}^{j-1} d^{i}+n_{2} d^{j}+n_{3} d_{2} \sum_{i=j}^{k-2} d^{i}
$$

Hence,

$$
A_{k, j+1}-A_{k, j}=d^{j}\left[n_{1}+n_{2}(d-1)-n_{3} d_{2}\right] .
$$

If $n_{1}+n_{2}(d-1)-n_{3} d_{2} \neq 0$, then, for every $k$, all the values $A_{k, j}$ and hence all $\omega\left(u_{k, j}\right), j=0, \ldots, k-1$, are distinct which as above allows us to construct a contradiction to $\operatorname{sval}(M)<\infty$. Therefore, $n_{1}+n_{2}(d-1)-n_{3} d_{2}=0$ which gives (2).

The next property is crucial to "recover" Properties (Fi) from Properties (Si). 
Property (F0'). $M$ has Property (F0') for proper $t \in T_{\Sigma}\left(x_{1}\right)$ iff

$\left(p, t, s_{1}, p\right),\left(p, t, s_{2}, q\right),\left(p, t, s_{3}, q\right),\left(q, t, s_{4}, q\right) \in \delta$,

$s_{1} \notin\left\{\perp, x_{1}\right\}$ and $\omega\left(s_{2}\right)=\omega\left(s_{3}\right) \quad$ implies $s_{2}=s_{3}$.

$M$ has Property $\left(\mathrm{F}^{\prime}\right)$ iff $M$ has Property (F0') for all $x_{1}$-proper trees $t$.

Proposition 3.3. If $\operatorname{val}(M)<\infty$, then $M$ has Property $\left(\mathrm{F0}^{\prime}\right)$.

Proof. Assume val $(M)<\infty$, and let $t \in T_{\Sigma}\left(x_{1}\right)$ be proper, $\left(p, t, s_{1}, p\right),\left(p, t, s_{2}, q\right)$, $\left(p, t, s_{3}, q\right),\left(q, t, s_{4}, q\right) \in \delta, s_{1} \notin\left\{\perp, x_{1}\right\}$, and $\omega\left(s_{2}\right)=\omega\left(s_{3}\right)$. We show $s_{2}=s_{3}$.

Since $\operatorname{val}(M)<\infty, M$ has Property (S0) by Proposition 3.2 and hence also (F0.0) by Fact 0 . Therefore, $s_{1} \notin\left\{\perp, x_{1}\right\}$ implies $s_{4} \notin\left\{\perp, x_{1}\right\}$. Hence, since $M$ is reduced, $s_{i}$ contains at least one occurrence of $x_{1}$ for all $i=1,2,3,4$. Also, since $M$ is reduced, trees $t^{\prime} \in T_{\Sigma}$ and $s^{\prime} \in T_{\Delta}$ exist with $\left(q, t^{\prime}, s^{\prime}, \varepsilon\right) \in \delta$. Define $s:=s_{4} s^{\prime}$. Since $s_{4} \notin\left\{\perp, x_{1}\right\}, s \neq s^{\prime}$. Note that $\left(q, t t^{\prime}, s, \varepsilon\right) \in \delta$. For $k \geq 1$ consider $u_{k, j}:=s_{1}^{j} s_{2} s_{4}^{k-j-1}$, $j=0, \ldots, k-1$. Then $\left(p, t^{k}, u_{k, j}, q\right) \in \delta$ for all $j$. Assume, for every $k$, that all the outputs $u_{k, j}, j=0, \ldots, k-1$, are distinct. Then, by Proposition 1.6, for every $m$ some $k$ exists such that at least $m p$-computations exist either for $t^{k} t^{\prime}$ or for $t^{k+1} t^{\prime}$ which produce pairwise different output values. Since $\operatorname{val}(M)<\infty$, this is a contradiction. Therefore, $k>1$ and $0 \leq j_{1}<j_{2}<k$ exist such that $u_{k, j_{1}}=u_{k, j_{2}}$. By top and bottom cancellation we find

$$
s_{1}^{j} s_{2}=s_{2} s_{4}^{j},
$$

where $j=j_{2}-j_{1}$. By induction on $n$ this implies $s_{1}^{j n} s_{2}=s_{2} s_{4}^{j n}$ for all $n \in \mathbb{N}$. By Corollary 1.2(iii), either $s_{2} r=s_{1}^{j n}$ or $s_{2}=s_{1}^{j n} r$ for some suitable tree $r$. Since $s_{1}$ is proper but different from $x_{1}$, we have $\left|s_{1}^{j n}\right|_{\Sigma} \geq j n$. Hence, for sufficiently large $n, s_{1}^{j n}$ cannot be a prefix of $s_{2}$. Therefore,

$$
\exists n_{1} \forall n \geq n_{1} \exists r, \quad s_{2} r=s_{1}^{j n} .
$$

Analogously, we find some $j^{\prime}$ such that

$$
\exists n_{2} \forall n \geq n_{2} \exists r^{\prime}, \quad s_{3} r^{\prime}=s_{1}^{j^{\prime n}} .
$$

Choosing in (2) and (3) a sufficiently large common multiple of $j$ and $j^{\prime}$ as an exponent of $s_{1}$ we conclude that $s_{2} r=s_{3} r^{\prime}$ for proper trees $r, r^{\prime} \in T_{\Delta}\left(x_{1}\right)$. Hence by Corollary 1.2(ii) and Proposition 1.1(iv), $s_{2}=s_{3}$.

\section{Proposition 3.4.}

(i) Assume te $T_{\Sigma}\left(x_{1}\right)$ is proper. If $M$ has Property (F0) for $t$, then $M$ has Property $\left(\mathrm{F0}^{\prime}\right)$ for $t$.

(ii) Assume $M$ has Property (F0'). Then, for $i=0,1,2, M$ has Property (Si) iff $M$ has Property (Fi).

Proof. The proof of assertion (i) is analogous to the proof of Proposition 3.3: just observe that $s_{1} s_{2}=s_{2} s_{3}$ implies that $s_{1}^{j} s_{2}=s_{2} s_{3}^{j}$ for all $j$. 
(ii) If $M$ has Property (Fi), then certainly also Property (Si). Conversely, assume $M$ has Property ( $\mathrm{S} 0)$ and $\left(\mathrm{F}^{\prime}\right)$. Assume $t \in T_{\Sigma}\left(x_{1}\right)$ is proper and $\left(p, t, s_{1}, p\right)$, $\left(p, t, s_{2}, q\right),\left(q, t, s_{3}, q\right) \in \delta$.

We show $s_{1} s_{2}=s_{2} s_{3}$. By Fact $0, M$ has Property (F0.0). Therefore, assume $s_{1} \notin\left\{\perp, x_{1}\right\}$. Certainly, $\left(p, t t, s_{1} s_{1}, p\right),\left(p, t t, s_{1} s_{2}, q\right),\left(p, t t, s_{2} s_{3}, q\right),\left(q, t t, s_{3} s_{3}, q\right) \in \delta$ where $s_{1} s_{1} \notin\left\{\perp, x_{1}\right\}$. Since $M$ has Property (S0), $\omega\left(s_{1} s_{2}\right)=\omega\left(s_{2} s_{3}\right)$. Since $M$ has Property $\left(\mathrm{F}^{\prime}\right)$ for $t t, s_{1} s_{2}=s_{2} s_{3}$-which we wanted to prove.

For proofs of the assertion with $i=1$ or 2 , we only treat the case where $s_{12} \in\left\{\perp, x_{1}\right\}$. The remaining parts of the proofs are analogous to the proof for $i=0$. So assume $M$ has Property (S1). Especially, $M$ also has Property (S0.0) and therefore also Property (F0.0). Let $t=t_{1} t_{2} t_{3} \neq x_{1}$ for $x_{1}$-proper trees $t_{i}$, $i=1,2,3$, let

$$
\begin{aligned}
& \left(p, t_{1}, s_{11}, p_{1}\right),\left(p_{1}, t_{2}, s_{12}, p_{1}\right),\left(p_{1}, t_{3}, s_{13}, p\right), \\
& \left(p, t_{1}, s_{21}, p_{2}\right),\left(p_{2}, t_{2}, s_{22}, p_{2}\right),\left(p_{2}, t_{3}, s_{23}, q\right), \\
& \left(q, t_{1}, s_{31}, p_{3}\right),\left(p_{3}, t_{2}, s_{32}, p_{3}\right),\left(p_{3}, t_{3}, s_{33}, q\right) \in \delta,
\end{aligned}
$$

and assume $s_{11} s_{12} s_{13} \in\left\{\perp, x_{1}\right\}$. First assume $s_{11} s_{12} s_{13}=\perp$. Then both $p$ and $q$ are from $U(M)$. Therefore, $s_{21} s_{22} s_{23} s_{31} s_{33}=\perp$ as well, and the conclusion according to Property (F1) holds. Now assume $s_{11} s_{12} s_{13}=x_{1}$. Then, especially, $s_{12}=x_{1}$. If also $s_{22} \in\left\{\perp, x_{1}\right\}$, then $s_{21} s_{22} s_{23} s_{31} s_{33}=s_{21} s_{23} s_{31} s_{33}$, and the conclusion of (F1) follows since $M$ has Property (F0.0) for $t_{1} t_{3}$. For a contradiction assume $s_{22} \notin\left\{\perp, x_{1}\right\}$, i.e., $\omega\left(s_{22}\right) \notin\left\{0, x_{1}\right\}$. Denote $\omega\left(s_{i j}\right)$ by $g_{i j}$. Let $g_{21}=n_{1}+d_{1} x_{1}$, $g_{22}=n_{2}+d_{2} x_{1}$, and $g_{23} g_{31} g_{33}=n_{3}+d_{3} x_{1}$. Since $M$ has Property (S1) for $t_{1} t_{2}^{k} t_{3}$, we have, for every $k>0$,

$$
g_{11} g_{13} g_{21} g_{23}=g_{11} g_{12}^{k} g_{13} g_{21} g_{23}=g_{21} g_{22}^{k} g_{23} g_{31} g_{33}=A_{k}+B_{k} \cdot x_{1},
$$

where

$$
A_{k}=n_{1}+d_{1} n_{2} \cdot \sum_{i=0}^{k-1} d_{2}^{i}+d_{1} d_{2}^{k} n_{3} .
$$

It follows that

$$
0=A_{k}-A_{k-1}=d_{1} n_{2} d_{2}^{k-1}+d_{1} d_{2}^{k-1}\left(d_{2}-1\right) \cdot n_{3} .
$$

$g_{21}$ contains an occurrence of $x_{1}$. Therefore, $d_{1} \neq 0$. Hence, $n_{2}=0$ and $d_{2} \in\{0,1\}$ in contradiction to our assumption.

Now assume $M$ has Property (S2). Especially, $M$ has Property (S0.0) and therefore also Property (F0.0)-at least for all trees containing a symbol $a \in \Sigma$ of rank $>1$. Let $t=t_{1}\left[x_{1}, t_{2} t_{3}\right]$ for $X_{2}$-proper tree $t_{1}$ and $x_{1}$-proper tree $t_{2}$. Let

$$
\begin{aligned}
& \left(p, t_{1}, s_{11}, p p_{1}\right),\left(p_{1}, t_{2}, s_{12}, p_{1}\right),\left(p_{1}, t_{3}, s_{13}, \varepsilon\right), \\
& \left(p, t_{1}, s_{21}, q p_{2}\right),\left(p_{2}, t_{2}, s_{22}, p_{2}\right),\left(p_{2}, t_{3}, s_{23}, \varepsilon\right), \\
& \left(q, t_{1}, s_{31}, q p_{3}\right),\left(p_{3}, t_{2}, s_{32}, p_{3}\right),\left(p_{3}, t_{3}, s_{33}, \varepsilon\right) \in \delta,
\end{aligned}
$$


and assume $s_{11}\left[x_{1}, s_{12} s_{13}\right] \in\left\{\perp, x_{1}\right\}$. First assume $s_{11}\left[x_{1}, s_{12} s_{13}\right]=\perp$. Then both $p$ and $q$ are from $U(M)$. Therefore, $s_{21}\left[s_{31}\left[x_{1}, s_{33}\right], s_{22} s_{23}\right]=\perp$ as well, and the conclusion according to Property (F1) holds. Now assume $s_{11}\left[x_{1}, s_{12} s_{13}\right]=x_{1}$. Then, especially, $s_{12}=\perp$. Again, if also $s_{22} \in\left\{\perp, x_{1}\right\}$, then

$$
s_{21}\left[s_{31}\left[x_{1}, s_{33}\right], s_{22} s_{23}\right]=s_{21}\left[s_{31}\left[x_{1}, s_{33}\right], s_{23}\right] \text {, }
$$

and the conclusion of (F1) follows since $M$ has Property (F0.0) for $t_{1}\left[x_{1}, t_{3}\right]$. For a contradiction assume $s_{22} \notin\left\{1, x_{1}\right\}$, i.e., $\omega\left(s_{22}\right) \notin\left\{0, x_{1}\right\}$. Denote $\omega\left(s_{i j}\right)$ by $g_{i j}$. Let $g_{21}=n_{1}+d_{1} x_{1}+e x_{2}, g_{22}=n_{2}+d_{2} x_{1}, g_{23}=n_{3}$, and $g_{31}\left[x_{1}, g_{33}\right]=n_{4}+d_{4} x_{1}$. Since $M$ has Property (S1) for $t_{1}\left[x_{1}, t_{2}^{k} t_{3}\right]$, we have, for every $k>0$,

$$
\begin{aligned}
g_{11}\left[g_{21}\left[x_{1}, g_{23}\right], g_{13}\right] & =g_{11}\left[g_{21}\left[x_{1}, g_{23}\right], g_{12}^{k} g_{13}\right]=g_{21}\left[g_{31}\left[x_{1}, g_{33}\right], g_{22}^{k} g_{23}\right] \\
& =g_{21} g_{22}^{k} g_{23} g_{31} g_{33}=A_{k}+B_{k} \cdot x_{1},
\end{aligned}
$$

where

$$
A_{k}=n_{1}+d_{1} n_{4}+e n_{2} \cdot \sum_{i=0}^{k-1} d_{2}^{j}+e d_{2}^{k} n_{3} .
$$

It follows that

$$
0=A_{k}-A_{k-1}=e n_{2} d_{2}^{k-1}+e d_{2}^{k-1}\left(d_{2}-1\right) \cdot n_{3} .
$$

$g_{21}$ contains an occurrence of $x_{2}$. Therefore, $e \neq 0$. Hence, $n_{2}=0$ and $d_{2} \in\{0,1\}$ in contradiction to our assumption.

Property (S1). $M$ has Property $\left(\mathrm{S} 1^{\prime}\right)$ for proper tree $t \in T_{\Sigma}\left(x_{1}\right)$ iff, for every factorization $t=t_{1} t_{2} t_{3}, t_{i}$ proper,

$$
\begin{aligned}
& \left(p, t_{1}, s_{11}, p_{1}\right),\left(p_{1}, t_{2}, s_{12}, p_{1}\right),\left(p_{1}, t_{3}, s_{13}, p\right), \\
& \left(p, t_{1}, s_{21}, p_{2}\right),\left(p_{2}, t_{2}, s_{22}, p_{2}\right),\left(p_{2}, t_{3}, s_{23}, q\right), \\
& \left(q, t_{1}, s_{31}, p_{3}\right),\left(p_{3}, t_{2}, s_{32}, p_{3}\right),\left(p_{3}, t_{3}, s_{33}, q\right) \in \delta,
\end{aligned}
$$

with $\omega\left(s_{i j}\right)=g_{i j}$ implies:

(i) If $q \in \operatorname{Const}(M)$, then $g_{12}, g_{22} \in\left\{0, x_{1}\right\}$.

(ii) If $q \notin \operatorname{Const}(M)$, then

$$
g_{11} g_{12} g_{11}^{-1}=g_{21} g_{22} g_{21}^{-1} \text {. }
$$

Recall that if $q$ is not unique-sized, then the output sizes produced by proper $(q, q)$ - or $(p, q)$-computations depend on $x_{1}$. Hence, by reducedness of $M$, $g_{11}$ and $g_{21}$ depend on $x_{1}$ as well. Therefore, the inverses occurring in (S1') indeed exist. Observe that $g_{i 3}$ or $g_{3 j}$ do not occur in (S1'). 
Fact 1. Assume $A, B \in \mathbb{Q}^{(1)}\left[x_{1}\right]$ where $A=a_{0}+a_{1} \cdot x_{1}$ and $B=b_{0}+b_{1} \cdot x_{1}$. Then:

(1) $B^{-1} A=\left(1 / b_{1}\right) \cdot\left[-b_{0}+a_{0}\right]+\left(a_{1} / b_{1}\right) \cdot x_{1}$.

(2) $B A B^{-1}=b_{1} \cdot a_{0}+b_{0} \cdot\left(1-a_{1}\right)+a_{1} \cdot x_{1}$.

Fact 2. Assume $q \notin \operatorname{Const}(M)$ :

(i) Then $\left(\mathrm{S1}^{\prime}\right)$ is equivalent to

$$
\left[g_{21} g_{22} g_{23}\right]^{-1} \cdot\left[g_{11} g_{12} g_{13}\right]=\left[g_{21} g_{23}\right]^{-1} \cdot\left[g_{11} g_{13}\right] \text {. }
$$

(ii) If $g_{i 1}=a_{i}+e_{i} \cdot x_{1}$ and $g_{i 2}=n_{i}+d_{i} \cdot x_{1}$, then (S1') is equivalent to the two equations:

$\left(d_{1}-1\right)\left[a_{2}-a_{1}\right]=e_{2} n_{2}-e_{1} n_{1}$

and

$d_{1}=d_{2}$.

Proof. By top and bottom cancellation, $\left(\mathrm{S} 1^{\prime \prime}\right)$ is equivalent to

$$
\left(g_{21} g_{22}\right)^{-1} \cdot g_{11} g_{12}=g_{21}^{-1} g_{11}
$$

which is equivalent to (S1'). This proves (i).

The proof of (ii) follows by simple calculations from Fact 1.

\section{Proposition 3.5.}

(i) Assume $t \in T_{\Sigma}\left(x_{1}\right)$ is proper:

If $M$ has Property (S1') for $t$, then $M$ has Property (S0) for $t$.

If $M$ has Property (S0) for $t$ and $t_{1} t_{3}$ whenever $t=t_{1} t_{2} t_{3}$ for proper $t_{1}, t_{2}$, $t_{3}$, then $M$ has Property (S1) for $t$ iff $M$ has Property $\left(\mathrm{S} 1^{\prime}\right)$ for $t$.

(ii) If $\operatorname{sval}(M)<\infty$, then $M$ has Property (S1').

Proof. (i) Assume $\left(p, t, s_{1}, p\right),\left(p, t, s_{2}, q\right),\left(q, t, s_{3}, q\right) \in \delta$ and let $g_{i}:=\omega\left(s_{i}\right)$ for $i=$ $1,2,3$. Consider the decomposition $t t=t_{1} t_{2} t_{3}$ where $t_{1}:=x_{1}$ and $t_{2}:=t_{3}:=t$. If $q \in \mathrm{Const}(M)$, then the conclusion of (S1') gives $g_{1}, g_{3} \in\left\{0, x_{1}\right\}$ in accordance with (S0). If $q \notin \operatorname{Const}(M)$, then the conclusion of (S1') gives $x_{1} g_{1} x_{1}^{-1}=g_{2} g_{3} g_{2}^{-1}$. Since $x_{1}^{-1}=x_{1}$ this implies the conclusion of (S0) also in this case.

For the second part of (i) we show that the conclusion according to (S1) is equivalent to the conclusion according to $\left(\mathrm{S} 1^{\prime}\right)$. Assume $p, p_{i}, q, s_{i j}$ are chosen according to the assumptions of Properties (S1') and (S1) and $g_{i j}:=\omega\left(s_{i j}\right)$.

By assumption, $M$ has Property (S0) for $t_{1} t_{3}$ and $t_{1} t_{2} t_{3}$. Therefore,

$$
\begin{aligned}
& g_{11} g_{13} g_{12} g_{23}=g_{21} g_{23} g_{31} g_{33}, \\
& g_{11} g_{12} g_{13} g_{21} g_{22} g_{23}=g_{21} g_{22} g_{23} g_{31} g_{32} g_{33} \text {. }
\end{aligned}
$$


First assume $q \in$ Const $(M)$. Then $g_{31} g_{32} g_{33} \in\left\{0, x_{1}\right\}$ by Proposition 2.4. From (2) we conclude (by the reducedness of $M$ ) that also $g_{11} g_{12} g_{13} \in\left\{0, x_{1}\right\}$. Hence (again by the reducedness of $M$ ), especially, $g_{12} \in\left\{0, x_{1}\right\}$. If $g_{11} g_{12} g_{13}=0$, then $p \in U(M)$, and the assertion follows from the reducedness of $M$. If $g_{11} g_{12} g_{13}=x_{1}$, then $g_{11}=g_{12}=g_{13}=x_{1}$. The conclusion of (S1) has the form

$$
g_{21} g_{23}=g_{21} g_{22} g_{23} \text {. }
$$

This is (by the reducedness of $M$ ) equivalent to

$$
g_{22} \in\left\{0, x_{1}\right\}
$$

according to $\left(\mathrm{S}^{\prime}\right)$.

Now, assume $q \notin \operatorname{Const}(M)$. Then all $g_{i j}$ are invertible, and we have

$$
\left(g_{11} g_{12} g_{13}\right) g_{21} g_{23}=\left(g_{21} g_{22} g_{23}\right) g_{31} g_{33}
$$

iff

$$
\left(g_{11} g_{12} g_{11}^{-1}\right) g_{11} g_{13} g_{21} g_{23}=\left(g_{21} g_{22} g_{21}^{-1}\right) g_{21} g_{23} g_{31} g_{33}
$$

iff

$$
g_{11} g_{12} g_{11}^{-1}=g_{21} g_{22} g_{21}^{-1}
$$

provided (1) holds.

The last equation is the conclusion according to ( $\left.\mathrm{S} 1^{\prime}\right)$.

(ii) Again assume $p, p_{i}, q, s_{i j}$ are chosen according to the assumptions of Property $\left(\mathbf{S 1}^{\prime}\right)$ and $g_{i j}:=\omega\left(s_{i j}\right)$.

For $k, j \geq 0$, define $t^{(j)}:=t_{1} t_{2}^{j} t_{3}$ and $\bar{t}^{(k)}:=t^{(k)} t^{(k-1)} \cdots t^{(0)}$. Define $v_{i}^{(j)}:=s_{i 1} s_{i 2}^{j} s_{i 3}$, $i=1,2,3, \quad$ and $\quad u_{k, j}:=v_{1}^{(k)} \cdots v_{1}^{(j+1)} v_{2}^{(j)} v_{3}^{(j-1)} \cdots v_{3}^{(0)}, 0 \leq j \leq k-1$. We have $\left(p, \bar{t}^{(k)}, u_{k, j}, q\right) \in \delta$ for all $j$. For convenience, we write $h_{i}^{(j)}$ for $\omega\left(v_{i}^{(j)}\right)$, i.e., $h_{i}^{(j)}=g_{i 1} g_{i 2}^{j} g_{i 3}$.

First assume $q \in \operatorname{Const}(M)$. Then $\omega\left(s_{31} s_{32} s_{33}\right) \in\left\{0, x_{1}\right\}$ by Proposition 2.4, and $\omega\left(s_{11} s_{12} s_{13}\right) \in\left\{0, x_{1}\right\}$ by Proposition 3.2. If $p \in U(M)$, then $s_{i j}=\perp$ for all $i, j$ since $M$ is reduced. Therefore, $g_{i j}=\omega\left(s_{i j}\right)=0$ for all $i, j$, and the assertion trivially holds. If $p \notin U(M)$, then $g_{11} g_{12} g_{13}=x_{1}$. Especially, $g_{12}=x_{1}$. Since $M$ is reduced, $t^{\prime} \in T_{\Sigma}$ and $s \in T_{\Delta} \cup\{\perp\}$ exist with $\left(q, t^{\prime}, s, \varepsilon\right) \in \delta$. Assume $g_{2 v}=a_{v}+b_{v} \cdot x_{1}$ and $\omega(s)=m$. Then we have

$$
\omega\left(u_{k, j} s\right)=\omega\left(v_{2}^{(j)} s\right)=a_{1}+b_{1} a_{2} \sum_{i=0}^{j-1} b_{2}^{i}+b_{1} b_{2}^{j} \cdot\left[a_{3}+b_{3} m\right] .
$$

Therefore,

$$
\omega\left(u_{k, j+1} s\right)-\omega\left(u_{k, j} s\right)=b_{1} b_{2}^{j} \cdot\left[a_{2}+\left(a_{3}+b_{3} m\right) \cdot\left(b_{2}-1\right)\right] \geq 0 .
$$

If this is greater than 0 , then all $\omega\left(u_{k, j} s\right)$ are distinct which gives a contradiction to $\operatorname{sval}(M)<\infty$. Hence, $b_{1} b_{2}^{j} \cdot\left[a_{2}+\left(a_{3}+b_{3} m\right) \cdot\left(b_{2}-1\right)\right]=0$. Then two cases can occur. Either $b_{1} b_{2}=0$ which implies $p_{2} \in U(M)$, then $g_{22}=0$ in accordance with Property $\left(\mathbf{S} 1^{\prime}\right)$, or $b_{1} b_{2}>0$, then $\left[a_{2}+\left(a_{3}+b_{3} m\right) \cdot\left(b_{2}-1\right)\right]=0$. We deduce that $a_{2}=0$. Hence $g_{22} \in\left\{0, x_{1}\right\}$ and the conclusion of (S1') holds. 
Now assume $q \notin \operatorname{Const}(M)$. Then all $g_{i j}$ are invertible. Applying Proposition 3.2 to $t^{(j)}$, we obtain

$$
h_{1}^{(j)} h_{2}^{(j)}=h_{2}^{(j)} h_{3}^{(j)}
$$

for all $j$. Moreover, we have

$$
\begin{aligned}
\omega\left(u_{k, j+1}\right) & =h_{1}^{(k)} \cdots h_{1}^{(j+2)} h_{2}^{(j+1)} h_{3}^{(j)} \cdots h_{3}^{(0)} \\
& =h_{1}^{(k)} \cdots h_{1}^{(j+2)}\left(h_{2}^{(j+1)} h_{3}^{(j)}\right) h_{3}^{(j-1)} \cdots h_{3}^{(0)} .
\end{aligned}
$$

Since $h_{i}^{(j+1)}=\left(g_{i 1} g_{i 2} g_{i 1}^{-1}\right) h_{i}^{(j)}$, we conclude

$$
\begin{aligned}
h_{2}^{(j+1)} h_{3}^{(j)} & =\left(g_{21} g_{22} g_{21}^{-1}\right) h_{2}^{(j)} h_{3}^{(j)} \\
& =\left(g_{21} g_{22} g_{21}^{-1}\right) h_{1}^{(j)} h_{2}^{(j)} \quad \text { by }(1) \\
& =A h_{1}^{(j+1)} h_{2}^{(j)},
\end{aligned}
$$

where

$$
A=\left(g_{21} g_{22} g_{21}^{-1}\right)\left(g_{11} g_{12} g_{11}^{-1}\right)^{-1} .
$$

Substituting this into (2) we find

$$
\omega\left(u_{k, j+1}\right)=\left(B_{k, j} A B_{k, j}^{-1}\right) \omega\left(u_{k, j}\right)
$$

for $B_{k, j}=h_{1}^{(k)} \cdots h_{1}^{(j+2)}$ which is a polynomial from $\mathbb{N}_{0}^{(1)}\left[x_{1}\right]$.

If $A=x_{1}$, then the conclusion of (S1') holds. Hence for a contradiction, assume $A \neq x_{1}$. Let $A=a_{0}+a_{1} \cdot x_{1}$ and $\omega\left(u_{k, j}\right)=U_{k, j}+V_{k, j} \cdot x_{1}$.

Case 1: $a_{1} \neq 1$. Then $V_{k, j}=a_{1}^{j-1} \cdot V_{k, 0}$ by Fact 1 and (3). It follows that all the output sizes $\omega\left(u_{k, j}\right)$ are different. Since $q \notin \operatorname{Const}(M)$ we deduce from Proposition $1.6 \mathrm{~S}$ that $\operatorname{sval}(M)=\infty$ in contradiction to our assumption.

Case 2: $a_{1}=1$. Since $A \neq x_{1}$ this implies that $a_{0} \neq 0$. Let $B_{k, j} A B_{k, j}^{-1}=b_{k, j}+x_{1}$. Then by Fact 1 and (3), $U_{k, j+1}=b_{k, j}+U_{k, j}$. If $a_{0}<0$, then, by Fact 1, all $b_{k, j}<0$. Analogously, if $a_{0}>0$, then all $b_{k, j}>0$. It follows that all the $U_{k, j}, j=0, \ldots, k-1$, are different. Therefore, $\operatorname{sval}(M)=\infty$ in contradiction to our assumption.

Property (S2). $M$ has Property (S2') for proper $t \in T_{\Sigma}\left(x_{1}\right)$ iff, for every decomposition $t=t_{1}\left[x_{1}, t_{2} t_{3}\right]$ with proper $t_{1} \in T_{\Sigma}\left(X_{2}\right)$, proper $t_{2} \in T_{\Sigma}\left(x_{1}\right)$, and $t_{3} \in T_{\Sigma}$,

$$
\begin{aligned}
& \left(p, t_{1}, s_{11}, p p_{1}\right),\left(p_{1}, t_{2}, s_{12}, p_{1}\right),\left(p_{1}, t_{3}, s_{13}, \varepsilon\right), \\
& \left(p, t_{1}, s_{21}, q p_{2}\right),\left(p_{2}, t_{2}, s_{22}, p_{2}\right),\left(p_{2}, t_{3}, s_{23}, \varepsilon\right), \\
& \left(q, t_{1}, s_{31}, q p_{3}\right),\left(p_{3}, t_{2}, s_{32}, p_{3}\right),\left(p_{3}, t_{3}, s_{33}, \varepsilon\right) \in \delta
\end{aligned}
$$

with $\omega\left(s_{i j}\right)=g_{i j}$ implies

$$
g_{11}\left[x_{1}, g_{12} g_{13}\right]-g_{11}\left[x_{1}, g_{13}\right]=g_{21}\left[x_{1}, g_{22} g_{23}\right]-g_{21}\left[x_{1}, g_{23}\right] \text {. }
$$




\section{Fact 3.}

(i) Provided $q \notin \operatorname{Const}(M)$, (S2') is equivalent to

$$
g_{11}\left[x_{1}, g_{12} g_{13}\right] g_{11}\left[x_{1}, g_{13}\right]^{-1}=g_{21}\left[x_{1}, g_{22} g_{23}\right] g_{21}\left[x_{1}, g_{23}\right]^{-1} \text {. }
$$

(ii) Assume

$$
\begin{aligned}
& \omega\left(s_{i 1}\right)=a_{i}+e_{i} x_{1}+f_{i} x_{2} . \\
& \omega\left(s_{i 2}\right)=n_{i}+d_{i} x_{1}, \\
& \omega\left(s_{i 3}\right)=b_{i}, \quad i=1,2 .
\end{aligned}
$$

Then (S2') takes the form

$$
f_{1} n_{1}+\left(d_{1}-1\right) f_{1} b_{1}=f_{2} n_{2}+\left(d_{2}-1\right) f_{2} b_{2} \text {. }
$$

In proofs we mainly refer to the version of (S2') given under (ii). Especially note that this version does not depend on $a_{i}$ or $e_{i}$. Assertion (i) is used in Section 5 in the proof of the (technical) Proposition 5.3.

\section{Proposition 3.6.}

(i) Assume $t \in T_{\Sigma}\left(x_{1}\right)$ is proper, and $M$ has Property (S0) for $t_{1}\left[x_{1}, t_{3}\right]$ whenever $t=t_{1}\left[x_{1}, t_{2} t_{3}\right]$ for proper $t_{1} \in T_{\Sigma}\left(X_{2}\right)$, proper $t_{2} \in T_{\Sigma}\left(x_{1}\right)$, and $t_{3} \in T_{\Sigma}$. Then $M$ has Property (S2) for $t$ iff $M$ has Property (S2') for $t$.

(ii) If $\operatorname{sval}(M)<\infty$, then $M$ has Property (S2').

Proof. Assume $p, p_{i}, q, t_{i}$, and $s_{i j}$ are chosen according to the assumptions of Properties $\left(\mathrm{S} 2^{\prime}\right)$ and $(\mathrm{S} 2)$. We show that the conclusion according to $(\mathrm{S} 2)$ is equivalent to the conclusion according to $\left(\mathrm{S} 2^{\prime}\right)$.

Define $g_{i j}:=\omega\left(s_{i j}\right)$, and $g_{i 1}:=a_{i}+e_{i} x_{1}+f_{i} x_{2}, g_{i 2}=n_{i}+d_{i} x_{1}, g_{i 3}=b_{i}$ for $i=1,2,3$.

(i) Property (SO) for $t_{1}\left[x_{1}, t_{3}\right]$ implies

$$
a_{1}+e_{1}\left[a_{2}+e_{2} x_{1}+f_{2} b_{2}\right]+f_{1} b_{1}=a_{2}+e_{2}\left[a_{3}+e_{3} x_{1}+f_{3} b_{3}\right]+f_{2} b_{2} .
$$

The conclusion in (S2) is

$$
\begin{aligned}
a_{1}+ & e_{1}\left[a_{2}+e_{2} x_{1}+f_{2} b_{2}\right]+f_{1}\left[n_{1}+d_{1} b_{1}\right] \\
& =a_{2}+e_{2}\left[a_{3}+e_{3} x_{1}+f_{3} b_{3}\right]+f_{2}\left[n_{2}+d_{2} b_{2}\right] .
\end{aligned}
$$

Subtracting (1) from (2) yields

$$
f_{1}\left[n_{1}+\left(d_{1}-1\right) \cdot b_{1}\right]=f_{2}\left[n_{2}+\left(d_{2}-1\right) \cdot b_{2}\right],
$$

which is the conclusion of $\left(\mathrm{S}^{\prime}\right)$. 
(ii) Since $M$ is reduced, $t \in T_{\Sigma}$ and $s \in T_{\Delta} \cup\{\perp\}$ exist with $(q, t, s, \varepsilon) \in \delta$. Let $m:=\omega(s)$. For $j, k \geq 0$, define $t^{(j)}:=t_{1}\left[x_{1}, t_{2}^{j} t_{3}\right]$, and $\bar{t}^{(k)}:=t^{(k)} t^{(k-1)} \cdots t^{(0)}$. Define $v_{i}^{(j)}:=s_{i 1}\left[x_{1}, s_{i 2}^{j} s_{i 3}\right], i=1,2,3$, with $h_{i}^{(j)}:=g_{i 1}\left[x_{1}, g_{i 2}^{j} g_{i 3}\right]=\omega\left(v_{i}^{(j)}\right)$ and $u_{k, j}:=$ $v_{1}^{(k)} \cdots v_{1}^{(j+1)} v_{2}^{(j)} v_{3}^{(j-1)} \cdots v_{3}^{(0)}$ for $0 \leq j \leq k-1$. Then $\left(p, \bar{t}^{(k)}, u_{k, j}, q\right) \in \delta$ for all $j \in\{0, \ldots, k-1\}$.

First assume $q \in$ Const $(M)$. Then, by Proposition $2.4, s_{31}\left[x_{1}, s_{32} s_{33}\right] \in\left\{\perp, x_{1}\right\}$. Hence, by Proposition 3.2, $g_{11}\left[x_{1}, g_{12} g_{13}\right] \in\left\{0, x_{1}\right\}$. If $p \in U(M)$, then $g_{11}=$ $g_{12}=g_{21}=g_{22}=0$ by the reducedness of $M$, and the conclusion of (S2') trivially holds. Therefore, assume $p \notin U(M)$. Since $g_{11}\left[x_{1}, g_{12} g_{13}\right]=x_{1}, g_{12} g_{13}=0=g_{13}$ by the reducedness of $M$, and the left-hand side of (S2') equals 0 . Moreover,

$$
\omega\left(u_{k, j} s\right)=\omega\left(v_{2}^{(j)} s\right)=a_{2}+e_{2} m+f_{2} n_{2} \cdot \sum_{i=0}^{j-1} d_{2}^{i}+f_{2} b_{2} \cdot d_{2}^{j} .
$$

Consequently,

$$
\omega\left(u_{k, j+1} s\right)-\omega\left(u_{k, j} s\right)=f_{2} d_{2}^{j}\left[n_{2}+b_{2}\left(d_{2}-1\right)\right] \geq 0 .
$$

If this is different from 0 it follows that $\omega\left(u_{k, j} s\right)$ are distinct for all $j=0, \ldots, k-1$, which gives us a contradiction to $\operatorname{sval}(M)<\infty$. Therefore, $f_{2} d_{2}^{j}\left[n_{2}+b_{2}\left(d_{2}-1\right)\right]$ $=0$. If $f_{2} d_{2}^{j}=0$, then (by the reducedness of $\left.M\right) p_{2} \in U(M)$. Hence $f_{2}=0$, which implies that the right-hand side of (S2') equals 0 as well. If $f_{2} d_{2}^{j}>0$, then $n_{2}+b_{2}$. $\left(d_{2}-1\right)=0$, which again implies that the right-hand side of $\left(\mathrm{S} 2^{\prime}\right)$ equals 0 . Hence, the conclusion of Property (S2') holds.

Now assume $q \notin \operatorname{Const}(M)$. Then all $h_{i}^{(j)}$ are invertible. Applying Proposition 3.2 to $t^{(j)}$ we obtain

$$
h_{1}^{(j)} h_{2}^{(j)}=h_{2}^{(j)} h_{3}^{(j)}
$$

Moreover, we have

$$
\begin{aligned}
\omega\left(u_{k, j+1}\right) & =h_{1}^{(k)} \cdots h_{1}^{(j+2)} h_{2}^{(j+1)} h_{3}^{(j)} \cdots h_{3}^{(0)} \\
& =h_{1}^{(k)} \cdots h_{1}^{(j+2)} A^{(j)} h_{1}^{(j+1)} h_{2}^{(j)} h_{3}^{(j-1)} \cdots h_{3}^{(0)} \\
& =\left(B_{k, j} A^{(j)} B_{k, j}^{-1}\right) \omega\left(u_{k, j}\right)
\end{aligned}
$$

for some invertible $B_{k, j} \in \mathbb{N}_{0}^{(1)}\left[x_{1}\right]$ where

$$
\begin{aligned}
A^{(j)} & =h_{2}^{(j+1)} h_{3}^{(j)}\left[h_{2}^{(j)}\right]^{-1}\left[h_{1}^{(j+1)}\right]^{-1} \\
& =h_{2}^{(j+1)}\left[h_{2}^{(j)}\right]^{-1} h_{1}^{(j)}\left[h_{1}^{(j+1)}\right]^{-1} \quad \text { by (1). }
\end{aligned}
$$

First we show, by induction on $j$,

$$
g_{i 2}^{j+1} g_{i 3}-g_{i 2}^{j} g_{i 3}=d_{i}^{j} \cdot\left[g_{i 2} g_{i 3}-g_{i 3}\right] \text {. }
$$

By definition, (3) holds for $j=0$. Assume $j>0$, and (3) holds for $j-1$. Then

$$
\begin{aligned}
g_{i 2}^{j+1} g_{i 3}-g_{i 2}^{j} g_{i 3} & =n_{i}+d_{i} \cdot g_{i 2}^{j} g_{i 3}-n_{i}-d_{i} \cdot g_{i 2}^{j-1} g_{i 3} \\
& =d_{i} \cdot\left[g_{i 2}^{j} g_{i 3}-g_{i 2}^{j-1} g_{i 3}\right] \\
& =d_{i} \cdot d_{i}^{j-1} \cdot\left[g_{i 2} g_{i 3}-g_{i 3}\right] \quad \text { by the induction hypothesis, }
\end{aligned}
$$

which proves (3). 
We conclude that

$$
\begin{aligned}
h_{i}^{(j+1)}\left[h_{i}^{(j)}\right]^{-1} & =g_{i 1}\left[x_{1}, g_{i 2}^{j+1} g_{i 3}\right] g_{i 1}\left[x_{1}, g_{i 2}^{j} g_{i 3}\right]^{-1} \\
& =f_{i} \cdot\left[g_{i 2}^{j+1} g_{i 3}-g_{i 2}^{j} g_{i 3}\right]+x_{1} \\
& =f_{i} \cdot d_{i}^{j} \cdot\left[g_{i 2} g_{i 3}-g_{i 3}\right]+x_{1} \quad \text { by (3). }
\end{aligned}
$$

Hence,

$$
A^{(j)}=a^{(j)}+x_{1}
$$

where

$$
a^{(j)}=f_{2} \cdot d_{2}^{j} \cdot\left[g_{22} g_{23}-g_{23}\right]-f_{1} \cdot d_{1}^{j} \cdot\left[g_{12} g_{13}-g_{13}\right] .
$$

Using (5) and Fact 1, (2) can be rewritten as

$$
\omega\left(u_{k, j+1}\right)=b_{k, j} \cdot a^{(j)}+\omega\left(u_{k, j+1}\right)
$$

for some $b_{k, j} \in \mathbb{N}$. We distinguish between the different possibilities for $d_{i}, i=1,2$.

Case 1: $d_{1}=0$. Then $p_{1} \in U(M)$. Hence, $f_{1}=0$ and, therefore,

$$
f_{1} \cdot\left[g_{12} g_{13}-g_{13}\right]=0 \text { and } a^{(j)}=f_{2} \cdot d_{2}^{j} \cdot\left[g_{22} g_{23}-g_{23}\right] \text {. }
$$

If $a^{(j)}=0$, then the conclusion of (S2') is satisfied. Therefore, assume $a^{(j)} \neq 0$. If $a^{(j)}>0$, then $\omega\left(u_{k, j+1}\right)-\omega\left(u_{k, j}\right)>0$ for all $j$. Analogously, if $a^{(j)}<0$, then $\omega\left(u_{k, j+1}\right)-\omega\left(u_{k, j}\right)<0$ for all $j$. In both cases all the output sizes $\omega\left(u_{k, j}\right)$, $j=0, \ldots, k-1$, are distinct. Since $q \notin \operatorname{Const}(M)$ this gives a contradiction to $\operatorname{sval}(M)<\infty$.

Case 2: $d_{2}=0$. This case is analogous to Case 1 with the role of $d_{1}$ and $d_{2}$ exchanged.

Case 3: $d_{1} \neq 0 \neq d_{2}$. We consider three subcases.

Case 3.1: $d_{1}=d_{2}=: d$. Then

$$
a^{(j)}=d^{j} \cdot\left[f_{2} \cdot\left(g_{22} g_{23}-g_{23}\right)-f_{1} \cdot\left(g_{12} g_{13}-g_{13}\right)\right] .
$$

If $a^{(j)}=0$, then $f_{2} \cdot\left(g_{22} g_{23}-g_{23}\right)-f_{1} \cdot\left(g_{12} g_{13}-g_{13}\right)=0$, and the conclusion of $\left(\mathbf{S} 2^{\prime}\right)$ is satisfied, whereas $a^{(j)} \neq 0$ gives a contradiction as in Case 1.

Case 3.2: $d_{2}>d_{1}>0$. Especially, $d_{2}>1$. Define $c:=d_{1} / d_{2}$. Then

$$
a^{(j)}=d_{2}^{j} \cdot\left[f_{2} \cdot\left(g_{22} g_{23}-g_{23}\right)-c^{j} \cdot f_{1} \cdot\left(g_{12} g_{13}-g_{13}\right)\right] \text {. }
$$

Since $0<c<1$, some $j_{0} \in \mathbb{N}$ exists such that, for all $j \geq j_{0}$,

$$
\left.\mid c^{j} \cdot f_{1} \cdot\left(g_{12} g_{13}-g_{13}\right)\right] \mid<1 \text {. }
$$

Assume $g_{22} g_{23}-g_{23}=0$. Then $g_{22} \in\left\{0, x_{1}\right\}$, which is impossible since $d_{2}>1$. Therefore, $g_{22} g_{23}-g_{23} \geq 1$. It follows that $a^{(j)}>0$ at least for all $j \geq j_{0}$. Hence, for all $k>j_{0}, \omega\left(u_{k, j+1}\right)-\omega\left(u_{k, j}\right)>0$ whenever $j_{0} \leq j<k$. Therefore, the output 
sizes $\omega\left(u_{k, j}\right), j \in\left\{j_{0}, \ldots, k\right\}$, are distinct. Since we can choose $k$ arbitrarily large, this gives a contradiction to $\operatorname{sval}(M)<\infty$.

Case 3.3: $d_{1}>d_{2}>0$. Here the argumentation is analogous to Case 3.2 where we considered $d_{2}>d_{1}$. We derive that some $j_{1} \in \mathbb{N}$ exist such that, for all $k>j_{1}$, $\omega\left(u_{k, j+1}\right)-\omega\left(u_{k, j}\right)<0$ for $j_{1} \leq j<k$; which again gives a contradiction to $\operatorname{sval}(M)<\infty$. This finishes the proof.

Proof of Theorem 3.1. Assume sval $(M)<\infty$. By Proposition 3.2, $M$ has Property (S0). By Propositions 3.5(ii) and 3.6(ii), $M$ has Properties (S1') and (S2'). Therefore, by Propositions 3.5(i) and 3.6(i), $M$ has Propoerty (S1) and (S2) as well. This proves statement (i).

Now assume $\operatorname{val}(M)<\infty$. Especially, $\operatorname{sval}(M)<\infty$. Therefore, by statement (i), $M$ has Properties (S1) and (S2). Since by Proposition 3.3 $M$ also has Property (F0'), we conclude with Proposition 3.4 that $M$ has Properties (F1) and (F2) as well. This proves (ii).

\section{Decidability}

The main theorem of this section (Theorem 4.1) states that it can be decided whether or not a reduced FST $M$ has Properties (S1) and (S2) or Properties (F1) and $(\mathrm{F} 2)$ and gives upper complexity bounds. In order to prove this result we show that $M$ has the corresponding pair of properties provided $M$ has these properties for all trees up to a depth polynomial in the size of $M$ (Proposition 4.2).

Theorem 4.1. Assume $M$ is a reduced FST.

(i) It can be decided in polynomial space whether or not $M$ has Properties (S1) and (S2).

(ii) It can be decided in nondeterministic polynomial time whether $M$ does not have Properties (F1) and (F2).

Assume $n=\# Q$ is the number of states of $M$. The crucial step in proving Theorem 4.1 is given by the following proposition:

\section{Proposition 4.2.}

(i) If $M$ has Property $\left(\mathrm{S}^{\prime}\right)$ for all proper $t \in T_{\Sigma}\left(x_{1}\right)$ up to depth $4 n^{3}$ and Property (S2'), then $M$ has Property (S1').

(ii) If $M$ has Property (S2') for all proper $t \in T_{\Sigma}\left(x_{1}\right)$ up to depth $6 n^{3}$, then $M$ has Property (S2').

(iii) Assume $M$ has Properties (S1') and (S2'). If $M$ has Property (F0') for all proper trees $t \in T_{\Sigma}\left(x_{1}\right)$ up to depth $6 n^{4}$, then $M$ has Property (F0'). 
Proof of Theorem 4.1. Assume $M$ has Properties (S1) and (S2) for trees up to depth $6 n^{3}$. Then, especially, $M$ has Property (S0) for all trees up to depth $6 n^{3}$. Hence, by Propositions 3.5(i) and 3.6(i), $M$ has Properties ( $\left(\mathrm{S}^{\prime}\right)$ and ( $\left.\mathbf{S 2}^{\prime}\right)$ for all trees up to depth $6 n^{3}$. Applying first statement (ii) and then statement (i) of Proposition 4.2, we deduce that $M$ has Properties (S1) and (S2') and also (by Proposition 3.5(i) again) Property (S0). Consequently, by Propositions 3.5(i) and 3.6(i) (now in reverse direction), $M$ has Properties (S1) and (S2). This proves statement (i) of Theorem 4.1.

Assume $M$ additionally has Property (F0) for all trees up to depth $6 n^{4}$. By Proposition 3.4, $M$ also has Property (F0') for all trees up to depth $6 n^{4}$. Therefore, by Propositions 4.2(iii), $M$ has Property (F0'), and hence, by Proposition 3.4(ii), Properties (Fi) where $i=0,1,2$. We conclude: if $M$ has Properties (F1) and (F2) for all trees up to depth $6 n^{4}$, then $M$ has Properties (F1) and (F2). (ii) follows from this.

Proof of Proposition 4.2. (i) For a contradiction, assume the proper tree $t \in T_{\Sigma}\left(x_{1}\right)$ is of minimal $\Sigma$-size such that $t=t_{1} t_{2} t_{3}$ for proper trees $t_{i}$ and

$$
\begin{aligned}
& \left(p, t_{1}, s_{11}, p_{1}\right),\left(p_{1}, t_{2}, s_{12}, p_{1}\right),\left(p_{1}, t_{3}, s_{13}, p\right), \\
& \left(p, t_{1}, s_{21}, p_{2}\right),\left(p_{2}, t_{2}, s_{22}, p_{2}\right),\left(p_{2}, t_{3}, s_{23}, q\right), \\
& \left(q, t_{1}, s_{31}, p_{3}\right),\left(p_{3}, t_{2}, s_{32}, p_{3}\right),\left(p_{3}, t_{3}, s_{33}, q\right) \in \delta
\end{aligned}
$$

for certain states $p, p_{i}, q \in Q$ and output trees $s_{i v} \in T_{\Delta}\left(x_{1}\right)$ such that the conclusion of $\left(\mathrm{S}^{\prime}\right)$ does not hold. Moreover, assume that the decomposition of $t$ is chosen such that $t_{2}$ is of minimal $\Sigma$-size. By assumption, $\operatorname{depth}(t)>4 n^{3}$.

First assume $q \in \operatorname{Const}(M)$. In this case the conclusion of $\left(\mathrm{Si}^{\prime}\right)$ is $\omega\left(s_{12}\right)$, $\omega\left(s_{22}\right) \in\left\{0, x_{1}\right\}$, which is independent of $s_{i 1}$ and $s_{i 3}$. Hence, the conclusion holds for $t=t_{1} t_{2} t_{3}$ provided it holds for $t_{1}^{\prime} t_{2} t_{3}^{\prime}$ where $t_{1}^{\prime}$ and $t_{3}^{\prime}$ are fixed proper input trees for which $\left(p, p_{1}\right)-,\left(p, p_{2}\right)-,\left(q, p_{3}\right)$-computations and $\left(p_{1}, p\right)-,\left(p_{2}, q\right)$-, and $\left(p_{3}, q\right)$-computations respectively exist. If these computations exist, then the conclusion also holds for trees $t_{1}^{\prime}$ and $t_{3}^{\prime}$ of depth at most $2 n^{3}$ where the paths to the leafs labeled $x_{1}$ have length at most $n^{3}$.

Hence if $\operatorname{depth}(t)>4 n^{3}$, one of the following two cases must occur.

Case 1: The path in $t_{2}$ to the leaf $x_{1}$ has length $>n^{3}$. Hence, $t_{2}=u_{1} u_{2} u_{3}$ for proper $u_{v} \in T_{\Sigma}\left(x_{1}\right)$ with $u_{2} \neq x_{1} \neq u_{1} u_{3}$ such that

$$
\left(p_{i}, u_{1}, r_{i 1}, q_{i}\right),\left(q_{i}, u_{2}, r_{i 2}, q_{i}\right),\left(q_{i}, u_{3}, r_{i 3}, p_{i}\right) \in \delta, \quad i=1,2,3,
$$

for some states $q_{i}$ and decompositions $s_{i 2}=r_{i 1} r_{i 2} r_{i 3}$. The $\Sigma$-sizes of both $u_{2}$ and $u_{1} u_{3}$ are strictly less than $\left|t_{2}\right|_{\Sigma}$. The latter implies that $M$ has Property $\left(\mathrm{S} 1^{\prime}\right)$ for $t^{\prime}:=t_{1} u_{1} u_{3} t_{3}$. Hence, $\omega\left(r_{i 1} r_{i 3}\right) \in\left\{0, x_{1}\right\}$ and therefore both $\omega\left(r_{i 1}\right)$ and $\omega\left(r_{i 3}\right) \in\left\{0, x_{1}\right\}$ for $i=1,2$. By minimality of $t_{2}$ in the decomposition of $t$, the conclusion (S1') holds for $t=t_{1}^{\prime} t_{2}^{\prime} t_{3}^{\prime}$ where $t_{1}^{\prime}:=t_{1} u_{1}, t_{2}^{\prime}:=u_{2}$, and $t_{3}^{\prime}:=u_{3} t_{3}$. We conclude that $\omega\left(r_{i 2}\right) \in\left\{0, x_{1}\right\}$ for $i=1,2$ as well. Together, we find $\omega\left(s_{i 2}\right)=\omega\left(r_{i 1} r_{i 2} r_{i 3}\right) \in\left\{0, x_{1}\right\}$ for $i=1,2$, in contradiction to our assumption. 
Case 2: $t_{2}$ contains a subtree from $T_{\Sigma}$ of depth $>n^{3}$. Then $t_{2}=u_{1}\left[x_{1}, u_{2} u_{3}\right]$ for proper $u_{i}$ where $u_{2} \neq x_{1}$ such that

$$
\left(p_{i}, u_{1}, r_{i 1}, p_{i} q_{i}\right),\left(q_{i}, u_{2}, r_{i 2}, q_{i}\right),\left(q_{i}, u_{3}, r_{i 3}, \varepsilon\right) \in \delta, \quad i=1,2,3,
$$

for certain states $q_{i}$ and decompositions $s_{i 2}=r_{i 1}\left[x_{1}, r_{i 2} r_{i 3}\right]$. Since $\left|u_{1}\left[x_{1}, u_{3}\right]\right|_{\Sigma}<$ $\left|t_{2}\right|_{\Sigma}$, minimality of $t$ implies that Property $\left(\mathrm{S} 1^{\prime}\right)$ holds for $t^{\prime}=t_{1} u_{1}\left[t_{3}, u_{3}\right]$. Whence, $\omega\left(r_{i 1}\left[x_{1}, r_{i 3}\right]\right) \in\left\{0, x_{1}\right\}, i=1,2$. This means that $x_{2}$ does not occur in $\omega\left(r_{11}\right)$ or in $\omega\left(r_{21}\right)$. It follows that $\omega\left(r_{i 1}\left[x_{1}, r_{i 2} r_{i 3}\right]\right)=\omega\left(\mathrm{r}_{i 1}\left[x_{1}, r_{i 3}\right]\right)$. Therefore, the conclusion (S1') for $t$ holds as well, contradicting our assumption.

Thus, the conclusion of Property ( $\left(\mathbf{S}^{\prime}\right)$ holds for every $t$ provided $q \in \operatorname{Const}(M)$. It remains to consider $q \notin \operatorname{Const}(M)$. Assume the sizes of the outputs $s_{i j}$ in the assumption of Property (S1') are given by

$$
\begin{aligned}
& \omega\left(s_{i 1}\right)=a_{i}+e_{i} \cdot x_{1}, \\
& \omega\left(s_{i 2}\right)=n_{i}+d_{i} \cdot x_{1}, \quad i=1,2 .
\end{aligned}
$$

Since the assertions of Property ( $\left.\mathrm{S} 1^{\prime}\right)$ are independent of $t_{3}$, a similar argumentation as above shows that without loss of generality we may assume that depth $\left(t_{3}\right) \leq 2 n^{3}$ and the path in $t_{3}$ to leaf $x_{1}$ has length at most $n^{3}$. The remainder of the proof is a case analysis according to different possibilities for $t_{1}$ and $t_{2}$ in the factorization of $t$.

Case 1: $t_{1}$ is too "large."

Case 1.1: The path in $t_{1}$ to the leaf $x_{1}$ has length $>n^{3}$. Then $t_{1}=u_{1} u_{2} u_{3}$ for proper trees $u_{v}$ where $u_{2} \neq x_{1} \neq u_{1} u_{3}$ such that

$$
\begin{aligned}
& \left(p, u_{1}, r_{11}, q_{1}\right),\left(q_{1}, u_{2}, r_{12}, q_{1}\right),\left(q_{1}, u_{3}, r_{13}, p_{1}\right), \\
& \left(p, u_{1}, r_{21}, q_{2}\right),\left(q_{2}, u_{2}, r_{22}, q_{2}\right),\left(q_{2}, u_{3}, r_{23}, p_{2}\right), \\
& \left(q, u_{1}, r_{31}, q_{3}\right),\left(q_{3}, u_{2}, r_{32}, q_{3}\right),\left(q_{3}, u_{3}, r_{33}, p_{3}\right) \in \delta
\end{aligned}
$$

for certain states $q_{i}$ and decompositions $s_{i 1}=r_{i 1} r_{i 2} r_{i 3}$ where $\omega\left(r_{i v}\right)=a_{i v}+e_{i v} \cdot x_{1}$.

Consider the trees $t^{\prime}:=u_{1} u_{3} t_{2} t_{3}$ and $t^{\prime \prime}:=u_{1} u_{2} u_{3} t_{3}$. Since $u_{2} \neq x_{1},\left|t^{\prime}\right|_{\Sigma}<|t|_{\Sigma}$. The conclusion (S1') trivially holds if $t_{2}=x_{1}$. Therefore, $t_{2} \neq x_{1}$ and hence $\left|t^{\prime \prime}\right|_{\Sigma}<|t|_{\Sigma}$ as well. Whence, by minimality of $t, M$ has Property (S1') both for $t^{\prime}=\left(u_{1} u_{3}\right) t_{2} t_{3}$ and $t^{\prime \prime}=u_{1} u_{2}\left(u_{3} t_{3}\right)$. This gives us the following four equations (see Fact 2(ii)):

$$
\begin{aligned}
& d_{1}=d_{2}, \\
& \left(d_{1}-1\right)\left[a_{21}+e_{21} a_{23}-a_{11}-e_{11} a_{13}\right]=e_{21} e_{23} n_{2}-e_{11} e_{13} n_{1}, \\
& e_{12}=e_{22}, \\
& \left(e_{12}-1\right)\left[a_{21}-a_{11}\right]=e_{21} a_{22}-e_{11} a_{12} \ldots
\end{aligned}
$$


Therefore,

$$
\begin{aligned}
& \left(d_{1}-1\right) \cdot\left(a_{2}-a_{1}\right)-\left[e_{2} n_{2}-e_{1} n_{1}\right] \\
& =\left(d_{1}-1\right)\left[a_{21}+e_{21} a_{22}+e_{21} e_{22} a_{23}-a_{11}-e_{11} a_{12}-e_{11} e_{12} a_{13}\right] \\
& \quad-\left[e_{21} e_{22} e_{23} n_{2}-e_{11} e_{12} e_{13} n_{1}\right] \\
& \quad \stackrel{(4)}{=}\left(d_{1}-1\right)\left[e_{12} a_{21}+e_{21} e_{22} a_{23}-e_{12} a_{11}-e_{11} e_{12} a_{13}\right] \\
& \quad-\left[e_{21} e_{22} e_{23} n_{2}-e_{11} e_{12} e_{13} n_{1}\right] \\
& \quad \stackrel{(3)}{=} e_{12}\left(d_{1}-1\right)\left[a_{21}+e_{21} a_{23}-a_{11}-e_{11} a_{13}\right]-e_{12}\left[e_{21} e_{23} n_{2}-e_{11} e_{13} n_{1}\right] \\
& \quad \stackrel{(2)}{=} 0 .
\end{aligned}
$$

Hence, $M$ has Property (S1') for $t$, contradicting our assumption.

Case 1.2: $t_{1}$ contains a subtree from $T_{\Sigma}$ of depth $>n^{3}$. Then $t_{1}=u_{1}\left[x_{1}, u_{2} u_{3}\right]$ for proper trees $u_{v}$ where $u_{2} \neq x_{1}$ such that

$$
\begin{aligned}
& \left(p, u_{1}, r_{11}, p_{1}, q_{1}\right),\left(p, u_{2}, r_{12}, q_{1}\right),\left(q, u_{3}, r_{13}, \varepsilon\right), \\
& \left(p, u_{1}, r_{21}, p_{2} q_{2}\right),\left(q_{2}, u_{2}, r_{22}, q_{2}\right),\left(q_{2}, u_{3}, r_{23}, \varepsilon\right), \\
& \left(q, u_{1}, r_{31}, p_{3} q_{3}\right),\left(q_{3}, u_{2}, r_{32}, q_{3}\right),\left(q_{3}, u_{3}, r_{33}, \varepsilon\right) \in \delta
\end{aligned}
$$

for certain states $q_{i}$ and factorizations $s_{i 1}=r_{i 1}\left[x_{1}, r_{i 2} r_{i 3}\right]$ with

$$
\begin{aligned}
& \omega\left(r_{i 1}\right)=a_{i 1}+e_{i} \cdot x_{1}+f_{i 1} \cdot x_{2}, \\
& \omega\left(r_{i 2}\right)=a_{i 2}+d_{i}^{\prime} \cdot x_{1}, \\
& \omega\left(r_{i 3}\right)=a_{i 3} \quad \text { for } \quad i=1,2 .
\end{aligned}
$$

By minimality of $t, M$ has Property $\left(\mathrm{S}^{\prime}\right)$ for $t^{\prime}:=u_{1}\left[t_{2} t_{3}, u_{3}\right]$. This gives us

$$
\begin{aligned}
& d_{1}=d_{2}, \\
& \left(d_{1}-1\right)\left[a_{21}+f_{21} a_{23}-a_{11}-f_{11} a_{13}\right]=e_{2} n_{2}-e_{1} n_{1} .
\end{aligned}
$$

Moreover, by assumption, $M$ has Property (S2') for $t^{\prime \prime}:=u_{1}\left[t_{3}, u_{2} u_{3}\right]$ with factors $u_{1}\left[t_{3}, x_{2}\right], u_{2}$, and $u_{3}$. We deduce

$$
f_{11} a_{12}+\left(d_{1}^{\prime}-1\right) f_{11} a_{13}=f_{21} a_{22}+\left(d_{2}^{\prime}-1\right) f_{21} a_{23} .
$$

It follows that

$$
\begin{aligned}
& \left(d_{1}-1\right) \cdot\left(a_{2}-a_{1}\right) \\
& \quad=\left(d_{1}-1\right)\left[a_{21}+f_{21} a_{22}+f_{21} d_{2}^{\prime} a_{23}-a_{11}-f_{11} a_{12}-f_{11} d_{1}^{\prime} a_{13}\right] \\
& \quad \stackrel{(3)}{=}\left(d_{1}-1\right)\left[a_{21}+f_{21} a_{23}-a_{11}-f_{11} a_{13}\right] \\
& \quad \stackrel{(2)}{=} e_{2} n_{2}-e_{1} n_{1} .
\end{aligned}
$$

Hence, $M$ has Property $\left(\mathrm{S}^{\prime}\right)$ for $t$, in contradiction to our assumption. 
Case 2: $t_{2}$ is too "large."

Case 2.1: The path in $t_{2}$ to the leaf $x_{1}$ has length $>n^{3}$. Then $t_{2}=u_{1} u_{2} u_{3}$ for proper trees $u_{v}$ with $u_{2} \neq x_{1}$ such that

$$
\left(p_{i}, u_{1}, r_{i 1}, q_{i}\right),\left(q_{i}, u_{2}, r_{i 2}, q_{i}\right),\left(q_{i}, u_{3}, r_{i 3}, p_{i}\right) \in \delta, \quad \text { for } \quad i=1,2,3,
$$

for certain states $q_{i}$ and decompositions $s_{i 2}=r_{i 1} r_{i 2} r_{i 3}$ where $\omega\left(r_{i v}\right)=n_{i v}+d_{i v} \cdot x_{1}$.

By minimality of $t, M$ has Property (S1') for $t^{\prime}:=t_{1} u_{1} u_{3} t_{3}$. By minimality of $t_{2}, M$ allows for the conclusion (S1') for the factorization $t=t_{1}^{\prime} t_{2}^{\prime} t_{3}^{\prime}$ where $t_{1}^{\prime}:=t_{1} u_{1}, t_{2}^{\prime}:=u_{2}$, and $t_{3}^{\prime}:=u_{3} t_{3}$. Therefore,

$$
\begin{aligned}
& d_{11} d_{13}=d_{21} d_{23}, \\
& \left(d_{11} d_{13}-1\right)\left[a_{2}-a_{1}\right]=e_{2}\left[n_{21}+d_{21} n_{23}\right]-e_{1}\left[n_{11}+d_{11} n_{13}\right] \\
& d_{12}=d_{22}, \\
& \left(d_{12}-1\right)\left[a_{2}+e_{2} n_{21}-a_{1}-e_{1} n_{11}\right]=e_{2} d_{21} n_{22}-e_{1} d_{11} n_{12} .
\end{aligned}
$$

From (1) and (3) we obtain

$$
d_{1}=d_{11} d_{12} d_{13}=d_{21} d_{22} d_{23}=d_{2} .
$$

Moreover,

$$
\begin{aligned}
\left(d_{1}-\right. & 1) \cdot\left(a_{2}-a_{1}\right)-\left[e_{2} n_{2}-e_{1} n_{1}\right] \\
= & \left(d_{11} d_{12} d_{13}-1\right)\left[a_{2}-a_{1}\right]-e_{2}\left[n_{21}+d_{21} n_{22}+d_{21} d_{22} n_{23}\right] \\
& +e_{1}\left[n_{11}+d_{11} n_{12}+d_{11} d_{12} n_{13}\right] \\
& \stackrel{(2)}{=} \\
\quad & \left(d_{12}-1\right)\left[a_{2}-a_{1}\right]-e_{2}\left[\left(1-d_{12}\right) n_{21}+d_{21} n_{22}+d_{21}\left(d_{22}-d_{12}\right) n_{23}\right] \\
& +e_{1}\left[\left(1-d_{12}\right) n_{11}+d_{11} n_{12}\right] \\
& \stackrel{(3)}{=} \\
& \left(d_{12}-1\right)\left[a_{2}-a_{1}\right]-e_{2}\left[\left(1-d_{12}\right) n_{21}+d_{21} n_{22}\right] \\
& +e_{1}\left[\left(1-d_{12}\right) n_{11}+d_{11} n_{12}\right] \\
& \stackrel{(4)}{=} 0 .
\end{aligned}
$$

Hence, $M$ allows for the conclusion (S1') also for the factorization $t=t_{1} t_{2} t_{3}$, in contradiction to our assumption.

Case 2.2: $t_{2}$ contains a subtree from $T_{\Sigma}$ of depth $>n^{3}$. Then $t_{2}=u_{1}\left[x_{1}, u_{2} u_{3}\right]$ for proper $u_{v}$ where $u_{2} \neq x_{1}$ such that

$$
\left(p_{i}, u_{1}, r_{i 1}, p_{i} q_{i}\right),\left(q_{i}, u_{2}, r_{i 2}, q_{i}\right),\left(q_{i}, u_{3}, r_{i 3}, \varepsilon\right) \in \delta \quad \text { for } \quad i=1,2,3
$$

for certain states $q_{i}$ and decompositions $s_{i 2}=r_{i 1}\left[x_{1}, r_{i 2} r_{i 3}\right]$ where

$$
\begin{aligned}
& \omega\left(r_{i 1}\right)=n_{i 1}+d_{i} \cdot x_{1}+f_{i} \cdot x_{2}, \\
& \omega\left(r_{i 2}\right)=n_{i 2}+d_{i 2} \cdot x_{1}, \\
& \omega\left(r_{i 3}\right)=n_{i 3} \quad \text { for } \quad i=1,2 .
\end{aligned}
$$


By minimality of $t, M$ has Property $\left(\mathrm{S} 1^{\prime}\right)$ for $t^{\prime}:=t_{1} u_{1}\left[t_{3}, u_{3}\right]$. Therefore,

$$
\begin{aligned}
& d_{1}=d_{2}, \\
& \left(d_{1}-1\right)\left[a_{2}-a_{1}\right]=e_{2}\left[n_{21}+f_{2} n_{23}\right]-e_{1}\left[n_{11}+f_{1} n_{13}\right]
\end{aligned}
$$

By assumption, $M$ has Property $\left(\mathrm{S} 2^{\prime}\right)$ for $t=t_{1}^{\prime}\left[x_{1}, t_{2}^{\prime} t_{3}^{\prime}\right]$ where $t_{1}^{\prime}:=t_{1} u_{1}\left[t_{3}, x_{2}\right]$, $t_{2}^{\prime}:=u_{2}$, and $t_{3}^{\prime}:=u_{3}$. This gives us

$$
e_{1} f_{1} n_{12}+e_{1} f_{1}\left(d_{12}-1\right) n_{13}=e_{2} f_{2} n_{22}+e_{2} f_{2}\left(d_{22}-1\right) n_{23} \text {. }
$$

Therefore,

$$
\begin{aligned}
e_{2} n_{2}-e_{1} n_{1} & =e_{2}\left[n_{21}+f_{2} n_{22}+f_{2} d_{22} n_{23}\right]-e_{1}\left[n_{11}+f_{1} n_{12}+f_{1} d_{12} n_{13}\right] \\
& \stackrel{(3)}{=} e_{2}\left[n_{21}+f_{2} n_{23}\right]-e_{1}\left[n_{11}+f_{1} n_{13}\right] \\
& \stackrel{(2)}{=}\left(d_{1}-1\right)\left[a_{2}-a_{1}\right] .
\end{aligned}
$$

Together with (1), we deduce that $M$ has Property (S1') also for $t$, in contradiction to our assumption.

(ii) Assume $M$ has Property (S2') for all proper trees up to depth $6 n^{3}$ but not for all trees. Then there is a proper tree $t=t_{1}\left[x_{1}, t_{2} t_{3}\right]$ of minimal $\Sigma$-size, states $p, q, p_{i}$, and $s_{i j}$ as in the assumption of Property $\left(\mathrm{S} 2^{\prime}\right)$ with

$$
\begin{aligned}
& \omega\left(s_{i 1}\right)=a_{i}+e_{i} \cdot x_{1}+f_{i} \cdot x_{2}, \\
& \omega\left(s_{i 2}\right)=n_{i}+d_{i} \cdot x_{1}, \\
& \omega\left(s_{i 3}\right)=b_{i}, \quad i=1,2,
\end{aligned}
$$

such that the conclusion of $\left(\mathrm{S2}^{\prime}\right)$ does not hold, i.e.,

$$
f_{1} \cdot\left[n_{1}+\left(d_{1}-1\right) \cdot b_{1}\right] \neq f_{2} \cdot\left[n_{2}+\left(d_{2}-1\right) \cdot b_{2}\right] \text {. }
$$

Moreover, assume that the factorization $t=t_{1}\left[x_{1}, t_{2} t_{3}\right]$ of $t$ is chosen such that $t_{2}$ is of minimal $\Sigma$-size. By assumption, depth $(t)>6 n^{3}$. We have to distinguish between the following cases:

Case 1: $t_{1}$ is too "large."

Case 1.1: $t_{1}$ contains a subtree from $T_{\Sigma}$ of depth $>n^{3}$. Then $t_{1}=u_{1}\left[x_{1}, x_{2}, u_{2} u_{3}\right]$ for proper $u_{1} \in T_{\Sigma}\left(X_{3}\right)$, proper $u_{2} \in T_{\Sigma}\left(x_{1}\right)$, and $u_{3} \in T_{\Sigma}$ with $u_{2} \neq x_{1}$ such that

$$
\begin{aligned}
& \left(p, u_{1}, r_{11}, p p_{1} q_{1}\right),\left(q_{1}, u_{2}, r_{12}, q_{1}\right),\left(q_{1}, u_{3}, r_{13}, \varepsilon\right), \\
& \left(p, u_{1}, r_{21}, q p_{2} q_{2}\right),\left(q_{2}, u_{2}, r_{22}, q_{2}\right),\left(q_{2}, u_{3}, r_{23}, \varepsilon\right), \\
& \left(q, u_{1}, r_{31}, q p_{3} q_{3}\right),\left(q_{3}, u_{2}, r_{32}, q_{3}\right),\left(q_{3}, u_{3}, r_{33}, \varepsilon\right) \in \delta
\end{aligned}
$$

for certain states $q_{i}$ and decompositions $s_{i 1}=r_{i 1}\left[x_{1}, x_{2}, r_{i 2} r_{i 3}\right]$.

Consider $t^{\prime}:=u_{1}\left[x_{1}, t_{2} t_{3}, u_{3}\right]$. Since $\left|t^{\prime}\right|_{\Sigma}<|t|_{\Sigma}, M$ has Property (S2') for $t^{\prime}$. $\omega\left(r_{i 1}\left[x_{1}, x_{2}, r_{i 3}\right]\right)$ only differs from $\omega\left(s_{i 1}\right)$ in the constant. Since this constant does not occur in the conclusion (S2'), the conclusion holds for $t$ as well, in contradiction to our assumption. 
Case 1.2: The common prefix of the paths in $t_{1}$ to $x_{1}$ and $x_{2}$ has length $>2 n^{3}$. Then $t_{1}=u_{1} u_{2} u_{3} u_{4}\left[x_{1}, x_{2}\right]$ for proper $u_{1}, u_{2}, u_{3} \in T_{\Sigma}\left(x_{1}\right)$ and proper $u_{4} \in T_{\Sigma}\left(X_{2}\right)$ with $u_{2}, u_{3} \neq x_{1}$ such that

$$
\begin{aligned}
& \left(p, u_{1}, r_{11}, q_{1}\right),\left(q_{1}, u_{2}, r_{12}, q_{1}\right),\left(q_{1}, u_{3}, r_{13}, q_{1}\right),\left(q_{1}, u_{4}, r_{14}, p p_{1}\right), \\
& \left(p, u_{1}, r_{21}, q_{2}\right),\left(q_{2}, u_{2}, r_{22}, q_{2}\right),\left(q_{2}, u_{3}, r_{23}, q_{2}\right),\left(q_{2}, u_{4}, r_{24}, q p_{2}\right), \\
& \left(q, u_{1}, r_{31}, q_{3}\right),\left(q_{3}, u_{2}, r_{32}, q_{3}\right),\left(q_{3}, u_{3}, r_{33}, q_{3}\right),\left(q_{3}, u_{4}, r_{34}, q p_{3}\right) \in \delta
\end{aligned}
$$

for certain states $q_{i}$ and decompositions $s_{i 1}=r_{i 1} r_{i 2} r_{i 3} r_{i 4}$.

Assume $\omega\left(r_{i v}\right)=a_{i v}+f_{i v} \cdot x_{1}, v=1,2,3$, and $\omega\left(r_{i 4}\right)=a_{i 4}+e_{i}^{\prime} \cdot x_{1}+f_{i 4} \cdot x_{2}$. Especially, $f_{i}=f_{i 1} \cdot f_{i 2} \cdot f_{i 3} \cdot f_{i 4}$. By minimality of $t$, the conclusion of $\left(\mathrm{S} 2^{\prime}\right)$ holds for $u_{1} u_{4}\left[x_{1}, t_{2} t_{3}\right], u_{1} u_{2} u_{4}\left[x_{1}, t_{2} t_{3}\right]$, and for $u_{1} u_{3} u_{4}\left[x_{1}, t_{2} t_{3}\right]$. This gives us

$$
\begin{aligned}
& f_{11} f_{14} \cdot A_{1}=f_{21} f_{24} \cdot A_{2}, \\
& f_{11} f_{12} f_{14} \cdot A_{1}=f_{21} f_{22} f_{24} \cdot A_{2}, \\
& f_{11} f_{13} f_{14} \cdot A_{1}=f_{21} f_{23} f_{24} \cdot A_{2}
\end{aligned}
$$

for $A_{i}:=n_{i}+\left(d_{i}-1\right) \cdot b_{i}$. First assume either both sides in (2) or in (3) equal 0 . Then both sides in the conclusion of (S2') for $t$ equal 0 as well, in contradiction to our assumption. Therefore, assume all the numbers $f_{i v}$ and $A_{i}$ in (2) and (3) are different from 0 . Powers of a prime can be viewed as members of the free monoid $1 *$. It follows that we can apply Schützenberger's lemma (see Proposition 1.4(ii)S) to (1), (2), and (3) which gives us

$$
f_{1} \cdot A_{1}=f_{11} f_{12} f_{13} f_{14} \cdot A_{1}=f_{21} f_{22} f_{23} f_{24} \cdot A_{2}=f_{2} \cdot A_{2},
$$

in contradiction to our assumption.

Case 1.3: $t_{1}$ contains a proper subtree from $T_{\Sigma}\left(x_{2}\right)$ in which the path to the leaf $x_{2}$ has length $>2 n^{3}$. Then $t_{1}=u_{1}\left[x_{1}, u_{2} u_{3} u_{4} x_{2}\right]$ for proper $u_{1} \in T_{\Sigma}\left(X_{2}\right)$ and $u_{2}$, $u_{3} \in T_{\Sigma}\left(x_{1}\right)$ with $u_{2}, u_{3} \neq x_{1}$ such that

$$
\begin{aligned}
& \left(p, u_{1}, r_{11}, p q_{1}\right),\left(q_{1}, u_{2}, r_{12}, q_{1}\right),\left(q_{1}, u_{3}, r_{13}, q_{1}\right),\left(q_{1}, u_{4}, r_{14}, p_{1}\right), \\
& \left(p, u_{1}, r_{21}, q q_{2}\right),\left(q_{2}, u_{2}, r_{22}, q_{2}\right),\left(q_{2}, u_{3}, r_{23}, q_{2}\right),\left(q_{2}, u_{4}, r_{24}, p_{2}\right), \\
& \left(q, u_{1}, r_{31}, q q_{3}\right),\left(q_{3}, u_{2}, r_{32}, q_{3}\right),\left(q_{3}, u_{3}, r_{33}, q_{3}\right),\left(q_{3}, u_{4}, r_{34}, p_{3}\right) \in \delta
\end{aligned}
$$

for certain states $q_{i}$ and decompositions $s_{i 1}=r_{i 1}\left[x_{1}, r_{i 2} r_{i 3} r_{i 4} x_{2}\right]$.

The argumentation is similar to Case 1.2. Assume

$$
\omega\left(r_{i 1}\right)=a_{i 1}+e_{i} \cdot x_{1}+f_{i 1} \cdot x_{2},
$$

and $\omega\left(r_{i v}\right)=a_{i v}+f_{i v} \cdot x_{1}$ for $v=2,3,4$. Then, especially, $f_{i}=f_{i 1} \cdot f_{i 2} \cdot f_{i 3} \cdot f_{i 4}$. By minimality of $t$, the conclusion of (S2') holds for $u_{1}\left[x_{1}, u_{4} t_{2} t_{3}\right], u_{1}\left[x_{1}, u_{2} u_{4} t_{2} t_{3}\right]$, and for $u_{1}\left[x_{1}, u_{3} u_{4} t_{2} t_{3}\right]$. This again gives us (1), (2), and (3), and then the same argument as in Case 1.2 shows that $f_{1} \cdot A_{1}=f_{2} \cdot A_{2}$. 
Case 1.4: $t_{1}$ contains a proper subtree from $T_{\Sigma}\left(x_{1}\right)$ in which the path to the leaf $x_{1}$ has length $>n^{3}$. Then $t_{1}=u_{1}\left[u_{2} u_{3}, x_{2}\right]$ for proper $u_{1}, u_{2}, u_{3} \in T_{\Sigma}\left(x_{1}\right)$ with $u_{2} \neq$ $x_{1}$ such that

$$
\begin{aligned}
& \left(p, u_{1}, r_{11}, q_{1} p_{1}\right),\left(q_{1}, u_{2}, r_{12}, q_{1}\right),\left(q_{1}, u_{3}, r_{13}, p\right), \\
& \left(p, u_{1}, r_{21}, q_{2} p_{2}\right),\left(q_{2}, u_{2}, r_{22}, q_{2}\right),\left(q_{2}, u_{3}, r_{23}, q\right), \\
& \left(q, u_{1}, r_{31}, q_{3} p_{3}\right),\left(q_{3}, u_{2}, r_{32}, q_{3}\right),\left(q_{3}, u_{3}, r_{33}, q\right) \in \delta
\end{aligned}
$$

for certain states $q_{i}$ and decompositions $s_{i 1}=r_{i 1}\left[r_{i 2} r_{i 3}, x_{2}\right]$.

By minimality of $t, M$ has Property (S2') for $t^{\prime}=u_{1}\left[u_{3}, t_{2} t_{3}\right]$. By definition, $\omega\left(r_{i 1}\left[r_{i 3}, x_{2}\right]\right)=a_{i}^{\prime}+e_{i}^{\prime} \cdot x_{1}+f_{i}^{\prime} \cdot x_{2}$ for certain $a_{i}^{\prime}$, $e_{i}^{\prime}$, and $f_{i}^{\prime}$ where $f_{i}^{\prime}=f_{i}$. Whence, $\left(\mathrm{S} 2^{\prime}\right)$ for $t^{\prime}$ implies (S2') for $t$, in contradiction to our assumption.

Case 2: $t_{2}$ is too "large."

Case 2.1: The path in $t_{2}$ to the leaf $x_{1}$ has length $>n^{3}$. Then $t_{2}=u_{1} u_{2} u_{3}$ for proper $u_{1}, u_{2}, u_{3} \in T_{\Sigma}\left(x_{1}\right)$ with $u_{2} \neq x_{1} \neq u_{1} u_{3}$ such that

$$
\left(p_{i}, u_{1}, r_{i 1}, q_{i}\right),\left(q_{i}, u_{2}, r_{i 2}, q_{i}\right),\left(q_{i}, u_{3}, r_{i 3}, p_{i}\right) \in \delta, \quad \text { for } \quad i=1,2,3 \text {, }
$$

for certain states $q_{i}$ and decompositions $s_{i 2}=r_{i 1} r_{i 2} r_{i 3}$. Assume $\omega\left(r_{i v}\right)=$ $n_{i v}+d_{i v} \cdot x_{1}$ for $i=1,2$ and $v=1,2,3$.

By minimality of $t, M$ has Property (S2') for $t^{\prime}=t_{1}\left[x_{1}, u_{1} u_{3} t_{3}\right]$. Whence

$$
\begin{aligned}
& f_{1} n_{11}+f_{1} d_{11} n_{13}+f_{1}\left(d_{11} d_{13}-1\right) b_{1} \\
& \quad=f_{2} n_{21}+f_{2} d_{21} n_{23}+f_{2}\left(d_{21} d_{23}-1\right) b_{2} .
\end{aligned}
$$

Moreover, by minimality of $t_{2}$, we find that $M$ allows for conclusion (S2') for $t$ factored $t=t_{1}^{\prime}\left[x_{1}, t_{2}^{\prime} t_{3}^{\prime}\right]$ where $t_{1}^{\prime}:=t_{1}\left[x_{1}, u_{1} x_{2}\right], t_{2}^{\prime}:=u_{2}$, and $t_{3}^{\prime}:=u_{3} t_{3}$. Whence

$$
\begin{aligned}
& f_{1} d_{11} n_{12}+f_{1} d_{11}\left(d_{12}-1\right)\left[n_{13}+d_{13} b_{1}\right] \\
& \quad=f_{2} d_{21} n_{22}+f_{2} d_{21}\left(d_{22}-1\right)\left[n_{23}+d_{23} b_{2}\right] .
\end{aligned}
$$

Therefore,

$$
\begin{aligned}
f_{2} \cdot[ & \left.n_{2}+\left(d_{2}-1\right) b_{2}\right]-f_{1} \cdot\left[n_{1}+\left(d_{1}-1\right) b_{1}\right] \\
= & f_{2}\left[n_{21}+d_{21} n_{22}+d_{21} d_{22} n_{23}\right]+f_{2}\left(d_{21} d_{22} d_{23}-1\right) b_{2} \\
& \quad-f_{1}\left[n_{11}+d_{11} n_{12}+d_{11} d_{12} n_{13}\right]-f_{1}\left(d_{11} d_{12} d_{13}-1\right) b_{1} \\
& \stackrel{(2)}{=} f_{2} n_{21}+f_{2} d_{21} n_{23}+f_{2}\left(d_{21} d_{23}-1\right) b_{2} \\
& \quad-f_{1} n_{11}-f_{1} d_{11} n_{13}-f_{1}\left(d_{11} d_{13}-1\right) b_{1} \\
& \stackrel{(1)}{=} 0
\end{aligned}
$$

which implies that $M$ also allows for conclusion (S2') for $t$ factored $t=t_{1}\left[x_{1}, t_{2} t_{3}\right]$, in contradiction to our assumption. 
Case 2.2: $t_{2}$ contains a subtree from $T_{\Sigma}$ of depth $>n^{3}$. Then $t_{2}=u_{1}\left[x_{1}, u_{2} u_{3}\right]$ for proper $u_{1} \in T_{\Sigma}\left(X_{2}\right)$, proper $u_{2} \in T_{\Sigma}\left(x_{1}\right)$, and $u_{3} \in T_{\Sigma}$ with $u_{2} \neq x_{1}$ such that

$$
\left(p_{i}, u_{1}, r_{i 1}, p_{i} q_{i}\right),\left(q_{i}, u_{2}, r_{i 2}, q_{i}\right),\left(q_{i}, u_{3}, r_{i 3}, \varepsilon\right) \in \delta, \quad \text { for } \quad i=1,2,3,
$$

for certain states $q_{i}$ and decompositions $s_{i 2}=r_{i 1}\left[x_{1}, r_{i 2} r_{i 3}\right]$. Assume

$$
\begin{aligned}
& \omega\left(r_{i 1}\right)=n_{i 1}+d_{i} \cdot x_{1}+f_{i}^{\prime} \cdot x_{2}, \\
& \omega\left(r_{i 2}\right)=n_{i 2}+d_{i}^{\prime} \cdot x_{1}, \\
& \omega\left(r_{i 3}\right)=n_{i 3} \quad \text { for } \quad i=1,2 .
\end{aligned}
$$

By minimality of $t, M$ has Property $\left(\mathbf{S} 2^{\prime}\right)$ for $t^{\prime}:=t_{1}\left[x_{1}, u_{1}\left[t_{3}, u_{3}\right]\right]$. Whence

$$
f_{1}\left[n_{11}+f_{1}^{\prime} n_{13}\right]+f_{1}\left(d_{1}-1\right) b_{1}=f_{2}\left[n_{21}+f_{2}^{\prime} n_{23}\right]+f_{2}\left(d_{2}-1\right) b_{2} .
$$

By minimality of $t_{2}$, we may assume that $M$ allows for the conclusion (S2') for $t$ with respect to the factorization $t=t_{1}^{\prime}\left[x_{1}, t_{2}^{\prime}, t_{3}^{\prime}\right]$ where $t_{1}^{\prime}:=t_{1}\left[x_{1}, u_{1}\left[t_{3}, x_{2}\right]\right]$, $t_{2}^{\prime}:=u_{2}$, and $t_{3}^{\prime}:=u_{3}$. This gives us

$$
f_{1} f_{1}^{\prime} n_{12}+f_{1} f_{1}^{\prime}\left(d_{1}^{\prime}-1\right) n_{13}=f_{2} f_{2}^{\prime} n_{22}+f_{2} f_{2}^{\prime}\left(d_{2}^{\prime}-1\right) n_{23} .
$$

Therefore,

$$
\begin{aligned}
f_{2} \cdot & {\left[n_{2}+\left(d_{2}-1\right) b_{2}\right]-f_{1} \cdot\left[n_{1}+\left(d_{1}-1\right) b_{1}\right] } \\
= & f_{2}\left[n_{21}+f_{2}^{\prime} n_{22}+f_{2}^{\prime} d_{2}^{\prime} n_{23}\right]+f_{2}\left(d_{2}-1\right) b_{2} \\
& \quad-f_{1}\left[n_{11}+f_{1}^{\prime} n_{12}+f_{1}^{\prime} d_{1}^{\prime} n_{13}\right]-f_{1}\left(d_{1}-1\right) b_{1} \\
& \stackrel{(2)}{=} f_{2}\left[n_{21}+f_{2}^{\prime} n_{23}\right]+f_{2}\left(d_{2}-1\right) b_{2}-f_{1}\left[n_{11}+f_{1}^{\prime} n_{13}\right]-f_{1}\left(d_{1}-1\right) b_{1} \\
& \stackrel{(1)}{=} 0 .
\end{aligned}
$$

Whence, $M$ allows for the conclusion (S2') also for $t$ with respect to factorization $t=t_{1}\left[x_{1}, t_{2} t_{3}\right]$, in contradiction to our assumption.

Case 3: $t_{3}$ has depth $>n^{3}$. Then $t_{3}=u_{1} u_{2} u_{3}$ for proper $u_{1}, u_{2} \in T_{\Sigma}\left(x_{1}\right)$ and $u_{3} \in T_{\Sigma}$ with $u_{2} \neq x_{1}$ such that

$$
\left(p_{i}, u_{1}, r_{i 1}, q_{i}\right),\left(q_{i}, u_{2}, r_{i 2}, q_{i}\right),\left(q_{i}, u_{3}, r_{i 3}, \varepsilon\right) \in \delta
$$

for $i=1,2,3$, states $q_{1}, q_{2}, q_{3} \in Q$, and decompositions $s_{i 3}=r_{i 1} r_{i 2} r_{i 3}$.

We may assume that $t_{2} \neq x_{1}$ because otherwise the conclusion of (S2') trivially holds. Therefore, by minimality of $t, M$ has Property (S2') both for

$$
t^{\prime}=t_{1}\left[x_{1}, t_{2} u_{1} u_{3}\right]
$$

factorized in the obvious way and for $t^{\prime \prime}=t_{1}\left[x_{1}, u_{1} u_{2} u_{3}\right]$ with factors $t_{1}\left[x_{1}, u_{1} x_{2}\right]$, $u_{2}$, and $u_{3}$. Assuming $\omega\left(r_{i v}\right)=b_{i v}+d_{i v} \cdot x_{1}$ for $v=1,2,3$ we deduce

$$
\begin{aligned}
& f_{1} \cdot\left[n_{1}+\left(d_{1}-1\right) \cdot\left(b_{11}+d_{11} b_{13}\right)\right]=f_{2} \cdot\left[n_{2}+\left(d_{2}-1\right) \cdot\left(b_{21}+d_{21} b_{23}\right)\right], \\
& f_{1} \cdot d_{11} \cdot\left[b_{12}+\left(d_{12}-1\right) \cdot b_{13}\right]=f_{2} \cdot d_{21}\left[b_{22}+\left(d_{22}-1\right) \cdot b_{23}\right] .
\end{aligned}
$$


Using (1) and (2) we find

$$
\begin{aligned}
f_{1} \cdot & {\left[n_{1}+\left(d_{1}-1\right) \cdot b_{1}\right] } \\
& =f_{1} \cdot\left[n_{1}+\left(d_{1}-1\right) \cdot\left(b_{11}+d_{11} b_{12}+d_{11} d_{12} b_{13}\right)\right] \\
& \left.=f_{1} \cdot\left[n_{1}+\left(d_{1}-1\right) \cdot\left(b_{11}+d_{11} b_{13}\right)\right]+f_{1} d_{11} \cdot\left[b_{12}+\left(d_{12}-1\right) b_{13}\right)\right] \\
& \left.=f_{2} \cdot\left[n_{2}+\left(d_{2}-1\right) \cdot\left(b_{21}+d_{21} b_{23}\right)\right]+f_{2} d_{21} \cdot\left[b_{22}+\left(d_{22}-1\right) b_{23}\right)\right] \\
& =f_{2} \cdot\left[n_{2}+\left(d_{2}-1\right) \cdot b_{2}\right],
\end{aligned}
$$

in contradiction to our assumption.

(iii). Before we prove Proposition 4.2(iii), we consider two auxiliary facts which show that we can eliminate subcomputations in a controlled way without changing equality in the outputs produced.

Fact 4. Assume $M$ has Property (S1'). Given states $p, q, q_{1}, q_{2}, q_{3}, q_{4} \in Q$, decompositions $t=u_{1} u_{2} u_{3}$ for proper trees $u_{v}$, and $s_{i}=r_{i 1} r_{i 2} r_{i 3}, i=1,2,3,4$, such that

$$
\begin{aligned}
& \left(p, u_{1}, r_{11}, q_{1}\right),\left(q_{1}, u_{2}, r_{12}, q_{1}\right),\left(q_{1}, u_{3}, r_{13}, p\right), \\
& \left(p, u_{1}, r_{21}, q_{2}\right),\left(q_{2}, u_{2}, r_{22}, q_{2}\right),\left(q_{2}, u_{3}, r_{23}, q\right), \\
& \left(p, u_{1}, r_{31}, q_{3}\right),\left(q_{3}, u_{2}, r_{32}, q_{3}\right),\left(q_{3}, u_{3}, r_{33}, q\right), \\
& \left(q, u_{1}, r_{41}, q_{4}\right),\left(q_{4}, u_{2}, r_{42}, q_{4}\right),\left(q_{4}, u_{3}, r_{43}, q\right) \in \delta,
\end{aligned}
$$

then $s_{1} \notin\left\{\perp, x_{1}\right\}$ and $\omega\left(s_{2}\right)=\omega\left(s_{3}\right)$ implies $\omega\left(r_{21} r_{23}\right)=\omega\left(r_{31} r_{33}\right)$.

\section{Proof. Let}

$$
\begin{aligned}
& \omega\left(r_{i 1}\right)=a_{i}+e_{i} \cdot x_{1}, \\
& \omega\left(r_{i 2}\right)=n_{i}+d_{i} \cdot x_{1}, \\
& \omega\left(r_{i 3}\right)=b_{i}+f_{i} \cdot x_{1}
\end{aligned}
$$

and

$$
\omega\left(r_{i 1} r_{i 3}\right)=A_{i}+B_{i} \cdot x_{1} \quad \text { for } \quad i=1,2,3,4 .
$$

Thus $\omega\left(s_{2}\right)=\omega\left(s_{3}\right)$ translates to

$$
e_{2} d_{2} f_{2}=e_{3} d_{3} f_{3}
$$

and

$$
a_{2}+e_{2} n_{2}+e_{2} d_{2} b_{2}=a_{3}+e_{3} n_{3}+e_{3} d_{3} b_{3} .
$$

$M$ has Property (S0) since $M$ has Property (S1'). Therefore, $\omega\left(s_{1}\right) \notin\left\{0, x_{1}\right\}$ also 
implies that $\omega\left(s_{4}\right) \notin\left\{0, x_{1}\right\}$. Hence, $q \notin \operatorname{Const}(M)$, and Property $\left(\mathrm{S}^{\prime}\right)$ implies that $d_{1}=d_{2}=d_{3}=: d>0$. Therefore, $B_{2}=e_{2} f_{2}=e_{3} f_{3}=B_{3}$. Moreover,

$$
\begin{aligned}
& (d-1)\left[a_{2}-a_{1}\right]=e_{2} n_{2}-e_{1} n_{1}, \\
& (d-1)\left[a_{3}-a_{1}\right]=e_{3} n_{3}-e_{1} n_{1},
\end{aligned}
$$

and hence

$$
(d-1)\left[a_{3}-a_{2}\right]=e_{3} n_{3}-e_{2} n_{2} .
$$

Hence, $a_{2}+e_{2} n_{2}+e_{2} d_{2} b_{2}=a_{3}+e_{3} n_{3}+e_{3} d_{3} b_{3}$ implies

$$
A_{2}=a_{2}+e_{2} b_{2}=a_{3}+e_{3} b_{3}=A_{3} \text {. }
$$

Fact 5. Assume $M$ has Property (S2'). Given states $p, q, q_{1}, q_{2}, q_{3}, q_{4} \in Q$, decompositions $t=u_{1}\left[x_{1}, u_{2} u_{3}\right]$ for proper trees $u_{1}, u_{2}$, and $s_{i}=r_{i 1}\left[x_{1}, r_{i 2} r_{i 3}\right]$, $i=1,2,3,4$, such that

$$
\begin{aligned}
& \left(p, u_{1}, r_{11}, p q_{1}\right),\left(q_{1}, u_{2}, r_{12}, q_{1}\right),\left(q_{1}, u_{3}, r_{13}, \varepsilon\right), \\
& \left(p, u_{1}, r_{21}, q q_{2}\right),\left(q_{2}, u_{2}, r_{22}, q_{2}\right),\left(q_{2}, u_{3}, r_{23}, \varepsilon\right), \\
& \left(p, u_{1}, r_{31}, q q_{3}\right),\left(q_{3}, u_{2}, r_{32}, q_{3}\right),\left(q_{3}, u_{3}, r_{33}, \varepsilon\right), \\
& \left(q, u_{1}, r_{41}, q q_{4}\right),\left(q_{4}, u_{2}, r_{42}, q_{4}\right),\left(q_{4}, u_{3}, r_{43}, \varepsilon\right) \in \delta,
\end{aligned}
$$

then $\omega\left(s_{2}\right)=\omega\left(s_{2}\right)$ implies $\omega\left(r_{21}\left[x, r_{23}\right]\right)=\omega\left(r_{31}\left[x_{1}, r_{33}\right]\right)$.

\section{Proof. Define}

$$
\begin{aligned}
& \omega\left(r_{i 1}\right)=a_{i}+e_{i} x_{i}+f_{i} x_{2}, \\
& \omega\left(r_{i 2}\right)=n_{i}+d_{i} x_{1}, \\
& \omega\left(r_{i 3}\right)=b_{i}, \\
& \omega\left(r_{i 1}\left[x_{1}, r_{i 3}\right]\right)=A_{i}+B_{i} x_{1} \quad \text { for } \quad i=1,2,3,4 .
\end{aligned}
$$

Certainly, $B_{2}=e_{2}=e_{3}=B_{3}$.

$$
\text { Moreover, Property (S2') implies }
$$

$$
f_{1} n_{1}+\left(d_{1}-1\right) f_{1} b_{1}=f_{2} n_{2}+\left(d_{2}-1\right) f_{2} b_{2}=f_{3} n_{3}+\left(d_{3}-1\right) f_{3} b_{3} .
$$

Hence, $a_{2}+f_{2} n_{2}+f_{2} d_{2} b_{2}=a_{3}+f_{3} n_{3}+f_{3} d_{3} b_{3}$ implies

$$
A_{2}=a_{2}+f_{2} b_{2}=a_{3}+f_{3} b_{3}=A_{3} \text {. }
$$

This finishes the proof of Fact 5 .

Now we are prepared to prove Proposition 4.2(iii). Assume $M$ has Properties $\left(\mathrm{S} 1^{\prime}\right)$ and $\left(\mathrm{S} 2^{\prime}\right)$ and Property $\left(\mathrm{FO}^{\prime}\right)$ for trees up to depth $6 n^{4}$. Consider $p, q \in Q$, and some proper tree $t \in T_{\Sigma}\left(x_{1}\right)$ of minimal $\Sigma$-size such that $s_{i} \in T_{\Sigma}\left(x_{1}\right)$ exist with $\left(p, t, s_{1}, p\right),\left(p, t, s_{2}, q\right),\left(p, t, s_{3}, q\right),\left(q, t, s_{4}, q\right) \in \delta$ where $s_{1} \notin\left\{\perp, x_{1}\right\}, \omega\left(s_{2}\right)=\omega\left(s_{3}\right)$, 
but $s_{2} \neq s_{3}$. By assumption, depth $(t)>6 n^{4}$. Therefore, one of the following two cases must occur.

Case 1: The path in to the leaf $x_{1}$ has length $>3 n^{4}$. Then $t=u_{1} u_{2} u_{3} u_{4} u_{5}$ for proper trees $u_{v} \in T_{\Sigma}\left(x_{1}\right)$ with $u_{2}, u_{3}, u_{4} \neq x_{1}$ such that

$$
\begin{aligned}
& \left(p, u_{1}, r_{11}, q_{1}\right),\left(q_{1}, u_{5}, r_{15}, p\right), \\
& \left(p, u_{1}, r_{21}, q_{2}\right),\left(q_{2}, u_{5}, r_{25}, q\right), \\
& \left(p, u_{1}, r_{31}, q_{3}\right),\left(q_{3}, u_{5}, r_{35}, q\right), \\
& \left(q, u_{1}, r_{41}, q_{4}\right),\left(q_{4}, u_{5}, r_{45}, q\right), \\
& \left(q_{i}, u_{v}, r_{i v}, q_{i}\right) \in \delta \quad \text { for } \quad i=1,2,3,4 \text { and } v=2,3,4,
\end{aligned}
$$

for certain states $q_{i}$ and decompositions $s_{i}=r_{i 1} r_{i 2} r_{i 3} r_{i 4} r_{i 5}$. First assume $r_{12}=x_{1}$. Then $\omega\left(r_{22}\right)=\omega\left(r_{32}\right)=x_{1}$ since $M$ has Property ( $\left.\mathrm{S}^{\prime}\right)$. It follows that indeed $r_{22}=r_{32}=x_{1}$. Hence, $s_{i}=r_{i 1} r_{i 3} r_{i 4} r_{i 5}$ for $i=1,2$, 3. It also follows that Property (F0') does not hold for $t^{\prime}:=u_{1} u_{3} u_{4} u_{5}$ where $\left|t^{\prime}\right|_{\Sigma}<|t|_{\Sigma}$, in contradiction to the minimality of $t$.

Therefore, we may assume $r_{12} \neq x_{1}$. Then also $\left(r_{11} r_{12}\right) r_{15},\left(r_{11} r_{12}\right) r_{13} r_{15}$, and $\left(r_{11} r_{12}\right) r_{14} r_{15} \notin\left\{\perp, x_{1}\right\}$. Therefore, by Fact 4 ,

$$
\begin{aligned}
& \omega\left(\left(r_{21}, r_{22}\right) r_{25}\right)=\omega\left(\left(r_{31} r_{32}\right) r_{35}\right), \\
& \omega\left(\left(r_{21}, r_{22}\right) r_{23} r_{25}\right)=\omega\left(\left(r_{31} r_{22}\right) r_{33} r_{35}\right),
\end{aligned}
$$

and

$$
\omega\left(\left(r_{21} r_{22}\right) r_{24} r_{25}\right)=\omega\left(\left(r_{31} r_{22}\right) r_{34} r_{35}\right) .
$$

Since $t$ was chosen minimal, Property (F0) holds for $\left(u_{1} u_{2}\right) u_{5}$, for $\left(u_{1} u_{2}\right) u_{3} u_{5}$, and for $\left(u_{1} u_{2}\right) u_{4} u_{5}$. We conclude that

$$
\begin{aligned}
& \left(r_{21} r_{22}\right) r_{25}=\left(r_{31} r_{32}\right) r_{35}, \\
& \left(r_{21} r_{22}\right) r_{23} r_{25}=\left(r_{31} r_{32}\right) r_{33} r_{35},
\end{aligned}
$$

and

$$
\left(r_{21} r_{22}\right) r_{24} r_{25}=\left(r_{31} r_{32}\right) r_{34} r_{35} \text {. }
$$

Thus, the assumptions of Proposition 1.4(ii) are satisfied which implies that

$$
s_{2}=r_{21} r_{22} r_{23} r_{24} r_{25}=r_{31} r_{32} r_{33} r_{34} r_{35}=s_{3} \text {, }
$$

in contradiction to our assumption. 
Case 2: $t$ contains a subtree from $T_{\Sigma}$ of depth $>3 n^{4}$. Then $t=u_{1}\left[x_{1}, u_{2} u_{3} u_{4} u_{5}\right]$ for proper trees $u_{v} \neq x_{1}, v=2,3,4$, such that

$$
\begin{aligned}
& \left(p, u_{1}, r_{11}, p q_{1}\right),\left(q_{1}, u_{5}, r_{15}, \varepsilon\right), \\
& \left(p, u_{1}, r_{21}, q q_{2}\right),\left(q_{2}, u_{5}, r_{25}, \varepsilon\right), \\
& \left(p, u_{1}, r_{31}, q q_{3}\right),\left(q_{3}, u_{5}, r_{35}, \varepsilon\right), \\
& \left(q, u_{1}, r_{41}, q q_{4}\right),\left(q_{4}, u_{5}, r_{45}, \varepsilon\right), \\
& \left(q_{i}, u_{v}, r_{i v}, q_{i}\right) \in \delta \quad \text { for } \quad i=1,2,3,4 \text { and } v=2,3,4,
\end{aligned}
$$

for certain states $q_{i}$ and decompositions $s_{i}=r_{i 1}\left[x_{1}, r_{i 2} r_{i 3} r_{i 4} r_{i 5}\right]$. By Fact 5 , $\omega\left(s_{2}\right)=\omega\left(s_{3}\right)$ implies that

$$
\omega\left(r_{21}\left[x_{1}, r_{25}\right]\right)=\omega\left(r_{31}\left[x_{1}, r_{35}\right]\right) .
$$

Since $t$ was chosen of minimal $\Sigma$-size, $M$ has Property $\left(F 0^{\prime}\right)$ for $u_{1}\left[x_{1}, u_{5}\right]$. Hence,

$$
r_{21}\left[x_{1}, r_{25}\right]=r_{31}\left[x_{1}, r_{35}\right] \text {. }
$$

Analogously, we deduce that

$$
\begin{aligned}
& r_{21}\left[x_{1}, r_{22} r_{25}\right]=r_{31}\left[x_{1}, r_{32} r_{35}\right], \\
& r_{21}\left[x_{1}, r_{24} r_{25}\right]=r_{31}\left[x_{1}, r_{34} r_{35}\right], \\
& r_{21}\left[x_{1}, r_{23} r_{24} r_{25}\right]=r_{31}\left[x_{1}, r_{33} r_{34} r_{35}\right], \\
& r_{21}\left[x_{1}, r_{22} r_{23} r_{25}\right]=r_{31}\left[x_{1}, r_{32} r_{33} r_{35}\right],
\end{aligned}
$$

and

$$
r_{21}\left[x_{1}, r_{22} r_{24} r_{25}\right]=r_{31}\left[x_{1}, r_{32} r_{34} r_{35}\right] \text {. }
$$

Thus, we can apply Proposition 1.4(i) to derive

$$
s_{2}=r_{21}\left[x_{1}, r_{22} r_{23} r_{24} r_{25}\right]=r_{31}\left[x_{1}, r_{32} r_{33} r_{34} r_{35}\right]=s_{3},
$$

in contradiction to our assumption. This finishes the proof.

\section{Upper Bounds for Finite (Size-) Valuedness}

In this section we show that Properties (F1) and (F2) (Properties (S1) and (S2)) are not only necessary but also sufficient conditions for an FST $M$ to be finite-valued (finite size-valued). In showing this we prove:

- $\operatorname{sval}(M)<\infty$ implies $\operatorname{sval}(M)<2^{2^{c|M| \cdot \log (L+1)}}$ for some constant $c>0$ independent of $M$ (Theorem 5.4).

- $\operatorname{val}(M)<\infty$ implies $\operatorname{val}(M)<2^{2^{P(M M)}}$ for some polynomial $P$ independent of $M$ (Theorem 5.5). 
The proofs are inductions on the number of strong components of $M$. The bases of these inductions are given by Proposition 5.1. For the inductive step we classify the accepting computations of $M$ according to their behavior between nodes with identical sets of accessible and derivable states (Proposition 5.2).

Assume $p, q \in Q . q$ is called reachable from $p$ (denoted $p \rightarrow_{M} q$ ) iff there is a $(p, q)$-computation of $M$ for some proper tree, $q$ and $p$ are connected (denoted $q \leftrightarrow_{M} p$ ) iff $p \rightarrow_{M} q$ and $q \rightarrow_{M} p$. Clearly, $\leftrightarrow_{M}$ is an equivalence relation on $Q$. The equivalence classes $Q_{1}, \ldots, Q_{k}$ of $Q$ are also called strong components of $M$. Without loss of generality, they are numbered in such a way that, for all $p \in Q_{i}, q \in Q_{j}$, $p \rightarrow_{M} q$ implies $i \leq j$. Proposition 5.1 investigates the case of just one strong component.

Proposition 5.1. Assume $p, q \in Q$ are connected, and $\left(p, t, s_{1}, q\right),\left(p, t, s_{2}, q\right) \in \delta$ for some proper $t \in T_{\Sigma}\left(x_{1}\right)$ :

(i) If $M$ has Property (S0), then $\omega\left(s_{1}\right)=\omega\left(s_{2}\right)$.

(ii) If $M$ has Property (F0), then $s_{1}=s_{2}$.

Proof. We only consider statement (ii). Without loss of generality, let $t \neq x_{1}$. If $p \in U(M)$, then $q \in U(M)$ as well, and $s_{1}=\perp=s_{2}$ which proves the assertion. If $p \notin U(M)$, then also $q \notin U(M)$ since $p$ and $q$ are connected. Moreover, $(q, u, v, p) \in \delta$ for some proper $u$ and some $v$ in which $x_{1}$ occurs. We consider $t^{\prime}:=t u$. Then $\left(p, t u, s_{1} v, p\right),\left(p, t u, s_{1} v, p\right),\left(p, t u, s_{2} v, p\right) \in \delta$. Thus the conclusion of (F0) gives

$$
s_{1} v s_{1} v=s_{1} v s_{2} v .
$$

From this, assertion (ii) follows by top and bottom cancellation according to Proposition 1.1S.

Proposition 5.2. $A$ constant $c>0$ and a polynomial $P$ independent of $M$ exist such that, for proper $t \in T_{\Sigma}\left(x_{1}\right)$ and $B \subseteq Q$ with,

$\forall p \in B, \quad \exists q \in B, \quad \exists(p, q)$-computation of $M$ for $t$,

$\forall q \in B, \quad \exists p \in B, \quad \exists(p, q)$-computation of $M$ for $t$, the following holds:

(i) If $M$ has Properties (S1) and (S2), then

$$
\#\{\omega(s) \mid(p, t, s, q) \in \delta\}<2^{2^{c \cdot|M| \log (L+1)}} \quad \text { for every } \quad p, q \in B .
$$

(ii) If $M$ has Properties (F1) and (F2), then

$$
\#\{s \mid(p, t, s, q) \in \delta\}<2^{2^{P(M \mid)}} \quad \text { for every } \quad p, q \in B .
$$

The proof in [W4] of the (single exponential) result for NGSMs corresponding to Proposition 5.2 is based on the observation that the difference between lengths of possible output words is appropriately bounded. This is no longer true for trees. 
As a substitute, we prove that the output only depends on the kernel of a suitable triple of computations.

Let $q_{i}, p_{i, 1}, \ldots, p_{i, k} \in Q$ and let $\varphi_{i}$ be a $\left(q_{i}, p_{i, 1} \cdots p_{i, k}\right)$ computation of $M$ for $t \in T_{\Sigma}\left(X_{k}\right), i=1,2,3$. Then the triple $\varphi=\left\langle\varphi_{1}, \varphi_{2}, \varphi_{3}\right\rangle$ is called $\left(z, z_{1} \cdots z_{k}\right)$ computation of $M^{3}$ for $t$ where $z=\left\langle q_{1}, q_{2}, q_{3}\right\rangle$ and $z_{j}=\left\langle p_{1, j}, p_{2, j} p_{3, j}\right\rangle$. Such a computation can also be viewed as a tree in $T_{\delta^{3}}\left(X_{k}\right)$ where $\delta^{3}$ consists of all triples $\left\langle\tau_{1}, \tau_{2}, \tau_{3}\right\rangle$ of transitions $\tau_{i}=\left(q_{i}, a_{i}, s_{i}, q_{i, 1} \cdots q_{i, m}\right) \in \delta$ with $a_{1}=a_{2}=a_{3}$. Such a triple is now called a $\left\langle q_{1}, q_{2}, q_{3}\right\rangle$-transition. According to this definition, computations of $M^{3}$ can be composed and decomposed similar to trees or computations of $M$ itself.

For a computation $\varphi=\left\langle\varphi_{1}, \varphi_{2}, \varphi_{3}\right\rangle$ of $M^{3}$ and $i \in\{1,2,3\}$, the $i$ th output $T_{i}(\varphi)$ is defined as $T\left(\varphi_{i}\right)$. Analogously, $\Omega_{i}(\varphi):=\Omega\left(\varphi_{i}\right)$. Assume $\varphi$ is a computation of $M^{3}$ for some proper tree $t \in T_{\Sigma}\left(x_{1}\right)$. Intuitively, the kernel $\mathbf{c} \varphi$ of $\varphi$ is obtained from $\varphi$ by eliminating proper $(z, z)$-computations. This is done in two steps.

First assume $\varphi$ is a $z$-computation of $M^{3}, v \in T_{\Sigma}$, and we have the recursive decomposition $\varphi_{r}=\psi_{r} \tau_{r}\left(\varphi_{r \cdot 1}, \ldots, \varphi_{r \cdot m}\right), r \in O(v)$, where $m_{r}$ is the rank of $v(r)$ and $\tau_{r} \in \delta^{3}$, and $\varphi_{\varepsilon}=\varphi$ such that, for every $r \in O(v)$, some $p \in Q^{3}$ exists with:

(1) $\psi_{r}$ is a proper $(p, p)$-computation.

(2) For no $j \in\left\{1, \ldots, m_{r}\right\}, \varphi_{r j}$ contains a $p$-transition.

(3) If $\psi_{r}(o)=x_{1}$ and $\psi_{r}\left(o^{\prime}\right)$ is a $p$-transition, then $o$ is lexicographically smaller than $o^{\prime}$.

Then $\mathbf{c} \varphi$ is obtained from $\varphi$ by removing the subcomputations $\psi_{r}, r \in O(v)$. Hence, $\mathbf{c} \varphi$ is defined by $\mathbf{c} \varphi:=\mathbf{c} \varphi_{\varepsilon}$ where, for $r \in O(v), \mathbf{c} \varphi_{r}:=\tau_{r}\left(\mathbf{c} \varphi_{r 1}, \ldots, \mathbf{c} \varphi_{r r_{r}}\right)$.

Now assume we are given a proper $\left(z, z^{\prime}\right)$-computation $\varphi$ of $M^{3}$. Consider the recursive decomposition $\varphi_{\kappa}=\psi_{\kappa} \tau_{\kappa}\left(\varphi_{k, 1}, \ldots, \varphi_{\kappa, m_{k}}\right), \kappa=1, \ldots, k$, where $\varphi_{1}=\varphi$, $\varphi_{k+1}=x_{1}$, and, for every $\kappa \in\{1, \ldots, k\}$, some $p \in Q^{3}$ and $j \in \mathbb{N}$ exist such that:

(1) $\varphi_{\kappa+1}=\varphi_{\kappa, j}$.

(2) $\psi_{\kappa}$ is a proper $(p, p)$-computation.

(3) No proper subcomputation of $\varphi_{\kappa+1}$ is a $\left(p, z^{\prime}\right)$-computation.

Then the kernel of $\varphi, \mathbf{c} \varphi$, is obtained from $\varphi$ by removing the subcomputations $\psi_{\kappa}$ and applying $\mathbf{c}(-)$ to the subcomputations $\varphi_{\kappa, j^{\prime}}$ which do not contain $x_{1}$. Hence, $\mathbf{c} \varphi$ is defined by $\mathbf{c} \varphi:=\mathbf{c} \varphi_{1}$ where $\mathbf{c} x_{1}:=x_{1}$, and, for $\kappa=1, \ldots, k, \mathbf{c} \varphi_{\kappa}:=$ $\tau_{k}\left(\mathbf{c} \varphi_{\kappa, 1}, \ldots, \mathbf{c} \varphi_{k, m_{k}}\right)$.

Proposition 5.3. Assume $M$ has Properties (S1) and (S2). Assume $t \in T_{\Sigma}\left(x_{1}\right)$ is proper, $z=\langle p, p, q\rangle, z^{\prime}=\langle p, q, q\rangle$ for some $p, q \in Q$, and $\varphi$ is a $\left(z, z^{\prime}\right)$-computation of $M^{3}$ for $t$. Assume $t=t_{1} t_{2} t_{3}$ is a decomposition of $t$ into proper trees and $\varphi=\varphi_{1} \varphi_{2} \varphi_{3}$ is the corresponding decomposition of the computation $\varphi$ where $\varphi_{2}$ is $a\left(z_{1}, z_{2}\right)$ computation of $M^{3}$. Let $\psi$ denote the kernel of $\varphi_{2}$, and $\tilde{\varphi}=\varphi_{1} \psi \varphi_{3}$.

(i) If $\Omega_{1}\left(\varphi_{2}\right) \in\left\{0, x_{1}\right\}$, then $T_{2}\left(\varphi_{2}\right)=T_{2}(\psi)$.

(ii) If $q \notin \operatorname{Const}(M)$, then $\Omega_{1}(\varphi)^{-1} \Omega_{2}(\varphi)=\Omega_{1}(\tilde{\varphi})^{-1} \Omega_{2}(\tilde{\varphi})$.

(iii) Let $\varphi_{2}^{\prime}$ be another $\left(z_{1}, z_{2}\right)$-computation of $M^{3}$ for $t_{2}$, and let $\psi^{\prime}$ be the kernel of $\varphi_{2}^{\prime}$. Then $\Omega_{i}(\psi)=\Omega_{i}\left(\psi^{\prime}\right)$ for $i=1,2$ implies $\Omega_{2}\left(\varphi_{2}\right)=\Omega_{2}\left(\varphi_{2}^{\prime}\right)$. 
Proof. First we prove that statement (iii) follows from (i) and (ii). If $\Omega_{1}\left(\varphi_{2}\right) \in$ $\left\{0, x_{1}\right\}$, then $\Omega_{1}\left(\varphi_{2}^{\prime}\right) \in\left\{0, x_{1}\right\}$ as well. Then the assertion directly follows from (i). If $\Omega_{1}\left(\varphi_{2}\right) \notin\left\{0, x_{1}\right\}$, then $q$ cannot be in $\operatorname{Const}(M)$. We conclude from (ii) that

$$
\begin{aligned}
\Omega_{2}\left(\varphi_{2}\right) & =\Omega_{2}\left(\varphi_{1}\right)^{-1} \Omega_{1}(\varphi) \Omega_{1}(\tilde{\varphi})^{-1} \Omega_{2}(\tilde{\varphi}) \Omega_{2}\left(\varphi_{3}\right)^{-1} \\
& =\Omega_{2}\left(\varphi_{1}\right)^{-1} \Omega_{1}(\varphi) \Omega_{1}\left(\varphi_{3}\right)^{-1} \Omega_{1}(\psi)^{-1} \Omega_{1}\left(\varphi_{1}\right)^{-1} \Omega_{2}\left(\varphi_{1}\right) \Omega_{2}(\psi) .
\end{aligned}
$$

Let $\varphi^{\prime}=\varphi_{1} \varphi_{2}^{\prime} \varphi_{3}$. Analogously, we find

$$
\Omega_{2}\left(\varphi_{2}^{\prime}\right)=\Omega_{2}\left(\varphi_{1}\right)^{-1} \Omega_{1}\left(\varphi^{\prime}\right) \Omega_{1}\left(\varphi_{3}\right)^{-1} \Omega_{1}\left(\psi^{\prime}\right)^{-1} \Omega_{1}\left(\varphi_{1}\right)^{-1} \Omega_{2}\left(\varphi_{1}\right) \Omega_{2}\left(\psi^{\prime}\right) .
$$

By Proposition 5.1, $\Omega_{1}\left(\varphi^{\prime}\right)=\Omega_{1}(\varphi)$. Since, by assumption, $\Omega_{i}(\psi)=\Omega_{i}\left(\psi^{\prime}\right)$ for $i=1$, 2 , we conclude that $\Omega_{2}\left(\varphi_{2}\right)=\Omega_{2}\left(\varphi_{2}^{\prime}\right)$ which we wanted to prove.

(i) Since $\psi$ is the kernel of $\varphi_{2}$, a sequence of proper computations $\varphi^{(\kappa)}$, $\kappa=0, \ldots, k$, of $M^{3}$ exists such that $\varphi^{(0)}=\varphi_{2}, \varphi^{(k)}=\psi$, and, for $\kappa=0, \ldots, k-1$, either (1) or (2) holds:

(1) $\varphi^{(\kappa)}=\hat{\varphi}_{1} \hat{\varphi}_{2} \hat{\varphi}_{3}$ and $\varphi^{(\kappa+1)}=\hat{\varphi}_{1} \hat{\varphi}_{3}$ where $\hat{\varphi}_{2}$ is a proper $(z, z)$-computation for some $z \in Q^{3}$.

(2) $\varphi^{(x)}=\hat{\varphi}_{1}\left[x_{1}, \hat{\varphi}_{2} \hat{\varphi}_{3}\right]$ and $\varphi^{(\kappa+1)}=\hat{\varphi}_{1}\left[x_{1}, \hat{\varphi}_{3}\right]$ where $\hat{\varphi}_{2}$ is a proper $(z, z)$ computation for some $z \in Q^{3}$.

By Properties ( $\left.\mathrm{S} 1^{\prime}\right)$ and $\left(\mathrm{S} 2^{\prime}\right)$ we deduce in both cases that $\Omega_{2}\left(\hat{\varphi}_{2}\right) \in\left\{0, x_{1}\right\}$ and therefore $T_{2}\left(\hat{\varphi}_{2}\right) \in\left\{\perp, x_{1}\right\}$. Therefore, by the reducedness of $M$,

$$
T_{2}\left(\hat{\varphi}_{2} \hat{\varphi}_{3}\right)=T_{2}\left(\hat{\varphi}_{3}\right)
$$

and hence also $T_{2}\left(\varphi^{(\kappa)}\right)=T_{2}\left(\varphi^{(\kappa+1)}\right)$ for all $\kappa$. Now statement (i) follows by induction on $\kappa$.

(ii) Now assume $q \notin \operatorname{Const}(M)$. Since $\psi$ is the kernel of $\varphi_{2}$, a sequence of computations $\varphi^{(\kappa)}, \kappa=0, \ldots, k$, of $M^{3}$ exists such that $\varphi^{(0)}=\varphi, \varphi^{(k)}=\tilde{\varphi}$, and, for $\kappa=0, \ldots, k-1$, either (1) or (2) above holds. Therefore, by Facts 2(i) and 3(i) from Section 3,

$$
\Omega_{2}\left(\varphi^{(\kappa)}\right)^{-1} \Omega_{1}\left(\varphi^{(\kappa)}\right)=\Omega_{2}\left(\varphi^{(\kappa+1)}\right)^{-1} \Omega_{1}\left(\varphi^{(\kappa+1)}\right) .
$$

By this, assertion (ii) follows with induction on $\kappa$.

Using Proposition 5.3 we are able to prove Proposition 5.2.

Proof of Proposition 5.2. First recall that since $M$ has Properties (F1) and (F2), $M$ also has Properties ( $\left.\mathrm{F0}^{\prime}\right)$, (S1), and (S2). Now assume $n:=\# Q$. Every kernel $\psi$ is a computation of $M^{3}$ of depth at most $2 \cdot n^{3}$. Therefore, $\Omega_{i}(\psi)=a+b \cdot x_{1}$ where

$$
a<(L+1)^{2 n^{3}} \cdot|M|^{2 n^{3}+1}
$$

and

$$
b<|M|^{2 n^{3}} .
$$


Moreover, there are at most $|M|^{3 \cdot(L+1)^{2 \cdot n^{3}}}$ different kernels. By the assumptions on the set $B, j \in \mathbb{N}$ and states $p_{1}, \ldots, p_{6} \in Q$ exist such that

$$
\begin{aligned}
& \left(p_{1}, t^{j}, u_{1}, p_{2}\right),\left(p_{2}, t, u_{2}, p_{3}\right),\left(p_{3}, t^{j}, u_{3}, p_{1}\right), \\
& \left(p_{1}, t^{j}, u_{4}, p\right),\left(q, t^{j}, u_{5}, p_{4}\right), \\
& \left(p_{4}, t^{j}, u_{6}, p_{5}\right),\left(p_{5}, t, u_{7}, p_{6}\right),\left(p_{6}, t^{j}, u_{8}, p_{4}\right) \in \delta
\end{aligned}
$$

for certain $u_{1}, \ldots, u_{8} \in T_{\Delta}\left(x_{1}\right) \cup\{\perp\}$. Let $u:=u_{1} u_{2} u_{3}$, let $\varphi_{-1}$ be a $\left(p_{2}, p_{3}\right)$-computation, and let $\varphi_{1}$ be a $\left(p_{5}, p_{6}\right)$-computation of $A$ for $t$ producing outputs $u_{2}$ and $u_{7}$, respectively. We proceed similarly as in the proof of Lemma 2.8 of [W4] where our Proposition 5.3 is the substitute of Weber's Lemma 2.10.

Case 1: $u \in\left\{\perp, x_{1}\right\}$. By Proposition 5.3(i), the cardinality of the set $\{T(\varphi) \mid \varphi(p, q)$ computation of $M$ for $t\}$ is bounded by the number of different kernels of $\left(z_{1}, z_{2}\right)$-computations for $t$ where $z_{1}:=\left\langle p_{2}, p, p_{5}\right\rangle$ and $z_{2}:=\left\langle p_{3}, q, p_{6}\right\rangle$. Hence, it is at most $|M|^{3 \cdot(L+1)^{2 n^{3}}}$. From this, statements (i) and (ii) follow.

Case 2: $u \notin\left\{\perp, x_{1}\right\}$. Especially, $q$ cannot be unique-sized since $M$ has Property (S0). Therefore, all $u_{i}$ contain an occurrence of $x_{1}$. By Proposition 5.3(iii), the output sizes of $(p, q)$-computations $\varphi$ and $\varphi^{\prime}$ of $A$ for $t$ are equal whenever $\Omega_{i}(\psi)=\Omega_{i}\left(\psi^{\prime}\right)$, $i=1,2$, where $\psi$ is the kernel of $\left\langle\varphi_{-1}, \varphi, \varphi_{1}\right\rangle$, and $\psi^{\prime}$ is the kernel of $\left\langle\varphi_{-1}, \varphi^{\prime}, \varphi_{1}\right\rangle$. By estimations (1) and (2), there are at most $(L+1)^{2 n^{3}} \cdot|M|^{2 n^{3}+1} \cdot|M|^{2 n^{3}}$ different output sizes $\Omega_{i}(\psi), i=1,2$. Hence

$$
\#\{\omega(s) \mid(p, t, s, q) \in \delta\} \leq(L+1)^{4 n^{3}} \cdot|M|^{8 n^{3}+2},
$$

which implies statement (i).

To prove statement (ii) consider $\bar{t}:=t^{2 j+1}$ and assume that $\Omega_{i}(\psi)=\Omega_{i}\left(\psi^{\prime}\right)$. By Proposition 5.3(iii), $\Omega(\varphi)=\Omega\left(\varphi^{\prime}\right)$. It remains to show that $T(\varphi)=T\left(\varphi^{\prime}\right)$ also. Let $v:=T(\varphi)$ and $v^{\prime}:=T\left(\varphi^{\prime}\right)$. By our assumptions,

$$
\begin{aligned}
& \left(p_{1}, \bar{t}, u, p_{1}\right), \\
& \left(p_{1}, \bar{t}, u_{4} v u_{5}, p_{4}\right),\left(p_{1}, \bar{t}, u_{4} v^{\prime} u_{5}, p_{4}\right), \\
& \left(p_{4}, \bar{t}, u_{6} u_{7} u_{8}, p_{4}\right) \in \delta,
\end{aligned}
$$

where $u \notin\left\{\perp, x_{1}\right\}$ and $\omega\left(u_{4} v u_{5}\right)=\omega\left(u_{4} v^{\prime} u_{5}\right)$. Hence, $u_{4} v u_{5}=u_{4} v^{\prime} u_{5}$, because $M$ has Property $\left(\mathrm{F} 0^{\prime}\right)$. Since $v, v^{\prime}$, and $u_{i}$ all contain occurrences of $x_{1}$ we can apply top and bottom cancellation to deduce that $v=v^{\prime}$.

We apply Propositions 5.1 and 5.2 to obtain the main theorems of this section.

Theorem 5.4. There is a constant $c>0$ such that, for every reduced FST $M$, the following three statements are equivalent:

(i) $M$ has Properties (S1) and (S2).

(ii) $\operatorname{sval}(M)<2^{2^{c \mid}|M| \cdot \log (I+1)}$.

(iii) $\operatorname{sval}(M)<\infty$. 
Theorem 5.5. There is a polynomial $P$ such that, for every reduced FST M, the following three statements are equivalent:

(i) $M$ has Properties (F1) and (F2).

(ii) $\operatorname{val}(M)<2^{2^{P(M)}}$.

(iii) $\operatorname{val}(M)<\infty$.

Proposition 3.4 now gives the following corollary:

Corollary 5.6. Assume $M$ is a reduced FST. Then $\operatorname{val}(M)<\infty$ iff $\operatorname{sval}(M)<\infty$ and $M$ has Property (F0).

Simple examples show that Properties (F1) and (F2) are independent. Moreover, modifying an example of [Se1] we find that, for some constant $c>0$, and infinite family of finite-valued FSTs $M$ of arbitrarily large size exists with $\operatorname{val}(M)>2^{2 c|M|}$.

We only prove Theorem 5.5. The proof of Theorem 5.4 is analogous: instead of using statements (ii) of the Propositions 5.1 and 5.2 statements (i) have to be employed.

Proof of 5.5. Assertion (ii) implies (iii). By Theorem 3.1(ii), assertion (iii) implies (i). Therefore, it remains to show that (i) implies (ii).

Assume $p, q \in Q$. Define

$T_{p}(t):=\{T(\varphi) \mid \varphi p$-computation of $M$ for $t\}$

and

$\operatorname{val}_{p}(t):=\# T_{p}(t) \quad$ for every $\quad t \in T_{\Sigma}$,

$T_{q, p}(t):=\{T(\varphi) \mid \varphi(q, p)$-computation of $M$ for $t\}$

and

$\operatorname{val}_{q, p}(t):=\# T_{q, p}(t) \quad$ for every proper $\quad t \in T_{\Sigma}\left(x_{1}\right)$.

Define a function $v\left({ }_{-}\right): \mathbb{N} \times \mathbb{N} \rightarrow \mathbb{N} \cup\{\infty\}$ such that $v(n, k)$ is the supremum of the numbers $\operatorname{val}(M)$ where $M$ is a reduced FST with one single final state, $|M| \leq n$, and with at most $k$ strong components such that $M$ has Properties (F1) and (F2).

Clearly, $n \cdot v(n, n)$ is an upper bound for the valuedness of a reduced finitevalued FST of size $n$. Therefore, it suffices to compute an upper bound for $v(n, k)$. We proceed by induction on $k^{\prime}$. We claim

$$
\begin{aligned}
& v(n, 1) \leq n \quad \text { for every } n>0, \\
& v(n, k) \leq 2^{2^{P(n)+c n \cdot(1+\log \log L)+(k-1) \cdot \log (L+1)}}
\end{aligned}
$$

for every $n>0$ and $k>1$ where $P^{\prime}$ is the polynomial given by Proposition 5.2 and $c$ is some constant $>0$. Clearly, inequalities (1) and (2) prove the stated implication. 
For a proof consider a reduced FST $M=\left(Q, \Sigma, \Delta, \delta,\left\{q_{\mathrm{F}}\right\}\right)$ having $k \geq 1$ strong components $Q_{1}, \ldots, Q_{k}$. Clearly, $q_{\mathrm{F}} \in Q_{1}$. Let $t \in T_{\Sigma}$. For some node $o \in O(t)$ let $t\left[x_{1} / o\right]$ denote the tree obtained from $t$ by replacing the subtree with root $o$ by $x_{1}$. First assume $k=1$. Let $o \in O(t)$ an arbitrary leaf of $t$ with label $a$. Then every accepting computation $\varphi$ can be factorized $\varphi=\psi \tau$ where, for some $p \in Q$, $\psi=\varphi\left[x_{1} / o\right]$ is a $\left(q_{\mathrm{F}}, p\right)$-computation for $t\left[x_{1} / o\right]$, and $\tau$ is a $p$-transition $(p, a, s, \varepsilon)$ for some $s$. Let $\varphi^{\prime}$ be another accepting computation of $M$ for $t$ where $\varphi^{\prime}(o)=$ $\varphi(o)=\tau$. By Proposition 5.1, $T\left(\varphi^{\prime}\left[x_{1} / o\right]\right)=T\left(\varphi\left[x_{1} / o\right]\right)$. Therefore, $T(\varphi)=T\left(\varphi^{\prime}\right)$ also. We conclude that the number of different possible outputs is bounded by the number of different transitions for some $a \in \Sigma_{0}$. This gives estimation (1).

Now consider $k>1$. For $t \in T_{\Sigma}$ and $o \in O(t)$ define

$\operatorname{ACC}_{t}(o):=\left\{q \in Q \mid\right.$ there is a $\left(q_{\mathrm{F}}, q\right)$-computation for $\left.t\left[x_{1} / o\right]\right\}$,

$\operatorname{DER}_{t}(o):=\{q \in Q \mid$ there is a $q$-computation for $t / o\}$,

and

$$
D_{t}(o):=\operatorname{ACC}_{t}(o) \cap \operatorname{DER}_{t}(o) .
$$

Observe that, by our definition, $D_{t}(o)$ is precisely the set of all states $q \in Q$ for which some accepting computation $\varphi$ exists where $\varphi(o)$ is a $q$-transition.

We decompose $t$ according to the first and latest occurrences of the sets of states. For $o \in O(t)$ define $\operatorname{succ}(o)$ as the lexically smallest node $\bar{o}$ such that:

- $o$ is a prefix of $\bar{o}$,

- $D_{t}(o)=D_{t}(\bar{o})$, and

- $D_{t}\left(\bar{o} o^{\prime}\right) \neq D_{t}(o)$ for every $o^{\prime} \neq \varepsilon$.

The set of designated nodes of $t$ is the smallest set $O \subseteq O(t)$ such that:

(i) $\varepsilon \in O$,

(ii) if $o \in O$, then $\operatorname{succ}(o) \in O$ also, and

(iii) if $o \in O$ and $\operatorname{succ}(o)=o$, then $o j \in O$ for all $j$.

Define $O_{1} \subseteq O$ as the set of nodes in $O$ with $\operatorname{succ}(o)=o$. We have

$$
\#\left(O \backslash O_{1}\right) \leq \# O_{1} \quad \text { and } \quad \# O_{1}<(L+1)^{2^{n-1}} .
$$

The type of a node $o \in O$, type $(o) \in\{1, \ldots, L\}^{*}$, describes the sequence of "decisions" made at nodes from $O_{1}$ to reach $o$. It is inductively defined as follows:

(i) type $(\varepsilon):=\varepsilon$,

(ii) type(succ(o)) $=\operatorname{type}(o)$, and

(iii) if $o=\operatorname{succ}(o)$, then type $(o j):=\operatorname{type}(o) \cdot j$.

In the next section we need the types of nodes from $O$ to find a fixed finite set of specifications of outputs for input trees. For the moment, consider just one input tree $t$. We classify the accepting computations of $M$ for $t$ according to set $O$. 
We have

$$
\begin{aligned}
& T_{M}(t)=\bigcup_{\substack{o \in O \backslash O_{1} \\
p \in Q_{1} \\
q \notin Q_{1}}} T_{q_{F}, p}\left(t\left[x_{1} / o\right]\right) T_{p, q}\left(t / o\left[x_{1} / \operatorname{succ}(o)\right]\right) T_{q}(t / \operatorname{succ}(o))
\end{aligned}
$$

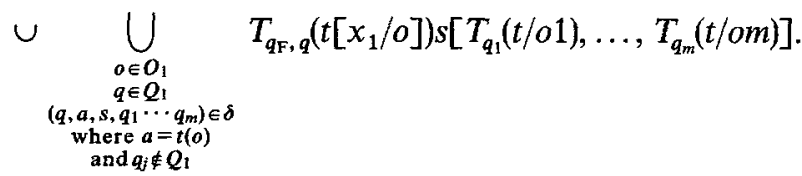

Proposition 5.1 applied to $t\left[x_{1} / o\right]$ yields that $\# T_{M}\left(t\left[x_{1} / o\right]\right)=1$. Furthermore, we apply Proposition 5.2 to $t / o\left[x_{1} / \operatorname{succ}(o)\right]$ with $B=D_{t}(o)$ (which, by definition, equals $\left.D_{t}(\operatorname{succ}(o))\right)$ and obtain

$$
\begin{aligned}
& \operatorname{val}_{M}(t) \leq \sum_{\substack{o \in O O O_{1} \\
q \notin q_{1}}} 2^{2 p^{\prime(n)}} \cdot n \cdot \operatorname{val}_{q}(t / \operatorname{succ}(o)) \\
& +\sum_{\substack{o \in O_{1} \\
\left(q, a, s, q_{1}, q_{m}\right) \in \delta \\
\text { where }=t+t(t) \\
\text { and } q \in Q_{1}, q_{j} \notin Q_{1}}} \operatorname{val}_{q_{1}}(t / o 1) \cdots \cdots \operatorname{val}_{q_{m}}(t / o m),
\end{aligned}
$$

where $P^{\prime}$ is a polynomial chosen according to Proposition 5.2. It follows that

$$
\begin{aligned}
& v(n, k) \leq(L+1)^{2^{n-1}}\left[2^{2^{P^{(n)}}} \cdot n^{2} \cdot v(n, k-1)+n \cdot v(n, k-1)^{L}\right] \\
& \leq 2^{2^{P(n)+c^{\prime}(1+\log \log L)} \cdot v(n, k-1)^{L}}
\end{aligned}
$$

for some constant $c^{\prime}>0$. Hence by inequality (1),

$\log \log v(n, k) \leq P^{\prime}(n)+c n(1+\log \log L)+(k-1) \log (L+1)$

Using Theorem 4.1 we conclude:

\section{Theorem 5.7.}

(i) It is decidable in polynomial space whether or not an FST $M$ is finite size-valued.

(ii) It is decidable in nondeterministic polynomial time whether an FST M is not finite-valued.

Proof. Theorems 5.4 and 4.1(i) for statement (i); Theorems 5.5 and 4.1(ii) for statement (ii).

\section{Decomposing Finite-Valued Tree Transducers}

In this section we prove that it can be decided whether or not the translation of an FST is included in the translation of a finite-valued FST $M^{\prime}$ (Theorem 6.4). Actually, our Theorem 1.5, Property (F0'), and Proposition 5.3 allow us to 
generalize the corresponding constructions of [W2]. Therefore, we proceed as follows. In Theorem 6.1 we show that this inclusion problem is solvable provided $T\left(M^{\prime}\right)$ is a finite union of the translations of single-valued FSTs. In Theorem 6.2 we show that every finite-valued FST $M^{\prime}$ can be effectively decomposed into a finite union of single-valued FSTs. Together, this yields the desired result.

For size-valuedness, the theorems corresponding to Theorems 6.1 and 6.2 hold as well. We do no state them explicitly but refer to them as Theorems 6.1S and 6.2S. Especially, we are able to decide whether or not two finite size-valued transducers $M$ and $M^{\prime}$ are equivalent with respect to the sets of output sizes, i.e., whether $\Omega(M)=\Omega\left(M^{\prime}\right)$.

Theorem 6.1. Assume $M$ is an FST and $M_{1}, \ldots, M_{K}$ are single-valued FSTs such that $L(M) \subseteq L\left(M_{j}\right)$ for all $j=1, \ldots, K$. Assume the number of states of any of these transducers is bounded by $n$. Then $T(M) \nsubseteq \bigcup_{j=1}^{k} t\left(M_{j}\right)$ iff there is a pair $(t, s) \in T(M) \backslash \bigcup_{j=1}^{K} T\left(M_{j}\right)$ such that $\operatorname{depth}(t) \leq 3 \cdot(2 K) ! \cdot n^{K+1}$.

Proof. Assume $t \in T_{\Sigma}, \varphi_{0}$ is an accepting computation of $M$ for $t$. Since $L(M) \subseteq$ $L\left(M_{j}\right)$ for $j=1, \ldots, K$, there are accepting computations $\varphi_{j}$ of $M_{j}$ for $t$ for all $j$. Define $s_{j}:=T\left(\varphi_{j}\right)$ for $j=0, \ldots, K$. Assume $t$ is chosen of minimal size such that $s_{0} \neq s_{j}$ for all $j=1, \ldots, K$, but depth $(t)>N \cdot n^{K+1}$ where $N=3 \cdot(2 K) !$. Then there are states $p_{0}, \ldots, p_{K} \in Q$, a factorization of $t=t_{0} \cdots t_{N}$ into proper trees $t_{j} \in T_{\Sigma}\left(x_{1}\right)$, and corresponding factorizations $\varphi_{\kappa}=\varphi_{\kappa, 0} \cdots \varphi_{\kappa, N}$ such that $\varphi_{\kappa, j}$ is a $\left(p_{\kappa}, p_{\kappa}\right)$ computation for all $\kappa=0, \ldots, K$ and $j=1, \ldots, N-1$. Define $s_{\kappa, j}:=T\left(\varphi_{\kappa, j}\right)$, $\kappa=0, \ldots, K, j=0, \ldots, N$. By the minimality of $t$,

$$
\begin{aligned}
& \forall 0<i<j \leq N, \quad \exists \kappa \in\{1, \ldots, K\}, \\
& \quad s_{0,0} \cdots s_{0, i-1} s_{0, j} \cdots s_{0, N}=s_{\kappa, 0} \cdots s_{\kappa, i-1} s_{\kappa, j} \cdots s_{\kappa, N} .
\end{aligned}
$$

By Theorem 1.5(ii), this implies

$$
T\left(\varphi_{0}\right)=s_{0,0} \cdots s_{0, N}=s_{\kappa, 0} \cdots s_{\kappa, N}=T\left(\varphi_{\kappa}\right) \quad \text { for some } \quad \kappa \in\{1, \ldots, K\},
$$

a contradiction.

Theorem 6.2 (Decomposition of Finite-Valued FSTs). For every finitely valued

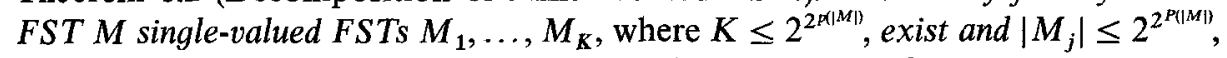
$j=1, \ldots, K$, for some polynomial $P$ independent of $M$ such that

$$
T(M)=T\left(M_{1}\right) \cup \cdots \cup T\left(M_{K}\right) .
$$

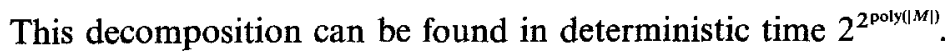

Proof. Assume $M=\left(Q, \Sigma, \Delta_{\perp}, \delta, Q_{\mathrm{F}}\right)$. Without loss of generality, we may assume $M$ is reduced and that $Q_{\mathrm{F}}=\left\{q_{\mathrm{F}}\right\}$. Recall that $M_{q}$ denotes the FST $\left(Q, \Sigma, \Delta_{\perp}, \delta,\{q\}\right)$. Note that $\operatorname{val}\left(M_{q}\right)<\infty$.

Assume $t \in T_{\Sigma}$ and $O \subseteq O(t)$ is the set of designated nodes of $t$ as defined in 
the proof of Theorem 5.5. We classify the accepting computations of $M$ for $t$ according to $O$. First we make the following observation:

Observation 6.3. Assume $\varphi$ is a q-computation for t. If $\infty>\operatorname{val}\left(M_{q}\right)>1$, then there is exactly one $o \in O(t)$ such that:

(i) $\varphi(o)$ is a p-transition for some $p \in Q$ with $p \rightarrow_{M} q$.

(ii) $o$ is of maximal length, i.e., if $\varphi\left(o_{1}\right)$ is a $p^{\prime}$-transition with $p^{\prime} \rightarrow_{M} q$, then $o_{1}=\varepsilon$.

Proof. Assume the assertion is not true. Then there is a node $o \in O(t)$ and $j_{1} \neq j_{2}$ such that both $p_{1} \rightarrow_{M} q$ and $p_{2} \rightarrow_{M} q$ where $\varphi\left(o j_{i}\right)$ is a $p_{i}$-transition. From Proposition 5.1, applied to $q, p_{1}$, and $t\left[x_{1} / o j_{1}\right]$, we get $\operatorname{val}\left(M_{p_{2}}\right)=1$ and, therefore, $\operatorname{val}\left(M_{q}\right)=1$ since $p_{2} \rightarrow_{M} q$ and $M$ is reduced. This is a contradiction.

For convenience we denote the unique node $o$ of Observation 6.3 by $\pi(\varphi)$. Assume $\varphi$ is $q$-computation of $M$ for $t$. To $\varphi$ we attach a (finite) set of specifications $\Gamma(\varphi)$. A specification $\gamma$ is accepting iff $\gamma \in \Gamma(\varphi)$ for some accepting computation $\varphi$. If $\operatorname{val}\left(M_{q}\right)=1, \Gamma(\varphi):=\{q\}$. If $\operatorname{val}\left(M_{q}\right)>1$ we distinguish two cases.

Case 1: $\pi(\varphi) \in O_{1}$. Assume $h:=\operatorname{type}(\pi(\varphi))$, and let $t=t_{1} t_{2}$ where $t_{1}:=t\left[x_{1} / \pi(\varphi)\right]$. Assume $\varphi=\varphi_{0} \varphi^{\prime}$ is the corresponding decomposition of $\varphi$ with $\varphi^{\prime}=\tau\left(\varphi_{1}, \ldots, \varphi_{k}\right)$. Then $\langle q, h, \tau\rangle\left(\gamma_{1}, \ldots, \gamma_{k}\right) \in \Gamma(\varphi)$ for every $\gamma_{j} \in \Gamma\left(\varphi_{j}\right), j=1, \ldots, k$.

Case 2. Case 1 does not hold. Then a node $o \in O \backslash O_{1}$ of some type $h$ exists such that $o$ is a prefix of $\pi(\varphi)$ but $\operatorname{succ}(o)$ is not a prefix of $\pi(\varphi)$. Assume $t=t_{1} t_{2} t_{3}$ for proper $t_{1}, t_{2} \in T_{\Sigma}\left(x_{1}\right)$ such that $t_{1} / o=x_{1}$; and $t_{1} t_{2} / \operatorname{succ}(o)=x_{1}$; and let $\varphi=$ $\varphi_{0} \psi_{0} \varphi^{\prime}$ be the corresponding decomposition of $\varphi$ where $\psi_{0}$ is a proper $\left(p_{0}, q_{0}\right)$ computation and $\varphi^{\prime}=\tau\left(\varphi_{1}, \ldots, \varphi_{k}\right)$.

Consider states $p_{-1}, p_{1}, q_{-1}, q_{1} \in Q$ such that $\left(p_{i}, q_{i}\right)$-computations $\psi_{i}$ for $t_{2}$ exist for $i \in\{-1,+1\}$ where

$$
\begin{aligned}
& \left(q_{-1}, u, s_{1}, r_{1}\right),\left(q_{0}, u, s_{2}, r_{2}\right),\left(q_{1}, u, s_{3}, r_{2}\right), \\
& \left(r_{1}, v, s_{4}, p_{-1}\right),\left(r_{1}, v, s_{5}, p_{0}\right),\left(r_{2}, v, s_{6}, p_{1}\right) \in \delta
\end{aligned}
$$

for some proper trees $u$, and $v$, states $r_{1}, r_{2} \in Q$, and $s_{1}, \ldots, s_{6} \in T_{\Delta}\left(x_{1}\right)$. Since $D_{t}(o)=D_{t}(\operatorname{succ}(o))$ such a choice always is possible with $u=v=t_{2}^{j}$ for some $j$ (see the proof of Proposition 5.2).

Finally, let $\psi$ be the kernel of $\left\langle\psi_{-1}, \psi_{0}, \psi_{1}\right\rangle$. Then $\langle q, h, \psi, \tau\rangle\left(\gamma_{1}, \ldots, \gamma_{k}\right) \in$ $\Gamma(\varphi)$ for every $\gamma_{j} \in \Gamma\left(\varphi_{j}\right), j=1, \ldots, k$.

We claim:

(1) The set $\Gamma:=\bigcup\{\Gamma(\varphi) \mid \varphi$ accepting computation of $M\}$ has cardinality at most $2^{2^{P(M)}}$ for some polynomial $P$ independent of $M$.

(2) If $\varphi^{(1)}$ and $\varphi^{(2)}$ are accepting computations of $M$ for the same tree $t$ and $\Gamma\left(\varphi^{(1)}\right) \cap \Gamma\left(\varphi^{(2)}\right) \neq \varnothing$, then $T\left(\varphi^{(1)}\right)=T\left(\varphi^{(2)}\right)$.

For a proof of (1) observe that a type $h \in\{1, \ldots, L\}^{*}$ is a word of length at most $2^{n}-1$. Moreover, recall that there are less than $|M|^{3 \cdot(L+1)^{2 n^{3}}}$ kernels (where 
$n=\# Q$ ). Therefore, the signature to denote the trees from $\Gamma$ has cardinality at most

$$
n \cdot(L+1)^{2^{n}-1} \cdot|M|^{3 \cdot(L+1)^{2 n^{3}}} \leq 2^{2^{P_{1}(|M|)}}
$$

for some polynomial $P_{1}$ independent of $M$. Furthermore, assume $\gamma=A\left(\gamma_{1}, \ldots, \gamma_{m}\right)$ is a specification of an accepting computation $\varphi$ of an FST $M$ with $k$ strong components. Then $\gamma_{j}$ is a specification of an accepting computation of an FST $M_{j}$ with at most $k-1$ strong components. Thus, $\operatorname{depth}(\gamma) \leq k \leq n$. Since the rank of the signature of $\Gamma$ is at most $L$, claim (1) follows.

Now consider claim (2). Let $t \in T_{\Sigma}, O \subseteq O(t)$ be the set of designated nodes of $t$ and let $\varphi^{(1)}, \varphi^{(2)}$ be $q$-computations of $M$ for $t$ such that $\Gamma\left(\varphi^{(1)}\right) \cap \Gamma\left(\varphi^{(2)}\right) \neq \varnothing$. Assume $\gamma$ is in both $\Gamma\left(\varphi^{(1)}\right)$ and $\Gamma\left(\varphi^{(2)}\right)$. We deduce that $T\left(\varphi^{(1)}\right)=T\left(\varphi^{(2)}\right)$ by induction on the structure of $\gamma$. If $\gamma=q$, then $M_{q}$ is single-valued. Hence, $T\left(\varphi^{(1)}\right)=T\left(\varphi^{(2)}\right)$. If $\gamma \notin Q$, then we have to distinguish two cases.

Case 1: $\gamma=\langle q, h, \tau\rangle\left(\gamma_{1}, \ldots, \gamma_{k}\right)$. Then $o \in O_{1}$ of type $h$ exists such that $t=t_{1} t_{2}$ for proper tree $t_{1} \in T_{\Sigma}\left(x_{1}\right)$ where $t_{1} / o=x_{1}$. Let $\varphi^{(i)}=\varphi_{0}^{(i)} \bar{\varphi}^{(i)}$ be the corresponding decompositions of $\varphi^{(i)}, i=1,2$, with $\bar{\varphi}^{(i)}=\tau\left(\varphi_{1}^{(i)}, \ldots, \varphi_{k}^{(i)}\right)$ such that $\tau=\left(p, a, u, q_{1} \cdots q_{k}\right) \in \delta$ where $q$ and $p$ are connected but $q$ is not reachable from any of the $q_{j}$, and $\gamma_{j} \in \Gamma\left(\varphi_{j}^{(i)}\right)$ for all $j$ and $i=1,2$. We show that $T\left(\varphi_{0}^{(1)}\right)=T\left(\varphi_{0}^{(2)}\right)$ and $T\left(\bar{\varphi}^{(1)}\right)=T\left(\bar{\varphi}^{(2)}\right)$. Since both $\varphi_{0}^{(1)}$ and $\varphi_{0}^{(2)}$ are $(q, p)$-computations of $M$ for $t_{1}$, Proposition 5.1 implies that $T\left(\varphi_{0}^{(1)}\right)=T\left(\varphi_{0}^{(2)}\right)$.

Moreover, by inductive assumption, $T\left(\varphi_{j}^{(1)}\right)=T\left(\varphi_{j}^{(2)}\right)$ for all $j=1, \ldots, k$. Therefore,

$$
T\left(\bar{\varphi}^{(1)}\right)=u\left[T\left(\varphi_{1}^{(1)}\right), \ldots, T\left(\varphi_{k}^{(1)}\right)\right]=u\left[T\left(\varphi_{1}^{(2)}\right), \ldots, T\left(\varphi_{k}^{(2)}\right)\right]=T\left(\bar{\varphi}^{(2)}\right),
$$

which finishes the proof in this case.

Case 2: $\gamma=\langle q, h, \psi, \tau\rangle\left(\gamma_{1}, \ldots, \gamma_{m}\right)$. Then $o \in O \backslash O_{1}$ of type $h$ exists such that $t=t_{1} t_{2} t_{3}$ for proper trees $t_{1}, t_{2}$ where $t_{1}:=t\left[x_{1} / o\right]$ and $t_{1} t_{2} / \operatorname{succ}(o)=x_{1}$. Consider the corresponding decompositions $\varphi^{(i)}=\varphi_{0}^{(i)} \psi_{0}^{(i)} \bar{\varphi}^{(i)}$ of $\varphi^{(i)}, i=1,2$, where $\psi_{0}^{(i)}$ are proper $\left(p_{0}^{(i)}, q_{0}^{(i)}\right)$-computations for states $p_{0}^{(i)}, q_{0}^{(i)}$ such that $q \leftrightarrow_{M} p_{0}^{(i)}$ but not $q_{0}^{(i)} \rightarrow_{M} q$. Moreover, $\bar{\varphi}^{(i)}=\tau\left(\varphi_{1}^{(i)}, \ldots, \varphi_{k}^{(i)}\right)$ with $\gamma_{j} \in \Gamma\left(\varphi_{j}^{(i)}\right)$ for all $j$ and $i=1,2$.

We show that $T\left(\varphi_{0}^{(1)}\right)=T\left(\varphi_{0}^{(2)}\right), T\left(\psi_{0}^{(1)}\right)=T\left(\psi_{0}^{(2)}\right)$, and $T\left(\bar{\varphi}^{(1)}\right)=T\left(\bar{\varphi}^{(2)}\right)$. Since $\gamma \in \Gamma\left(\varphi^{(1)}\right) \cap \Gamma\left(\varphi^{(2)}\right),\left(p_{i}^{(v)}, q_{i}^{(v)}\right)$-computations $\psi_{i}^{(v)}$ for $t_{2}$ for $i \in\{-1,+1\}$ also exist such that $\psi$ is the kernel of $\left\langle\psi_{-1}^{(v)}, \psi_{0}^{(v)}, \psi_{+1}^{(v)}\right\rangle$ for $v=1,2$. Therefore, in particular,

(i) $p_{i}^{(1)}=p_{i}^{(2)}=: p_{i} \quad$ and $\quad q_{i}^{(1)}=q_{i}^{(2)}=: q_{i} \quad$ for $\quad i=-1,0,1$.

Moreover,

(ii) $\left(r_{1}, u, s_{1}, p_{-1}\right),\left(q_{-1}, v, s_{2}, r_{1}\right)$,

$$
\begin{aligned}
& \left(r_{1}, u, s_{3}, p_{0}\right),\left(q_{0}, v, s_{4}, r_{2}\right), \\
& \left(r_{2}, u, s_{5}, p_{1}\right)\left(q_{1}, v, s_{6}, r_{2}\right) \in \delta
\end{aligned}
$$


for proper $u, v$, and certain $r_{1}, r_{2} \in Q, s_{1}, \ldots, s_{6} \in T_{\Delta}\left(x_{1}\right)$. By (i), both $\varphi_{0}^{(1)}$ and $\varphi_{0}^{(2)}$ are $\left(q, p_{0}\right)$-computations of $M$ for $t_{1}$. Since $p_{0}$ is connected with $q, T\left(\varphi_{0}^{(1)}\right)=T\left(\varphi_{0}^{(2)}\right)$ by Proposition 5.1.

Since $\psi=\mathbf{c}\left\langle\psi_{-1}^{(v)}, \psi_{0}^{(v)}, \psi_{+1}^{(v)}\right\rangle$ for $v=1,2$, we can apply Proposition 5.3 to the tree $t^{\prime}:=u t_{2} v$ where states $p, q$ and computations $\varphi_{2}, \varphi_{2}^{\prime}$ of $M^{3}$ in the assumption are $r_{1}, r_{2}$, and $\left\langle\psi_{-1}^{(i)}, \psi_{0}^{(i)}, \psi_{1}^{(i)}\right\rangle, i=1,2$, respectively. If $\Omega\left(\psi_{-1}^{(1)}\right) \in\left\{0, x_{1}\right\}$, then $\Omega\left(\psi_{-1}^{(2)}\right) \in\left\{0, x_{1}\right\}$ also, since $p_{-1}$ and $q_{-1}$ are connected. Therefore, by Proposition 5.3(i), $T\left(\psi_{0}^{(1)}\right)=T_{2}(\psi)=T\left(\psi_{0}^{(2)}\right)$. Otherwise, we apply Property $\left(\mathrm{F}^{\prime}\right)$ to $t^{\prime}$. By our assumptions,

$$
\left(r_{1}, t^{\prime}, s_{1}^{\prime}, r_{1}\right),\left(r_{1}, t^{\prime}, s_{2}^{\prime}, r_{2}\right),\left(r_{1}, t^{\prime}, s_{3}^{\prime}, r_{2}\right),\left(r_{2}, t^{\prime}, s_{4}^{\prime}, r_{2}\right) \in \delta,
$$

where

$$
s_{1}^{\prime}:=s_{1} T\left(\psi_{-1}^{(1)}\right) s_{2} \notin\left\{0, x_{1}\right\},
$$

and

$$
\begin{aligned}
& s_{2}^{\prime}:=s_{3} T\left(\psi_{0}^{(1)}\right) s_{4}, \\
& s_{3}^{\prime}:=s_{3} T\left(\psi_{0}^{(2)}\right) s_{4}, \\
& s_{4}^{\prime}:=s_{5} T\left(\psi_{1}^{(1)}\right) s_{6} .
\end{aligned}
$$

Proposition 5.3(iii) for $t^{\prime}$ gives $\Omega\left(\psi_{0}^{(1)}\right)=\Omega\left(\psi_{0}^{(2)}\right)$ and, therefore, $\omega\left(s_{2}^{\prime}\right)=\omega\left(s_{3}^{\prime}\right)$. Thus Property $\left(\mathrm{FO}^{\prime}\right)$ gives $s_{2}^{\prime}=s_{3}^{\prime}$. Since $s_{2}^{\prime}$ contains an occurrence of $x_{1}$, we deduce by top and bottom cancellation that $T\left(\psi_{0}^{(1)}\right)=T\left(\psi_{0}^{(2)}\right)$. fore,

Finally, by inductive assumption, $T\left(\varphi_{j}^{(1)}\right)=T\left(\varphi_{j}^{(2)}\right)$ for all $j=1, \ldots, k$. There-

$$
T\left(\bar{\varphi}^{(1)}\right)=u\left[T\left(\varphi_{1}^{(1)}\right), \ldots, T\left(\varphi_{k}^{(1)}\right)\right]=u\left[T\left(\varphi_{1}^{(2)}\right), \ldots, T\left(\varphi_{k}^{(2)}\right)\right]=T\left(\bar{\varphi}^{(2)}\right),
$$

where $u$ is the output pattern of $\tau$. This completes the proof of claim (2).

Note that in order to prove equality of outputs in Case 2 by means of Proposition 5.3, it would have been sufficient to encode states $\left\langle p_{-1}, p_{0}, p_{1}\right\rangle$, $\left\langle q_{-1}, q_{0}, q_{1}\right\rangle$, and the outputs of the components of kernel $\psi$ (or, in the case of size-valuedness, the sizes of the outputs). This leads to a (slightly) smaller set of specifications. In order to make our constructions simpler we did not do that.

For every specification $\gamma$, we construct an FST $M_{y}$ such that $T\left(M_{\gamma}\right)$ consists of all $(t, s) \in T_{\Sigma} \times T_{\Delta}$ such that $s=T(\varphi)$ for some computation $\varphi$ of $M$ for $t$ with $\gamma \in \Gamma(\varphi)$. Although much more involved, the construction is a generalization of the corresponding construction of [W2] to tree transducers. According to claim (2) above, the cardinality of $T_{M_{\gamma}}(t)$ is at most 1 for every $t \in T_{\Sigma}$. Therefore, $M_{\gamma}$ is single-valued. For every accepting computation $\varphi$ of $M, \Gamma(\varphi) \neq \varnothing$. Hence for all $t \in T_{\Sigma}, \bigcup_{y \in \Gamma} T_{M_{\gamma}}(t)=T_{M}(t)$. Therefore, $T(M)=\bigcup_{y \in \Gamma} T\left(M_{\gamma}\right)$.

It remains to give a construction for $M_{\gamma}$. We proceed in four steps. We start by constructing an FTA $M_{1}$ which on every input tree $t \in T_{\Sigma}$ computes the sets $D_{t}(r), r \in O(t)$. The states of $M_{1}$ have two components. In the second component $M_{1}$ computes the set of derivable states. Conceptually, this computation is done in a bottom-up fashion. Opposed to that, the first component propagates informa- 
tion top-down. It collects the subsets of states from the second component which in fact are accessible from the root.

To implement this, let $M_{1}=\left(Q_{1}, \Sigma, \Sigma, \delta_{1}, Q_{1, \mathrm{~F}}\right)$ be the FTA with

$$
\begin{aligned}
& Q_{1}:=\{\langle D, B\rangle \mid D \subseteq B \subseteq Q\}, \\
& Q_{1, \mathrm{~F}}:=\left\{\langle D, B\rangle \in Q_{1} \mid D=B \cap Q_{\mathrm{F}}\right\},
\end{aligned}
$$

and

$\left(\langle D, B\rangle, a, a,\left\langle D_{1}, B_{1}\right\rangle \cdots\left\langle D_{k}, B_{k}\right\rangle\right) \in \delta_{1} \quad$ iff

- $B=\left\{q \in Q \mid \exists\left(q, a, s, q_{1} \cdots q_{k}\right) \in \delta: \forall j: q_{j} \in B_{j}\right\}$ and

- $D_{j}=\left\{p \in B_{j} \mid \exists\left(q, a, s, q_{1} \cdots q_{k}\right) \in \delta: q \in D, q_{j}=p\right.$ and $\left.\forall j^{\prime} \neq j: q_{j^{\prime}} \in B_{j^{\prime}}\right\}$.

Clearly, \# $Q_{1} \leq 3^{n} ;|M| \leq 3^{|M|}$ and $M_{1}$ can be constructed in time $2^{\text {polyt( }|M|)}$.

Fact 6. Let $\varphi$ be an accepting computation of $M_{1}$ for some $t \in T_{\Sigma}$. Then, for every $r \in O(t), \varphi(r)$ is a $\langle D, B\rangle$-transition iff $B=\operatorname{DER}_{t}(r)$ and $D=D_{t}(r)$.

We use $M_{1}$ to construct an FTA $M_{2}$ which for every input tree $t$ computes the subset $O \subseteq O(t)$ of designated nodes of $t$ together with the corresponding types. On input tree $t, M_{2}$ conceptually runs top-down from the root to the leaves. Besides simulating $M_{1}$ in one component of its state we allow $M_{2}$ to have four more components. One component contains a flag from $\{0,1,2,3\} .0,1,2$, or 3 indicate that $M_{2}$ presently processes a prefix of some node of $t$ in $O$ which is not in $O$ itself, a node from $O_{1}$, a node from $O \backslash O_{1}$, or a node which is never a prefix of some node in $O$. In the second component $M_{2}$ computes the type of node in $O$. The third component holds a set $\sigma$ of sets of states of $M$ where we collect sets $D_{t}(r), r \in O$, already found. $M_{2}$ has to verify that these sets of states do not occur any more. The fourth component holds a set $D$ of states of which a leftmost latest occurrence is sought. $D$ is not added to $\sigma$ before this occurrence has been found.

Thus, let $M_{2}=\left(Q_{2}, \Sigma, \Sigma, \delta_{2}, Q_{2, \mathrm{~F}}\right)$ be the FTA with

$$
Q_{2}:=\{0,1,2,3\} \times T \times 2^{2^{Q}} \times 2^{Q} \times Q_{1},
$$

where $T:=\left\{w \in\{1, \ldots, L\}^{*}|| w \mid \leq 2^{n}-1\right\}$, with $n=\# Q$,

$$
Q_{2, \mathrm{~F}}:=\left\{\langle b, \varepsilon, \varnothing, D,\langle D, B\rangle\rangle \mid b \in\{1,2\},\langle D, B\rangle \in Q_{1, \mathrm{~F}}\right\},
$$

and

$$
\delta_{2}:=\delta_{21} \cup \delta_{22} \cup \delta_{23},
$$

where the sets of transitions $\delta_{2 i}$ are defined as follows:

$\delta_{21}$ consists of all transitions

$$
\left(\langle 3, h, \sigma, \varnothing,\langle D, B\rangle\rangle, a, a,\left\langle 3, h, \sigma, \varnothing,\left\langle D_{1}, B_{1}\right\rangle\right\rangle \cdots\left\langle 3, h, \sigma, \varnothing,\left\langle D_{k}, B_{k}\right\rangle\right\rangle\right)
$$


where

- $\left(\langle D, B\rangle, a, a,\left\langle D_{1}, B_{1}\right\rangle \cdots\left\langle D_{k}, B_{k}\right\rangle\right) \in \delta_{1}$ and

- $D, D_{1}, \ldots, D_{k} \notin \sigma$.

$\delta_{22}$ consists of all transitions

$\left(\langle 1, h, \sigma, D,\langle D, B\rangle\rangle, a, a,\left\langle b_{1}, h \cdot 1, \sigma^{\prime}, D_{1},\left\langle D_{1}, B_{1}\right\rangle\right\rangle \cdots\right.$

$\left.\left\langle b_{k}, h \cdot k, \sigma^{\prime}, D_{k},\left\langle D_{k}, B_{k}\right\rangle\right\rangle\right)$

with $\sigma^{\prime}=\sigma \cup\{\mathrm{D}\}$ and $b_{j} \in\{1,2\}$ where

- $\left(\langle D, B\rangle, a, a,\left\langle D_{1}, B_{1}\right\rangle \cdots\left\langle D_{k}, B_{k}\right\rangle\right) \in \delta_{1}$ and

- $D, D_{1}, \ldots, D_{k} \notin \sigma$.

$\delta_{23}$ consists of all transitions

$\left(\left\langle b, h, \sigma, D^{\prime},\langle D, B\rangle\right\rangle, a, a,\left\langle 3, h, \sigma^{\prime}, \varnothing,\left\langle D_{1}, B_{1}\right\rangle\right\rangle \cdots\left\langle 3, h, \sigma^{\prime}, \varnothing,\left\langle D_{j-1}, B_{j-1}\right\rangle\right\rangle\right.$
$\left.\left\langle b^{\prime}, h, \sigma, D^{\prime},\left\langle D_{j}, B_{j}\right\rangle\right\rangle\left\langle 3, h, \sigma, \varnothing,\left\langle D_{j+1}, B_{j+1}\right\rangle\right\rangle \cdots\left\langle 3, h, \sigma, \varnothing,\left\langle D_{k}, B_{k}\right\rangle\right\rangle\right)$

with $k>0, \sigma^{\prime}=\sigma \cup\left\{D^{\prime}\right\}, b \in\{0,2\}$ and $b^{\prime} \in\{0,1,2\}$ where

- $\left(\langle D, B\rangle, a, a,\left\langle D_{1}, B_{1}\right\rangle \cdots\left\langle D_{k}, B_{k}\right\rangle\right) \in \delta_{1}$,

- $D^{\prime}, D, D_{1}, \ldots, D_{k} \notin \sigma$, and

- if $b^{\prime} \in\{1,2\}$, then $D^{\prime}=D_{j}$.

The first set of transitions describes how $M_{2}$ processes nodes outside the set of prefixes of designated nodes $O$; the second and third sets give the behavior at nodes from $O_{1}$ and at prefixes from nodes in $O$ which are themselves not in $O_{1}$, respectively. In particular, transitions chosen at leaves are either from the first or the second set. Clearly, $\left|M_{2}\right| \leq 2^{2^{c \cdot|M|}}$ for some constant $c>0$, and $M_{2}$ can be

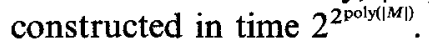

Fact 7. Let $\varphi$ be an accepting computation of $M_{2}$ for some $t \in T_{\Sigma}, r \in O(t)$, and $\varphi(r)$ is $a\left\langle b, h, \sigma, D^{\prime},\langle D, B\rangle\right\rangle$-transition. Then:

(i) $D=D_{t}(r)$.

(ii) If $b=0$, then $r$ is a prefix of some node in $O$ but $r \notin O$.

If $b=1$, then $r \in O_{1}$.

If $b=2$, then $r \in O \backslash O_{1}$.

If $b=3$, then $r$ is not a prefix of any node in $O$.

(iii) If $b \in\{1,2\}$, then type $(r)=h$.

Finally, we need an auxiliary FST $M_{3}$ which computes the kernel of a guessed proper computation $\varphi$ of $M^{3}$ and, while doing so, produces $T_{2}(\varphi)$ as output. Similar to $M_{2}$, it memorizes leftmost latest occurrences-but now of states of $M^{3}$. Therefore, it again has one component holding a flag from $\{0,1,2,3\}$, one component in which the kernel is computed, one component holding the set of forbidden states (of $M^{3}$ ), one component for the state (of $M^{3}$ ) actually processed, and one component in which a computation of $M^{3}$ is simulated. 
We are only interested in the behavior of $M_{3}$ on proper trees from $T_{\Sigma}\left(x_{1}\right)$. For simplicity we assume the labels of nodes on the (unique) path to leaf $x_{1}$ already contain some tag $j$ indicating which subtree at this node contains $x_{1}$. Such trees are said to have a designated branch. Formally, let $\bar{\Sigma}:=\Sigma \cup\{*\} \cup\{(a, j) \mid a \in \Sigma$, $1 \leq j \leq \rho(a)\}$ where the rank of $*$ is 0 and the rank of $(a, j)$ equals the rank of $a$. $t \in T_{\bar{\Sigma}}$ has a designated branch $r$ iff the following three properties hold:

(i) $t(r)=*$,

(ii) for every prefix $r^{\prime} j$ of $r, t\left(r^{\prime}\right)=(a, j)$ for some $a \in \Sigma$, and

(iii) $t\left(r^{\prime}\right) \in \Sigma$ whenever $r^{\prime}$ is not a prefix of $r$.

We construct an FST $M_{3}=\left(Q_{3}, \bar{\Sigma}, \Delta \cup\{*\}, \delta_{3}, Q_{3, F}\right)$ with

$$
Q_{3}:=\{0,1,2,3\} \times C \times 2^{Q^{3}} \times\left(Q^{3} \cup\{\perp\}\right) \times Q^{3},
$$

where $C$ is the set of subtrees of kernels of proper $\left(z, z^{\prime}\right)$-computations of $M^{3}$,

$$
\begin{aligned}
Q_{2, \mathrm{~F}}:= & \left\{\langle b, \varphi, \varnothing, z, z\rangle \mid b \in\{1,2\}, \varphi \in C \text { is a proper }\left(z, z^{\prime}\right)\right. \text {-computation } \\
& \text { for some } \left.z^{\prime} \in Q^{3}\right\},
\end{aligned}
$$

and

$$
\delta_{3}=\delta^{\prime} \cup \delta^{\prime \prime} .
$$

The set of transitions $\delta^{\prime}$ defines the behavior of $M_{3}$ on the designated path, i.e., for nodes labeled with $*$ or $(a, j)$ for some $a \in \Sigma$, whereas $\delta^{\prime \prime}$ defines the behavior on the remaining nodes, i.e., those labeled by some $a \in \Sigma$. The construction is based on ideas similar to those used for $M_{2}$. Therefore, we omit presenting it explicitly but state the desired properties of $M_{3} . M_{3}$ can be constructed in time $2^{2^{\text {poly(y)/1) }}}$ with $\left|M_{3}\right| \leq 2^{2^{c|M|^{3}}}$ for some constant $c>0$ such that Fact 8 holds.

Fact 8. Assume $t \in T_{\bar{\Sigma}}$ is a tree with a designated branch $r$, and $t^{\prime} \in T_{\Sigma}\left(x_{1}\right)$ is the proper tree obtained from $t$ by replacing the labels $(a, j)$ with $a$ and $*$ with $x_{1}$. Then, for every $s \in T_{\Delta}\left(x_{1}\right)$, the following two statements are equivalent:

(1) $A\langle b, \psi, \varnothing, z, z\rangle$-computation of $M_{2}$ for $t$ exists with $b \in\{1,2\}$ producing output $s[*]$.

(2) Some $\left(z, z^{\prime}\right)$-computation $\varphi$ of $M^{3}$ for $t^{\prime}$ exists such that $\psi=\mathbf{c} \varphi$ and $T_{2}(\varphi)=s$.

Given constructions for $M_{2}$ and $M_{3}$, FST $M_{\gamma}=\left(Q_{\gamma}, \Sigma, \Delta, \delta_{\gamma}, Q_{\gamma, \mathbf{F}}\right)$ is defined as follows (for comments see below):

$$
Q_{\gamma}:=\Gamma_{\gamma} \times Q_{2} \times\left[Q_{3} \cup Q\right],
$$

where $\Gamma_{\gamma}$ is the set of all subtrees of $\gamma$, and the union in the third factor is meant to be disjoint.

$$
Q_{\gamma, \mathrm{F}}:=\left\{\langle\gamma, z, q\rangle \mid z \in Q_{2, \mathrm{~F}}\right\}
$$


provided one of the following conditions hold:

- $\gamma=q$.

- $\gamma=\langle q, h, \tau\rangle\left(\gamma_{1}, \ldots, \gamma_{k}\right)$, and

- $\gamma=\langle q, h, \psi, \tau\rangle\left(\gamma_{1}, \ldots, \gamma_{k}\right)$ and $h \neq \varepsilon$.

It remains to consider specifications $\gamma=\langle q, h, \psi, \tau\rangle\left(\gamma_{1}, \ldots, \gamma_{k}\right)$ where $h=\varepsilon$. If $T\left(M_{\gamma}\right) \neq \varnothing, \psi$ must be a $(\bar{q} \cdot \bar{p})$-computation of $M^{3}$ where $\bar{q}=\left\langle r, q, r^{\prime}\right\rangle$ for some $r, r^{\prime} \in Q$. Then

$$
Q_{\gamma, \mathrm{F}}:=\left\{\left\langle\gamma, z, z^{\prime}\right\rangle \mid z \in Q_{2, \mathrm{~F}}, z^{\prime}=\langle b, \psi, \varnothing, \bar{q}, \bar{q}\rangle \in Q_{3, \mathrm{~F}}\right\} .
$$

$\delta_{\gamma}$ consists of five groups of transitions, namely,

$$
\delta_{\gamma}:=\delta_{y, 1} \cup \cdots \cup \delta_{y, 5},
$$

where $\delta_{\gamma, i}$ are defined as follows:

$\delta_{\gamma, 1}$ consists of all transitions $\left(\left\langle\gamma^{\prime}, z, q\right\rangle, a, s,\left\langle\gamma^{\prime}, z_{1}, q_{1}\right\rangle \cdots\left\langle\gamma^{\prime}, z_{k}, q_{k}\right\rangle\right)$ where

- $\left(z, a, a, z_{1} \cdots z_{k}\right) \in \delta_{2}$,

- $z=\left\langle, h, \ldots,,_{-}\right\rangle$for some $h$ not occurring in $\gamma^{\prime}(\varepsilon)$, and

- $\left(q, a, s, q_{1} \cdots q_{k}\right) \in \delta$.

$\delta_{\gamma, 2}$ consists of all transitions $\left(\left\langle\gamma^{\prime}, z, z^{\prime}\right\rangle, a, s,\left\langle\gamma^{\prime}, z_{1}, z_{1}^{\prime}\right\rangle \cdots\left\langle\gamma^{\prime}, z_{k}, z_{k}^{\prime}\right\rangle\right)$ where

- $\left(z, a, a, z_{1} \cdots z_{k}\right) \in \delta_{2}$, and

- for $z=\left\langle b,,_{-},,_{-}\right\rangle,\left(z^{\prime}, a, s, z_{1}^{\prime} \cdots z_{k}^{\prime}\right) \in \delta_{3}$ provided $b=3$, and $\left(z^{\prime},(a, j), s, z_{1}^{\prime} \cdots z_{k}^{\prime}\right) \in \delta_{3}$ provided $\left(b \in\{0,1,2\}\right.$ and $z_{j}=\left\langle b^{\prime},,_{-,},,_{-}\right\rangle$with $\left.b^{\prime} \in\{0,1,2\}\right)$. $\delta_{\gamma, 3}$ consists of all transitions $\left(\left\langle\gamma^{\prime}, z, z^{\prime}\right\rangle, a, s,\left\langle\gamma^{\prime}, z_{1}, z_{1}^{\prime}\right\rangle \cdots\left\langle\gamma^{\prime}, z_{k}, z_{k}^{\prime}\right\rangle\right)$ where

- $\left(z, a, a, z_{1} \cdots z_{k}\right) \in \delta_{2}$, and

- $\gamma^{\prime}=\langle q, h, \psi, \tau\rangle\left(\gamma_{1}, \ldots, \gamma_{k}\right)$ where $\psi$ is a (proper) $\left(\left\langle p_{-1}, p_{0}, p_{1}\right\rangle,\left\langle p_{-1}^{\prime}, p_{0}^{\prime}, p_{1}^{\prime}\right\rangle\right)$ -

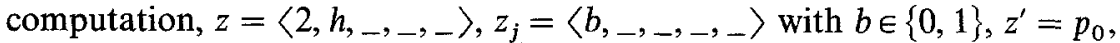
and $\left(\mathrm{z}_{0},(a, j), s, z_{1}^{\prime} \cdots z_{k}^{\prime}\right) \in \delta_{3}$ with $z_{0}=\left\langle b^{\prime}, \psi, \varnothing,\left\langle p_{-1}, p_{0}, p_{1}\right\rangle,\left\langle p_{-1}, p_{0}, p_{1}\right\rangle\right\rangle \quad$ for $\quad b^{\prime} \in\{1,2\}$. $\delta_{\gamma, 4}$ consists of all transitions $\left(\left\langle\gamma^{\prime}, z, q^{\prime}\right\rangle, a, s,\left\langle\gamma_{1}, z_{1}, q_{1}\right\rangle \cdots\left\langle\gamma_{k}, z_{k}, q_{k}\right\rangle\right)$ where

- $\left(z, a, a, z_{1} \cdots z_{k}\right) \in \delta_{2}$,

- $\gamma^{\prime}=\langle q, h, \tau\rangle\left(\gamma_{1}, \ldots, \gamma_{k}\right)$ where $\tau=\left(q^{\prime}, a, s, q_{1} \cdots q_{k}\right)$, and

$\bullet z=\langle 1, h, \ldots, \ldots,-$,$\rangle .$

Finally, $\delta_{\gamma, 5}$ consists of all transitions $\left(\left\langle\gamma^{\prime}, z, z^{\prime}\right\rangle, a, s,\left\langle\gamma_{1}, z_{1}, q_{1}\right\rangle \cdots\left\langle\gamma_{k}, z_{k}, q_{k}\right\rangle\right)$ where

- $\left(z, a, a, z_{1} \cdots z_{k}\right) \in \delta_{2}$,

- $\gamma^{\prime}=\langle q, h, \psi, \tau\rangle\left(\gamma_{1}, \ldots, \gamma_{k}\right)$ where $\psi$ is a $(\bar{p}, \bar{q})$-computation, $\tau=\left(q^{\prime}, a, s\right.$, $\left.q_{1} \cdots q_{k}\right)$, and $q^{\prime}$ is the second component of $\bar{q}$, and

- $z=\left\langle 1, h,,_{-,},\right\rangle$and $z^{\prime}=\left\langle 1, x_{1}, \ldots, \bar{q}, \bar{q}\right\rangle$. 
(As usual, we indicated components of states on which no restriction is imposed by "-.")

$M_{\gamma}$ conceptually runs top-down an input tree $t$. In its first component $M_{\gamma}$ keeps track of the subtree $\gamma^{\prime}$ of $\gamma$ which still has to be checked. In its second component $M_{\gamma}$ simulates $M_{2}$. The computation here is independent of $\gamma$. The third component is used to check a guessed computation of $M$ against $\gamma$. It is this component which produces the output of $M_{\gamma}$.

In detail, the third component operates on a given input tree $t$ with a designated set of nodes $O$ as follows. If $\gamma=q$ the third component of $M_{\gamma}$ simulates $M_{q}$ for $t$. For this, $M_{\gamma}$ uses transitions from group $\delta_{\gamma, 1}$.

Assume $\gamma \notin Q$. Let $\gamma=\langle q, h, \tau\rangle\left(\gamma_{1}, \ldots, \gamma_{k}\right)$ and $t=t_{1} a\left(u_{1}, \ldots, u_{k}\right)$ where $t_{1} / o=x_{1}$ for the node $o \in O_{1}$ of type $h$. Then the third component of $M_{y}$ first simulates a computation of $M_{q}$ for $t_{1}$. This is again done by means of transitions from $\delta_{\gamma, 1}$. At node $o, \tau$ is applied using a transition from $\delta_{\gamma, 4}$. For every subtree $u_{j}, M_{\gamma}$ continues as $M_{\gamma_{j}}$.

Finally, let $\gamma=\langle q, h, \psi, \tau\rangle\left(\gamma_{1}, \ldots, \gamma_{k}\right)$ and $t=t_{1} t_{2} a\left(u_{1}, \ldots, u_{k}\right)$ where $t_{1}, t_{2}$ are proper, $t_{1} / o=x_{1}$, and $t_{1} t_{2} / \operatorname{succ}(o)=x_{1}$ for the (unique) node $o \in O \backslash O_{1}$ of type $h$. Assume $\psi$ is a $\left(\left\langle p_{-1}, p_{0}, p_{1}\right\rangle,\left\langle p_{-1}^{\prime}, p_{0}^{\prime}, p_{1}^{\prime}\right\rangle\right)$-computation of $M^{3}$ and $\tau$ is a $p_{0}^{\prime}$-transition of $M$. Then the third component of $M_{\gamma}$ first simulates a $\left(q, q_{0}\right)$ computation of $M$ for $t_{1}$ using transitions from $\delta_{\gamma_{1},}$. For $t_{2}$ it simulates a $\left(\left\langle p_{-1}, p_{0}, p_{1}\right\rangle,\left\langle p_{-1}^{\prime}, p_{0}^{\prime}, p_{1}^{\prime}\right\rangle\right)$-computation $\varphi$ of $M^{3}$ whose kernel is $\psi$ and produces output $T_{2}(\varphi)$; this is done using, for the third component, states and transitions from $M_{3}$, i.e., by means of transitions from $\delta_{\gamma, 3}$ and $\delta_{\gamma, 2}$. Finally, at node $\operatorname{succ}(o), \tau$ is applied using transitions from $\delta_{\gamma, 5}$, and $M_{\gamma}$ continues with subtrees $u_{j}$ as $M_{y_{j}}$.

Since $M_{\gamma}$ can be constructed in time $2^{2^{\text {poly }(|M|)}}$ with $\left|M_{\gamma}\right| \leq 2^{2^{c \cdot|M|^{3}}}$ for some $c>0$ this completes the proof of Theorem 6.2.

From Theorems 6.1 and 6.2 and their corresponding size versions we conclude the main theorem of this section.

Theorem 6.4. Assume $M$ and $M^{\prime}$ are FSTs.

(i) If $\operatorname{sval}\left(M^{\prime}\right)$ is finite, then it can be decided in space $2^{2^{2 \operatorname{poly} \mid\left(M|+| M^{\prime}\right)}}$ whether or not $\Omega(M) \subseteq \Omega\left(M^{\prime}\right)$.

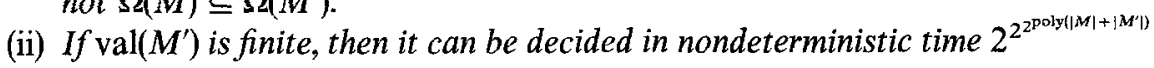
whether $T(M) \nsubseteq T\left(M^{\prime}\right)$.

Proof of (ii). Without loss of generality, assume the input and output signatures of $M$ are disjoint. Then the algorithm is as follows:

(1) Decompose $T\left(M^{\prime}\right)$ into a union $T\left(A_{1}\right) \cup \cdots \cup T\left(A_{K}\right)$ where $K \leq 2^{\left.2^{P\left(M^{\prime}\right)}\right)}$ and $M_{j}$ are single-valued FSTs of size at most $2^{2^{P\left(M M^{\prime}\right)}}$, where $P$ is the polynomial independent of $M$ given by Theorem 6.2.

(2) For $j=1, \ldots, K$, compute an FTA $\bar{M}_{j}$ with $L\left(\bar{M}_{j}\right)=T_{\Sigma} \backslash L\left(M_{j}\right)$.

(3) Guess a subset $I \subseteq\{1, \ldots, K\}$. 
(4) Guess a witness $(t, s) \in T_{\Sigma} \times T_{\Delta}$ and verify that:

- $(t, s) \in T(M)$,

- $\forall j \notin I, t \in L\left(\bar{M}_{j}\right)$, and

- $\forall j \in I, \exists$ accepting computation $\varphi_{j}$ of $M_{j}$ for $t$ such that $s \neq T\left(\varphi_{j}\right)$.

By Theorem 6.2, step (1) can be performed in deterministic time $2^{2^{\text {poly }\left(M M^{\prime}\right)}}$. Step 2 can be implemented by using the standard subset construction. This needs time

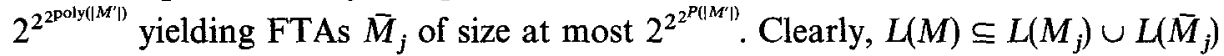
for every $j$. Therefore we can apply Theorem 6.1 to deduce that it suffices in step 4 to guess a witness of depth $2^{2^{\left.2^{P(M(M)}\right)}}$ for some polynomial $P^{\prime}$ in order to let the algorithm accept iff $T(M) \nsubseteq T\left(M^{\prime}\right)$. By using similar ideas as in the algorithm from the proof of Theorem 2.2(ii), the verification part of step (4) can be executed in

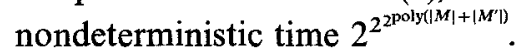

The proof of (i) is analogous where Theorems $6.1 \mathrm{~S}$ and $6.2 \mathrm{~S}$ are used instead of Theorems 6.1 and 6.2 , respectively.

As a corollary of Theorems 5.7 and 6.4 we obtain:

Corollary 6.5. Assume $M$ and $M^{\prime}$ are FSTs.

(i) If $\operatorname{sval}(M)$ is finite, then it can be decided in space $2^{2^{20 \mid \log \left(|M|+\left|M^{\prime}\right|\right)}}$ whether or not $\Omega(M)=\Omega\left(M^{\prime}\right)$.

(ii) If $\operatorname{val}(M)$ is finite, then it can be decided in nondeterministic time $2^{2^{2 \operatorname{poly}\left(M|+| M^{\prime} \mid\right)}}$ whether $T(M) \neq T\left(M^{\prime}\right)$.

\section{Conclusion}

We showed that equivalence is decidable for finite-valued transducers. We presented Properties (F1) and (F2) of an FST $M$ which precisely characterize finite-valuedness of $M$ and derived an upper bound on the depth of a witness $t$ such that $M$ does not have Property $(\mathrm{F} 1)$ or $(\mathrm{F} 2)$ for $t$ provided $\operatorname{val}(M)=\infty$. This allowed us to derive a nondeterministic polynomial-time algorithm to decide whether $M$ is not finite-valued. The question arises whether or not this is the best time complexity that can be hoped for. It turns out that this is not the case. In [Se2] it is shown that, in fact, finite-valuedness of FSTs can be decided in deterministic polynomial time. The polynomial decision procedure is based on a polynomial-time algorithm deciding whether or not an FST is single-valued.

Several questions remain open. The decomposition described in Section 6 decomposes a finite-valued FST $M$ into a possibly double-exponential number of single-valued FSTs - even if the valuedness of $M$ is small (say, 2). In [W5] it is shown that NGSMs of valuedness $k$ in fact can be decomposed into $k$ single-valued NGSMs. It is not clear whether a similar result holds for FSTs as well. Finally, it is also open whether the results of [W3] can be carried over to FSTs, i.e., if it is decidable for FSTs $M$ and $M^{\prime}$ whether or not $T(M)=T\left(M^{\prime}\right)$ provided $\operatorname{sval}\left(M^{\prime}\right)<\infty$. 


\section{Acknowledgment}

The author would like to express his gratitude to one of the referees who contributed to the clarity of the paper by improving the proofs of Propositions 1.1 and 3.2 and insisting on simplifications of the others.

\section{Appendix}

The following basic proposition of Ramsey Theory is taken from [GRS, Chap. 1, Theorem 1]. We sketch a proof of it in order to get an estimation of the numbers involved.

For set $S$ let $[S]^{2}$ denote the set $\left\{\left\{s, s^{\prime}\right\} \subseteq S \mid s \neq s^{\prime}\right\}$ where we simply write $[n]^{2}$ if $S=\{1, \ldots, n\}$. Assume $C$ is a finite set of colors of cardinality $k$. Any map $\rho:[S]^{2} \rightarrow C$ is called $k$-coloring of $[S]^{2}$. A $k$-coloring $\rho$ defines a partition of $[S]^{2}$ into sets $\rho^{-1}(c), c \in C$.

Proposition A. For every $m>2$ and every $k \in \mathbb{N}$, some $n \in \mathbb{N}$ exists such that, for every k-coloring $\rho$ of $[n]^{2},[S]^{2} \subseteq \rho^{-1}(c)$ for some $c \in C$ and $S \subseteq\{1, \ldots, n\}$ of cardinality $m$.

Let $R(m ; k)$ denote the least such number $n \in \mathbb{N}$. Then

$$
R(m ; k) \leq 3 \cdot((m-2) k) !
$$

Proof (Sketch). Without loss of generality, we may assume that, for all involved $k$-colorings, $C=\{1, \ldots, k\}$. For $k \geq 1$ and $a_{j} \geq 2$, define $R\left(a_{1}, \ldots, a_{k}\right)$ as the minimal number $n$ such that, for every $k$-coloring $\rho$ of $[n]^{2},[S]^{2} \subseteq \rho^{-1}(j)$ for some $j \in C$ and $S \subseteq\{1, \ldots, n\}$ of cardinality $a_{j}$.

Clearly, $R(m ; k)=R(m, \ldots, m)$ where $m$ is taken $k$ times. The following facts are easy to prove:

$$
\begin{aligned}
& R\left(a_{1}, \ldots, a_{k}\right)=R\left(a_{\pi(1)}, \ldots, a_{\pi(k)}\right) \quad \text { for every permutation } \pi, \\
& R\left(2, a_{2}, \ldots, a_{k}\right)=R\left(a_{2}, \ldots, a_{k}\right), \\
& R\left(a_{1}, \ldots, a_{k}\right) \leq \sum_{i=1}^{k} R\left(a_{1}, \ldots, a_{i-1}, a_{i}-1, a_{i+1}, \ldots, a_{k}\right) \quad \text { for } k \geq 2, \\
& R\left(a_{1}\right)=a_{1} .
\end{aligned}
$$

We prove, for $k \geq 1$ and $a_{j} \geq 3$,

$$
R\left(a_{1}, \ldots, a_{k}\right) \leq 3 \cdot\left(\sum_{j=1}^{k}\left(a_{j}-2\right)\right) !
$$

The bound in (5) obviously implies the assertion. Estimation (5) is proved by induction on $k$ and, for every $k \geq 2$, by induction on $\sum_{j=1}^{k} a_{j}$. 
Base of induction $(k=1)$. Then

$$
R\left(a_{i}\right)=a_{1} \leq 3 \cdot\left(a_{1}-2\right) !
$$

Induction step $(k \geq 2)$.

$$
\begin{aligned}
R\left(a_{1}, \ldots, a_{k}\right) & \leq \sum_{j=1}^{k} R\left(a_{1}, \ldots, a_{j}-1, \ldots, a_{k}\right) \quad \text { by }(3) \\
& \leq \sum_{j=1}^{k} 3 \cdot\left(\sum_{j=1}^{k}\left(a_{j}-2\right)-1\right) ! \quad \text { by (1) and }(2),
\end{aligned}
$$

where the induction hypothesis for the same $k$ but a smaller total sum of the coefficients is used for those summands where $a_{j}-1>2$, and the induction hypothesis for $k-1$ for the remaining ones

$$
\begin{aligned}
& =k \cdot 3 \cdot\left(\sum_{j=1}^{k}\left(a_{j}-2\right)-1\right) ! \\
& \leq 3 \cdot\left(\sum_{j=1}^{k}\left(a_{j}-2\right)\right) \cdot\left(\sum_{j=1}^{k}\left(a_{j}-2\right)-1\right) ! \text { since } a_{j} \geq 3 \text { for all } j \\
& =3 \cdot\left(\sum_{j=1}^{k}\left(a_{j}-2\right)\right) !
\end{aligned}
$$

\section{References}

[CF] B. Courcelle and P. Franchi-Zannettacchi. Attribute grammars and recursive program schemes, Part I. Theoret. Comput. Sci. 17 (1982), 163-191.

[CK] K. Culik II and J. Karhumäki. The equivalence of finite valued transducers (on HDTOL languages) is decidable. Theoret. Comput. Sci. 47 (1986), 71-84.

[En] J. Engelfriet. Some open questions and recent results on tree transducers and tree languages. In: Formal Language Theory, ed. by R. V. Book, Academic Press, New York, 1980, pp. 241-286.

[Es] Z. Esik. Decidability results concerning tree transducers, I. Acta Cybernet. 5 (1980), 1-20.

[GS1] F. Gecseg and M. Steinby. Tree Automata. Akademiai Kiado, Budapest, 1984.

[GS2] R. Giegerich and K. Schmal. Code selection techniques: Pattern matching, tree parsing and inversion of derivors. Proc. of ESOP, 1988. Lecture Notes in Computer Science, vol. 300, Springer-Verlag, Berlin, pp. 245-268.

[GRS] R. L. Graham, B. L. Rothschild, and J. H. Spencer. Ramsey Theory. Wiley, New York, 1980.

[P] W. Paul. Komplexitätstheorie. Teubner Verlag, Stuttgart, 1978.

[Sc] M. Schützenberger. Sur les relations rationelles entre monoides libres. Theoret. Comput. Sci. 3 (1976), 243-259.

[Se1] H. Seidl. On the finite degree of ambiguity of finite tree automata. Acta Inform. 26 (1989), $527-542$.

[Se2] H. Seidl. Single-valuedness of tree transducers is decidable in polynomial time. Theoret. Comput. Sci. 106 (1992), 135-181.

[W1] A. Weber. Ueber die Mehrdeutigkeit und Wertigkeit von endlichen Automaten und Transducern. Doctoral Thesis, Frankfurt/Main, 1987.

[W2] A. Weber. Decomposing finite-valued transducers and deciding their equivalence. SIAM J. Comput. 22 (1993), 175-202. See also Proc. MFCS, 1988. Lecture Notes in Computer Science, vol. 324, Springer-Verlag, Berlin, pp. 552-562. 
[W3] A. Weber. On the lengths of values in a finite transducer. Acta Inform. 29 (1992), 663-687.

[W4] A. Weber. On the valuedness of finite transducers. Acta Inform. 27 (1990), 749-780.

[W5] A. Weber. Decomposing a $k$-valued tranducer into $k$ unambiguous ones. Proc. LATIN ' 92. Lecture Notes in Computer Science, vol. 583, Springer-Verlag, Berlin, 1992, pp. 503-515.

[WS] A. Weber and H. Seidl, On the degree of ambiguity of finite automata. Theoret. Comput. Sci. 88 (1991), 325-349.

[Z] Z. Zachar. The solvability of the equivalence problem for deterministic frontier-to-root tree transducers. Acta Cybernet. 4 (1978), 167-177.

Received October 9, 1989, and in revised form February 24, 1992, and October 6, 1992. 